\title{
7. NEOGENE DIATOM BIOSTRATIGRAPHY FOR THE EASTERN EQUATORIAL PACIFIC OCEAN, LEG $138^{1}$
}

\author{
Jack G. Baldauf $^{2}$ and Masao Iwai ${ }^{3}$
}

\begin{abstract}
Ocean Drilling Program (ODP) Leg 138 recovered more than $5500 \mathrm{~m}$ of Quaternary to middle Miocene ( 17 Ma) sediments from 11 sites in the eastern equatorial Pacific Ocean. These sediments represent the most complete stratigraphic sequence recovered since the start of scientific ocean drilling by the Deep Sea Drilling Project (DSDP) and ODP. The diatoms observed generally are common to abundant and well-preserved throughout the samples examined. The assemblages are characterized by species typical of low-latitudes and regions of high surface-water productivity and are dominated by Thalassiothrix longissima, Thalassionema nitzschioides, Azpeitia nodulifer, and numerous species of Thalassiosira and Nitzschia.

Fifty-six biostratigraphic events were identified at Sites 844 through 852 , allowing us, in part, to use the diatom zonation of Barron (1985a). This zonation was modified by replacing the Rhizosolenia preabergonii Zone and the upper portion of the Nitzschia jouseae Zone, as used by Barron (1985a), with the Nitzschia marina and Nitzschia jouseae zones, as used by Baldauf (1984, 1987). Twenty-nine biostratigraphic events have been correlated to the Leg 138 paleomagnetic stratigraphy of Schneider (this volume). Nineteen of these events are well constrained to permit recalibration. Diatoms were rare or absent in samples examined from Sites 853 and 854 . As such, these sites are not included in the following discussion.
\end{abstract}

\section{INTRODUCTION}

During Leg 138, 42 holes at 11 sites in the eastern equatorial Pacific Ocean were cored to examine the paleoceanographic history of this region since the middle Miocene. Sites 844 through 847 form a northsouth transect centering at $95^{\circ} \mathrm{W}$ longitude, and Sites 848 through 854 form a north-south transect at $110^{\circ} \mathrm{W}$ longitude (Table 1; Fig. 1). The sediments recovered during this cruise are unique in that they (1) compose near-continuous sequences representing about the last 17 m.y.; (2) have been examined at a sample spacing of 2 to $5 \mathrm{~cm}$ for color spectrum (Mix et al., this volume), GRAPE (Mayer et al., this volume), and magnetic susceptibility (Valet et al., this volume); (3) generally contain the major siliceous and calcareous microfossil groups, and (4) contain at Sites $844,845,848,851$, and 852 , a magnetostratigraphy for portions of the Quaternary through upper middle Miocene sequences. As such, Leg 138 presents a unique opportunity to enhance current biostratigraphies and biochronologies to improve the temporal framework for refined paleoceanographic analysis.

This study presents the diatom biostratigraphic results of samples examined from Sites 844 through 852 . In doing so, calibration of specific stratigraphic markers to magnetostratigraphic records have been reexamined on the basis of comparisons of Leg 138 diatom biostratigraphy and magnetostratigraphy (Schneider, this volume). The sparse occurrence or absence of diatoms from samples examined from Sites 853 and 854 exclude these sites from this study.

\section{METHODS}

\section{Biostratigraphy/Biochronology}

Diatom biostratigraphy for the equatorial Pacific has evolved from the original zonations proposed by Burckle (1972, 1977), Jousé (1973), and Bukry and Foster (1973) to the more recent zonal standard of Barron (1985a). In addition, numerous studies, including

\footnotetext{
${ }^{1}$ Pisias, N.G., Mayer, L.A., Janecek, T.R., Palmer-Julson, A, and van Andel, TH (Eds.), 1995. Proc. ODP, Sci. Results, 138: College Station, TX (Ocean Drilling Program).

2 Department of Oceanography and Ocean Drilling Program, Texas A\&M University, College Station, TX 77845, U.S.A.

${ }^{3}$ Institute of Geology and Paleontology, Tohoku University, Aoba-Ku, Sendai 90 Japan. Present Address: Ocean Research Institute, University of Tokyo, Minamidai,
} Nakano-Ku, Tokyo 164, Japan.
Burckle and Trainer (1979), Burckle et al. (1982), Sancetta (1984), Barron et al. (1985), and Baldauf (1985), have enhanced our understanding of the spatial and temporal distribution of diatoms in the equatorial Pacific region.

The Quaternary and Neogene diatom zonation of Barron (1985a, $1985 \mathrm{~b}$ ) consists of 18 zones and 21 subzones, with each zonal and subzonal boundary defined by the first or last stratigraphic occurrence of a specific species. The majority of these zonal and subzonal biomarkers have been calibrated directly with a magnetostratigraphy based on the studies of Burckle (1972, 1977), Burckle et al. (1982), Barron (1985a, 1985b), and Barron et al. (1985). The zonation used by Barron $(1985 \mathrm{a}, 1985 \mathrm{~b}$ ) results from the studies of eastern equatorial Pacific sediments recovered primarily from DSDP Leg 85. In addition to the primary biostratigraphic markers, numerous secondary stratigraphic markers have also been defined for use in this region. The majority of these secondary events have been indirectly calibrated to the magnetostratigraphy and are considered useful only locally (i.e., the Leg 85/Leg 138 region).

In this study, we adhere to a slightly revised version of the diatom zonation proposed by Barron (1985a, 1985b) for the eastern equatorial Pacific. Barron (1985a) followed Burckle (1977) in using the Rhizosolenia praebergonii Zone as the interval from the first occurrence (FO) of $R$. praebergonii to the FO of Pseudoeunotia doliolus. Within the Leg 138 material, we have followed Baldauf (1984) in replacing, where needed, the $R$. praebergonii Zone with the Nitzschia marina Zone and an extended Nitzschia jouseae Zone (Fig. 2). The $N$. marina Zone is defined as the interval from the FO of $P$. doliolus to the last occurrence (LO) of $N$. jouseae. This zone is subdivided into two subzones on the basis of the LO of Thalassiosira convexa.

Actinocyclus moronensis has a scattered occurrence throughout its stratigraphic range. This limits the usefulness of the LO of $A$. moronensis to define the T. yabei/A. moronensis boundary, following Barron (1985a). We have used the LO of Denticulopsis simonsenii, where necessary, as a secondary marker for this zonal boundary. With the exception of several changes in taxonomic nomenclature discussed by Akiba and Yanagisawa (1985), the remaining zonation used here follows Barron (1985a).

During shipboard analysis, we adhered to the time scale of Berggren et al. (1985); (see Fig. 2). Berggren et al.'s (1985) chronology was slightly modified to incorporate the Blake, Cobb Mountain, and Reunion paleomagnetic events. Estimated ages for the Cobb 


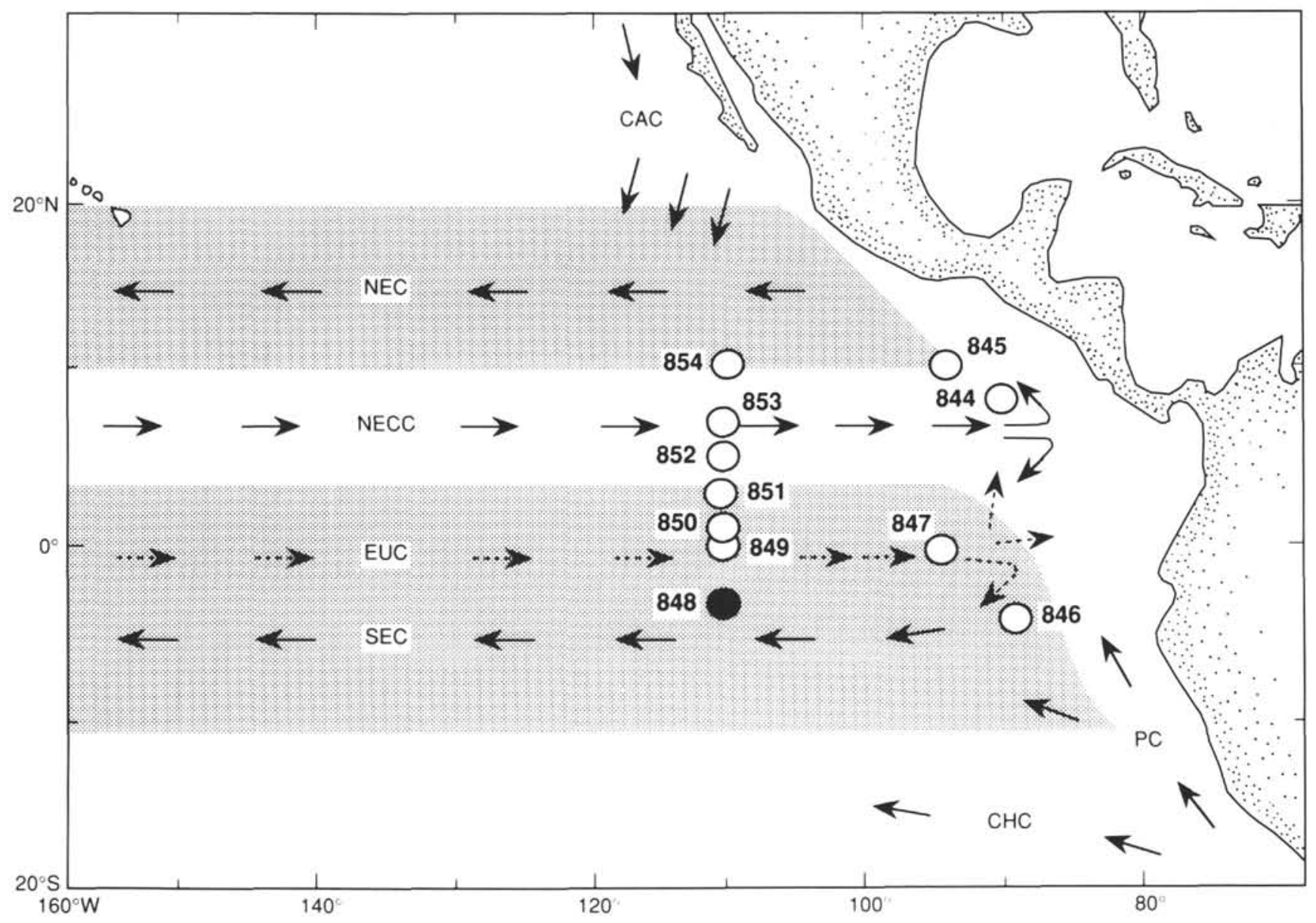

Figure 1. Location of Leg 138 sites (844-854) and generalized circulation system of the eastern equatorial Pacific Ocean. Surface currents = solid arrows, subsurface currents $=$ dashed arrows: $\mathrm{CAC}=$ California Current; $\mathrm{NEC}=$ North Equatorial Current; $\mathrm{NECC}=$ North Equatorial Countercurrent; EUC $=$ Equatorial Undercurrent; $\mathrm{SEC}=$ South Equatorial Current; $\mathrm{PC}=$ Peru Current; and $\mathrm{CHC}=$ Chile Current. Shaded areas represent general latitudinal extent of SEC and NEC

Mountain and Reunion events follow Harland et al. (1982). Calibration of the diatom chronology to the Geopolarity Time Scale (GPTS) of Berggren et al. (1985) follows Barron (1985a, 1985b) and Barron et al. (1985), as Berggren et al. (1985) failed to include calibration of diatoms in their chronostratigraphy. Although for this manuscript we adhered to the more recent chronology of Cande and Kent (1992; see Table 2; Figs. 2 and 3), Berggren et al.'s (1985) chronology also has been presented here to be consistent with shipboard studies.

Biostratigraphic events, such as the first or last occurrences of a specific species, have been assigned to the sample containing the first or last observed specimens. Sample constraints, depth (meters below seafloor), and depth (composite) for each event are presented in the appropriate tables. The composite depth assignment follows that reported in Mayer, Pisias, Janecek, et al. (1992).

\section{Techniques}

Smear slides from selected samples were examined on a routine basis for stratigraphic markers. When required (because of low concentrations of specimens), selected samples from all sites were processed using hydrogen peroxide and hydrochloric acid, following a modified version of the procedures of Baldauf (1984). Strewn slides of the acid-clean material were prepared for samples from Site 845 and from Sites 847 to 852 . These slides were examined using magnifications between 750 and $1250 \times$. Slides examined from Sites $844 \mathrm{~B}$ and $846 \mathrm{~B}$ were generated using a random settling technique similar to that described by Bodén (1991).

\section{RESULTS}

Tables 3 through 5 provide the sample, meters below seafloor depth (mbsf) and meters composite depth (mcd) constraints for stratigraphic events identified for Sites 844 through 846 , located along the eastern transect. Tables 6 through 8 provide similar data for stratigraphic events recognized for Sites 847 through 852 , located along the western transect.

\section{Site 844}

Four holes (844A-844D) were cored at Site 844, situated on the Cocos Plate (Table 1; Fig. 1). Well-preserved diatoms are generally present throughout the Pleistocene (Nitzschia reinholdii Zone) to uppermost lower Miocene (Subzone B of the Crucidenticula nicobarica Zone) sequence. The biostratigraphy presented here for Hole $844 \mathrm{~B}$ has been slightly revised from the shipboard work (see Mayer, Pisias, Janecek, et al., 1992). The continuous occurrence of diatoms combined with an excellent magnetostratigraphy (see Schneider, this volume) for the interval from $\mathrm{C} 1 \mathrm{n}$ through $5 \mathrm{n}(0$ to $\sim 10 \mathrm{Ma})$ make this site an ideal type section for the low-latitude Pacific. In addition, diatom stratigraphic events examined from this site can be directly correlated to those recorded from DSDP Sites 573 through 574 by Barron (1985a).

Samples examined from Core 138-844B-1H were assigned to the Pseusdoeunotia doliolus Zone, based on the occurrence of $P$. doliolus stratigraphically above the LO of Nitzschia reinholdii in Sample 
Table 1. Latitude, longitude, and water depth (drill-pipe measurement) of holes cored during Leg 138.

\begin{tabular}{|c|c|c|c|}
\hline Hole & Latitude & Longitude & $\begin{array}{l}\text { Water } \\
\text { depth } \\
\text { (m) }\end{array}$ \\
\hline $844 \mathrm{~A}$ & $7^{\circ} 55.28 \mathrm{~N}$ & $90^{\circ} 28.85 \mathrm{~W}$ & 3425.0 \\
\hline 844B & $7^{\circ} 55.28 \mathrm{~N}$ & $90^{\circ} 28.85 \mathrm{~W}$ & 3425.0 \\
\hline $844 \mathrm{C}$ & $7^{\circ} 55.28 \mathrm{~N}$ & & \\
\hline 844D & $7^{\circ} 55.28 \mathrm{~N}$ & & \\
\hline $845 \mathrm{~A}$ & $9^{\circ} 34.95 \mathrm{~N}$ & $94^{\circ} 35.45 \mathrm{~W}$ & 3715.9 \\
\hline $845 \mathrm{~B}$ & $9^{\circ} 34.95 \mathrm{~N}$ & $94^{\circ} 35.38 \mathrm{~W}$ & 3715.9 \\
\hline $845 \mathrm{C}$ & $9^{\circ} 34.95 \mathrm{~N}$ & $94^{\circ} 35.38 \mathrm{~W}$ & 3715.9 \\
\hline $846 \mathrm{~A}$ & $3^{\circ} 5.7$ & W & \\
\hline $846 \mathrm{~B}$ & & & 3307.5 \\
\hline $846 \mathrm{C}$ & $3^{\circ} 5.7$ & $90^{\circ} 49.0$ & 3307.5 \\
\hline 846D & $3^{\circ} 5.8$ & $7 \mathrm{~W}$ & 3307.5 \\
\hline $847 \mathrm{~A}$ & $0^{\circ} 11.5$ & $\mathrm{w}$ & \\
\hline $847 \mathrm{~B}$ & & & \\
\hline 847 & 0 & $95^{\circ} 1$ & 3346.0 \\
\hline 847D & 0 & $95^{\circ} 19.2$ & 3346 \\
\hline $848 \mathrm{~A}$ & & $110^{\circ} 28.7$ & \\
\hline 848B & & & \\
\hline & $2^{\circ} 59$. & & \\
\hline 8481 & is & W & 3866.1 \\
\hline 849 & & & 384 \\
\hline & & & \\
\hline & & & \\
\hline & $0^{\circ} 10$ & & \\
\hline 850 & & & 3797 \\
\hline & & & \\
\hline & & & \\
\hline & & & \\
\hline 851 & $2^{\circ} 46$. & & 3772.0 \\
\hline & & & \\
\hline & & & \\
\hline & & $110^{\circ}$ & \\
\hline & & & \\
\hline & & & \\
\hline & & & \\
\hline & $7^{\circ} 12$ & $109^{\circ} 45.0$ & \\
\hline & & & \\
\hline & $7^{\circ} 12$ & & \\
\hline & & & \\
\hline & & $099^{4} 5$. & \\
\hline 854 & $11^{\circ} 13$ & $09^{\circ} 35.6$ & \\
\hline $854 \mathrm{E}$ & & & \\
\hline $854 \mathrm{C}$ & $11^{\circ} 13.43 \mathrm{~N}$ & $109^{\circ} 35.65 \mathrm{~W}$ & 3579 . \\
\hline
\end{tabular}

138-844B-2H-CC. The interval from the LO of $N$. reinholdii in Sample 138-844B-2H-CC and the FO of $P$. doliolus in Sample 138$844 \mathrm{~B}-3 \mathrm{H}-2,60 \mathrm{~cm}$, was assigned to the $N$. reinholdii Zone (Table 3 ). The scattered occurrence of $R$. praebergonii at this site did not allow us to place the $N$. reinholdii $\mathrm{A} / \mathrm{B}$ subzonal boundary.

The interval from Samples 138-844B-3H-4, $60 \mathrm{~cm}$, through - $4 \mathrm{H}-3$, $60 \mathrm{~cm}$, contain specimens of Nitzschia marina without specimens of $P$. doliolus or Nitzschia jouseae. This interval was assigned to the $N$. marina Zone, as used by Baldauf (1984). The LO of Thalassiosira convexa, which marks the Subzone A/B boundary, was not recognized in Hole 844B, but has been placed in Sample 138-844C-3H-6, $35 \mathrm{~cm}$. Specimens of $R$. praebergonii occur sporadically throughout this interval in both holes. In Hole $844 \mathrm{~B}$, the lowest stratigraphic occurrence of this species is seen in Sample 138-844B-4H-3, 60 cm, which approximates the LO of $N$. jouseae. This stratigraphic placement suggests that this does not reflect the true LO of this species.

The stratigraphic sequence representing the lower portion of the lower Pliocene $N$. jouseae Zone through the upper Miocene Actinocyclus moronensis Zone (representing about a $5 \mathrm{~m}$.y. interval) is condensed and occurs in the interval from the lower portion of Core $138-844 \mathrm{~B}-4 \mathrm{H}$ through Section 138-844B-8H-2. Biostratigraphic events recognized in this interval include the FO of $T$. convexa in Sample 138-844B-5H-1, $120 \mathrm{~cm}$; FO of Thalassiosira praeconvexa in Sample 138-844B-5H-2, $120 \mathrm{~cm}$; FO of Nitzschia miocenica in Sample 138-844B-5H-3, $120 \mathrm{~cm}$; LO of Thalassiosira yabei and the FO of Nitzschia cylindrica in Sample 138-844B-6H-1, $120 \mathrm{~cm}$; FO of
Table 2. Age estimates for primary and secondary diatom biostratigraphic events for the equatorial Pacific Ocean.

\begin{tabular}{|c|c|c|c|}
\hline Diatom event & Calibration/reference & $\begin{array}{l}\text { Age } \\
(* 1)\end{array}$ & $\begin{array}{l}\text { Age } \\
(* 2)\end{array}$ \\
\hline T. Nitzschia reinholdii & CIn (Burckle, 1977) & 0.65 & 0.65 \\
\hline T. Nitzschia fossilis & & 0.85 & 0.92 \\
\hline T. Rhizosolenia matuvama & CIn.In (Burckle, 1978) & 0.94 & 1.01 \\
\hline B. Rhizosolenia matuyama & & 1.10 & 1.17 \\
\hline T. Rhizosolenia praebergonii var. robusta & Cln (Burckle \& Trainer. 1979) & 1.55 & 1.64 \\
\hline B. Pseudoeunotia doliolus & C2n (Burckle, 1977) & 1.80 & 1.90 \\
\hline T. Rhizosolenia praebergonii & $\mathrm{C} 2 \mathrm{n}$ (Burckle, 1978) & 1.85 & 1.95 \\
\hline T. Thalassiosira convexa & C2n (Burckle, 1978) & 2.10 & 2.21 \\
\hline T. Nitzschia jouseae & C2An.In (Burckle, 1978) & 2.60 & 2.73 \\
\hline B. Rhizosolenia praehergonii & C2An.2n (Burckle. 1978) & 3.00 & 3.14 \\
\hline B. Thalassiosira convexa var. convexa & C2An (Burckle \& Trainer, 1979) & 3.60 & 3.75 \\
\hline B. Asteromphalus elegans & C.3n. In ( Burckle, 1978) & 3.90 & 4.06 \\
\hline T. Nit:schia crlindrica & C.3n.3n ( Burckle. 1978) & 4.30 & 4.50 \\
\hline B. Nitzschia jouseae & C3n.3n (Burckle, 1978) & 4.50 & 4.73 \\
\hline B. Thalassiosira oestrupii & & 5.10 & 5.42 \\
\hline T. Thalassiosira miocenica & (Burckle, 1978) & 5.10 & 5.42 \\
\hline T. Nitzschia miocenica & C3An.In (Burckle, 1972) & 5.55 & 5.96 \\
\hline T. Nitzschia miocenica var. elongata & C3An.In (Burckle. 1978) & 5.65 & 6.05 \\
\hline T. Thalassiosira praeconvexa & C.3An.2n (Burckle, 1978) & 5.80 & 6.25 \\
\hline B. Thalassiosira miocenica & C3An.2n (Burckle, 1978) & 6.10 & 6.54 \\
\hline B. Thalassiosira convexa & C3An.2n (Burckle, 1978) & 6.10 & 6.54 \\
\hline B. Thalassiosira praeconvexa & C3An.2n (Burckle, 1978) & 6.30 & 6.69 \\
\hline T. Nitzschia porteri & C4n.In (Burckle, 1978) & 6.70 & 7.25 \\
\hline B. Nitzschia miocenica & & 6.75 & 7.33 \\
\hline T. Rossiella paleacea & C4n.In (Burckle, 1978) & 6.80 & 7.40 \\
\hline T. Thalassiosira burckliana & C4n.2n (Burckle, 1978) & 7.00 & 7.61 \\
\hline B. Nitschia reinholdii & & 7.30 & 7.94 \\
\hline B. Nitzschia marina & & 7.40 & 8.07 \\
\hline T. Thalassiosira yabei & C4n.In (Burckle, 1978) & 7.55 & 8.21 \\
\hline B. Nitzschia cylindrica & & 7.55 & 8.21 \\
\hline T. Coscinodiscus loeblichii & & 7.90 & 8.53 \\
\hline B. Nitzschia fossilis & & 8.10 & 8.74 \\
\hline B. Thalassiosira burckliana & C4An (Burckle. 1978) & 8.20 & 8.85 \\
\hline B. Coscinodiscus loeblichii & & 8.40 & 9.06 \\
\hline T. Azpeitia vetustissimus var. javanicus & & 8.55 & 9.22 \\
\hline B. Azpeitia vetustissimus var, javanicus & & 8.75 & 9.46 \\
\hline T. Denticulopsis simonsenii & & 8.80 & 9.49 \\
\hline T. Actinocyclus moronensis & C4An.2n (Burckle, 1978) & 8.90 & 9.58 \\
\hline B. Actinocyclus ellipticus var. lanceolata & & 9.90 & 10.40 \\
\hline T. Synedra jouseama & & 10.40 & 10.82 \\
\hline B. Rossiella paleacea var. elongata & & 10.60 & 11.00 \\
\hline T. Crasepedodiscus cosinodiscus & & 10.60 & 11.00 \\
\hline T. Coscinodiscus gigas var. diorama & & 10.70 & 11.09 \\
\hline T. Actinocyclus ellipticus var, spiralis & & 10.80 & 11.17 \\
\hline B. Hemidiscus cuneiformis & C5n.2n (Burckle. 1978) & 11.10 & 11.44 \\
\hline B. Rossiella praepaleacea & & 11.45 & 11.81 \\
\hline T. Actinocyclus ingens & & 11.50 & 11.81 \\
\hline T. Cestodiscus pulchellus & & 11.60 & 11.89 \\
\hline B. Nitzschia porteri & & 11.70 & 11.98 \\
\hline B. Coscinodiscus temperi var, delicata & & 11.80 & 12.06 \\
\hline T. Crucidenticula nicobarica & & 12.20 & 12.40 \\
\hline T. Annellus californicus & & 12.40 & 12.57 \\
\hline B. Coscinodiscus gigas & & 12.60 & 12.74 \\
\hline T. Coscinodiscus lewisianus & & 12.80 & 12.92 \\
\hline B. Denticulopsis panctata & & 13.00 & 13.09 \\
\hline T. Thalassiosira tappanae & & 13.25 & 13.30 \\
\hline B. Azpeitia nodulifer & & 13.30 & 13.34 \\
\hline B. Triceratium cinnamomeum & & 13.40 & 13.43 \\
\hline B. Denticulopsis simonsenii & & 13.65 & 13,64 \\
\hline B. Actinocyclus ellipticus var. spiralis & & 14.20 & 14.16 \\
\hline T. Cestodiscus peplum & & 14.20 & 14.16 \\
\hline B. Actinocyclus ellipticus & & 14.40 & 14.36 \\
\hline B. Coscinodiscus blysmos & & 14.60 & 14.55 \\
\hline B. Thalassiosira tappanae & & 14.60 & 14.55 \\
\hline T. Annellus californicus & & 15.00 & 14.92 \\
\hline T. Azpeitia praenodulifer & & 15.40 & 15.28 \\
\hline B. Actinocyclus ingens & & 15.50 & 15.37 \\
\hline T. Nitzschia maleinterpretaria & & 15.60 & 15.47 \\
\hline T. Coscinodiscus lewisiamus var. simillis & & 15.70 & 15.56 \\
\hline T. Crucidenticula kanayae & & 16.00 & 15.83 \\
\hline T. Thalassiosira fraga & & 16.20 & 16.02 \\
\hline B. Cestodiscus peplum & & 16.40 & 16.20 \\
\hline T. Raphidodiscus marylandicus & & 16.75 & 16.53 \\
\hline B. Crucidenticula kanayae & & 16.90 & 16.68 \\
\hline
\end{tabular}

Note: $\mathrm{T}=$ top or last occurrence; $\mathrm{B}=$ bottom or first occurrence; $\mathrm{I} \mathrm{l}=$ ages using Berggren et al. (1985): 2 = ages using Cande and Kent (1992). 
Table 3. Sample constraints of stratigraphically useful events from eastern transect Holes 844B through 847D.

\begin{tabular}{|c|c|c|c|c|c|c|c|c|c|c|}
\hline Event & $\begin{array}{l}\text { Hole } \\
844 \mathrm{~B}\end{array}$ & $\begin{array}{l}\text { Hole } \\
844 \mathrm{C}\end{array}$ & $\begin{array}{l}\text { Hole } \\
845 \mathrm{~A}\end{array}$ & $\begin{array}{l}\text { Hole } \\
845 \mathrm{~B}\end{array}$ & $\begin{array}{l}\text { Hole } \\
846 \mathrm{~B}\end{array}$ & $\begin{array}{l}\text { Hole } \\
846 \mathrm{C}\end{array}$ & $\begin{array}{l}\text { Hole } \\
846 \mathrm{D}\end{array}$ & $\begin{array}{l}\text { Hole } \\
847 \mathrm{~B}\end{array}$ & $\begin{array}{l}\text { Hole } \\
847 \mathrm{C}\end{array}$ & $\begin{array}{l}\text { Hole } \\
847 \mathrm{D}\end{array}$ \\
\hline T. N. reimholdii & $1-\mathrm{CC} / 2 \mathrm{CC}$ & $<1-\mathrm{CC}$ & $3-5,120 / 3-6,120$ & $1-\mathrm{CC} / 2-\mathrm{CC}$ & $3-3,70 / 3-3,121$ & $2-\mathrm{CC} / 3-\mathrm{CC}$ & & $1-\mathrm{CC} / 2-6,70$ & $1-\mathrm{CC} 2-\mathrm{CC}$ & $1-\mathrm{CC} / 2-\mathrm{CC}$ \\
\hline $\begin{array}{l}\text { 1. N. reminhotal } \\
\text { T. N. fossilis }\end{array}$ & $1=\mathrm{CC} / \mathrm{CL}$ & $1-1,70 / 1-2,40$ & 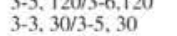 & $1-\mathrm{Cl} / 2 \mathrm{-C}$ & $\begin{array}{l}3-3.40 / 3-\mathrm{CC} \\
3-1,1\end{array}$ & $2-\mathrm{CC} / 3-\mathrm{CC}$ & $2-\mathrm{CC} / 3-\mathrm{CC}$ & $2-\mathrm{CC} / 3-3,70$ & $2-\mathrm{CC} / 3-\mathrm{CC}$ & 2-CC/3-CC \\
\hline T. $R$ matuyama & & & $3-5,30 / 3-6,30$ & & $4-6.75 / 4-\mathrm{CC}$ & $3-\mathrm{CC} / 4-\mathrm{CC}$ & $3-\mathrm{CC} / 4-\mathrm{CC}$ & & & \\
\hline & & & $3-6.30 / 3-7,30$ & & $5-4.75 / 5-\mathrm{CC}$ & 4. CC/5-CC & 4-CC5-CC & $6-1,60 / 6-4,60$ & $5-6.2015-\mathrm{CC}$ & 5-CC/6-CC \\
\hline $\begin{array}{l}\text { T. R. praebergoni v. robustus } \\
\text { B. P. doliolus }\end{array}$ & $3-2,60 / 3-4,60$ & 1.CC/2-4.35 & $4-C C / 5-1.30$ & $3-\mathrm{CC} / 4-\mathrm{CC}$ & $\begin{array}{l}7-3,120 / 7-4,20 \\
7-\mathrm{CC} / 8-1,125\end{array}$ & $\begin{array}{l}\text { 6-CC/7-CC } \\
7-\mathrm{CC} / 8-\mathrm{CC}\end{array}$ & $\begin{array}{l}\text { 5-CC/6-CC } \\
7-5.107-5.80\end{array}$ & $\begin{array}{l}6.1 .60 / 6-4.60 \\
7.5,60 / 7-6.60\end{array}$ & $\begin{array}{l}5-6.2015-\mathrm{CCC} \\
6-\mathrm{CC} 7-\mathrm{CC}\end{array}$ & $\begin{array}{l}\mathrm{B}-\mathrm{CC} 6-\mathrm{CL} \\
6-\mathrm{CC} 7 \cdot \mathrm{CC}\end{array}$ \\
\hline T. $T$. comvexas s. & & $3-3,35 / 3-6,35$ & $5-5.120 / 5-6.120$ & & $8-4,12018-5,120$ & $9-\mathrm{CC} / 10-\mathrm{CC}$ & $8-2.130 / 8-3.10$ & $8-6.60 / 8-\mathrm{CC}$ & 7-CC/8-CC & 7-CC/8-CC \\
\hline T. N. jouseae & $4-3,60 / 4-\mathrm{CC}$ & & $6-1,120 / 6-2,120$ & $4 \mathrm{CC} / 5-\mathrm{CC}$ & $|1-1,110 / 11-2,12|$ & $10-\mathrm{CC} / 1-\mathrm{CC}$ & $11-1.60 / 11-1,120$ & 9. $\mathrm{CC} / 10-2,60$ & $9-\mathrm{CC} / 10-\mathrm{CC}$ & $9-\mathrm{CC} / 10-4,120$ \\
\hline $\begin{array}{l}\text { B. R. pracbergonii s.l. } \\
\text { F. T. comexa v. comvexa } \\
\text { B. comvers }\end{array}$ & $4-3,60 / 4-\mathrm{CC}$ & $2-\mathrm{CC} / 3-\mathrm{CC}$ & $\begin{array}{l}6-2.120 / 6-3,120 \\
7-1.120 / 7-2,120\end{array}$ & $5-\mathrm{CC} / 6-\mathrm{CC}$ & $\begin{array}{l}12-6.120 / 12-6,140 \\
14-C C / 15-1,148\end{array}$ & $10-\mathrm{CC} / 12-\mathrm{CC}$ & $\begin{array}{l}11-\mathrm{CC} / 12-\mathrm{CC} \\
14-6,10 / 14-6,100\end{array}$ & $\begin{array}{l}10-\mathrm{CC} / 11 \mathrm{-CC} \\
12-5.70 / 2 \mathrm{CC}\end{array}$ & $\begin{array}{l}\text { 10-2, 148/10-4, } 148 \\
11-\mathrm{CC} / 12-\mathrm{CC}\end{array}$ & $\begin{array}{l}\text { 10-CC/11-CC } \\
11-\mathrm{CC} / 12-\mathrm{CC}\end{array}$ \\
\hline $\begin{array}{l}\text { B. A. elegans } \\
\text { T. } N \text { cclindrica }\end{array}$ & & & $8-2.27 / 8-3,27$ & & 18-CC/19-1,148 & 17-CC/18-CC & $18 \mathrm{X}-\mathrm{CC} / 20 \mathrm{X}-\mathrm{CC}$ & 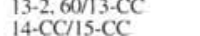 & $15 \mathrm{X}-6,75 / 15 \mathrm{X}-\mathrm{CC}$ & \\
\hline $\begin{array}{l}\text { B... jousece } \\
\text { B.T. oestrupii }\end{array}$ & $4-\mathrm{CC} / 5-1,120$ & & $\begin{array}{l}8-1.27 / 8-2,27 \\
8-6.120 / 8 .-C C\end{array}$ & 7-CC/8-CC & 19-CC/20-1, 148 & $18-\mathrm{CC} / 19-\mathrm{CC}$ & $19-5,120 / 19-6,30$ & $18 X-2,90 / 18 X-4,90$ & $17 \mathrm{X}-\mathrm{CC} / 18 \mathrm{X}-3,120$ & \\
\hline $\begin{array}{l}\text { B.T. os struppii } \\
\text { T. T. miscenica }\end{array}$ & & & $\begin{array}{l}8.0 .120 / 8-4 C, 120 \\
9-3,120 / 9-4\end{array}$ & & $22-4,148 / 22-5,80$ & & $21 \mathrm{X}-\mathrm{CC} / 22 \mathrm{X}-\mathrm{CC}$ & 19X-CC/20X-5, 60 & $20 X-1,12020 x-2,120$ & \\
\hline T. N. mincenica & 4-CC/5-1. 120 & $4-3.75 / 4.4,75$ & $9-5,120 / 9-6,120$ & & $23 \mathrm{X}-7,40 / 23 \mathrm{X}-\mathrm{CC}$ & & $22 \mathrm{X}-\mathrm{CC} / 23 \mathrm{X}-\mathrm{CC}$ & & & \\
\hline T. N. miocenica v, elongata & $\begin{array}{l}4-\mathrm{CC} / 5-1,120 \\
4 \mathrm{CC} / 5-1,20\end{array}$ & $4-1.75 / 4-3,75$ & & & $\begin{array}{l}23 \mathrm{X}-\mathrm{CC} / 24 \mathrm{X}-\mathrm{CC} \\
33 \mathrm{X}-\mathrm{CC} 24 \mathrm{X}-\mathrm{CC}\end{array}$ & & $22 \mathrm{x}-\mathrm{CCO} 3 \mathrm{x}-\mathrm{CC}$ & $22 x-C C 23 x-1-60$ & $21 \times-3.100 / 21 \times-5=50$ & \\
\hline $\begin{array}{l}\text { 1. . proecconvexa } \\
\text { B. T. miecenica }\end{array}$ & $4-\mathrm{CC} / 5-1.120$ & & 9-CC/10-CC & & $25 \mathrm{X}-5,1 / 25 \mathrm{X}-\mathrm{CC}$ & & $24 \mathrm{X}-\mathrm{CC} / 25 \mathrm{X} \cdot \mathrm{CC}$ & & & \\
\hline B. T. convexa v. aspinosa & $5-1,120 / 5-2,120$ & $4-3,75 / 4-4,75$ & 9-CC/10-CC & & $25 \mathrm{X}-5,1 / 25 \mathrm{X}-\mathrm{CC}$ & & $24 \mathrm{X}-\mathrm{CC} / 25 \mathrm{X}-\mathrm{CC}$ & & & \\
\hline B. T. preccomvexa & $5-2,120 / 5-3,120$ & & & & $25 \mathrm{X}-\mathrm{CC} / 26 \mathrm{X}-1.120$ & & $25 \mathrm{X}-\mathrm{CC} / 26 \mathrm{X}-\mathrm{CC}$ & & & \\
\hline $\begin{array}{l}\text { T. N. porteri } \\
B . N . \text { miocenica }\end{array}$ & $5-3,12015-5,120$ & 4-CC/5-CC & $11-1,29 / 11-3,120$ & & $\begin{array}{l}27 X-2,120 / 27 X-3,75 \\
27 X-6,50 / 27 X-C C\end{array}$ & & & & & \\
\hline $\begin{array}{l}\text { B. N. paleacea } \\
\text { T. } R \text {. paleace }\end{array}$ & $5-3,12015-5,120$ & 4. $\mathrm{CC} / 5-\mathrm{CC}$ & & & $28 \mathrm{X}-3,60 / 28 \mathrm{X}-3,80$ & & & & & \\
\hline B. N. reinholdii & $5-5,12015-6,120$ & 4-CC/5-CC & & $11 \mathrm{CC} 13.489$ & & & & & & \\
\hline $\begin{array}{l}\text { T. T. yabei Group } \\
\text { B. N. cylindrica }\end{array}$ & $\begin{array}{l}5-\mathrm{CC} / 6-1,120 \\
6-1,120 / 6-2,120\end{array}$ & $5 \cdot \mathrm{CC} / 6-\mathrm{CC}$ & $12-6.30 / 12-\mathrm{CC}$ & $11-\mathrm{CC} / 13-4,89$ & $\begin{array}{l}30 \times-1.100 / 30 \times-1,122 \\
30 \times-1.60 / 30 \times-1.122\end{array}$ & & & & & \\
\hline $\begin{array}{l}\text { T. C. loeblichii } \\
\text { B. Thurckliang }\end{array}$ & $\begin{array}{l}6-4,6016-C \mathrm{CC} \\
6-4,60 / 6-\mathrm{CC}\end{array}$ & & & & & & & & & \\
\hline B. C. loeblichii & 6-CC 7 -CC & & & & & & & & & \\
\hline T. D. simonsenii s.I. & $7-5.6017-\mathrm{CC}$ & 6-CC/7-CC & $14-1.120 / 14-C C$ & $13-4.89 / 16-\mathrm{CC}$ & $31 \mathrm{X}-\mathrm{CC} / 32 \mathrm{X}-1,60$ & & & & & \\
\hline $\begin{array}{l}\text { T. A. moronensis } \\
\text { T. A. ellipticus f. lanceolata }\end{array}$ & $8-1.60 / 8-2,60$ & 6-CC $77-\mathrm{CC}$ & & & $32 \mathrm{X}-\mathrm{CC} 233 \mathrm{X}-\mathrm{CC}$ & & & & & \\
\hline $\begin{array}{l}\text { T. A. ellipticus f. lanceolata } \\
\text { T. S. jouseana }\end{array}$ & 9-CCr10-CC & & & & $32 \mathrm{X}-\mathrm{CC} / 33 \mathrm{X}-\mathrm{CC}$ & & & & & \\
\hline $\begin{array}{l}\text { T. C, coscinodiscus } \\
\text { B. R.paleacea y elonguta }\end{array}$ & $\begin{array}{l}\text { 9-CC/10-CC } \\
9-\mathrm{CC} / 0-\mathrm{CC}\end{array}$ & $8-\mathrm{CC} 99-\mathrm{CC}$ & & $15-\mathrm{CC} / 16-\mathrm{CC}$ & $\begin{array}{l}34 X-6,90 / 34 X-6,120 \\
34 \times-6,90 / 34 X-6,120\end{array}$ & & & & & \\
\hline $\begin{array}{l}\text { T. } . \text { gigas v, diorama } \\
\text { T. }\end{array}$ & & & 16-CC/17-CC & & $34 \mathrm{X}-6,90 / 34 \mathrm{X}-6,120$ & & & & & \\
\hline $\begin{array}{l}\text { T. A. ingens } \\
\text { T. C pulchellus }\end{array}$ & 12-CC/13-CC & $12-\mathrm{CC} / 13-\mathrm{CC}$ & 18-CC/19-1, 120 & $18-\mathrm{CC} / 19-3,20$ & $36 \mathrm{X}-4,30 / 36 \mathrm{X}-5,103$ & & & & & \\
\hline $\begin{array}{l}\text { T. C. pulchellus } \\
\text { B. N. porteri }\end{array}$ & 12-CC/13-CC & & 17-CC/18-CC & & $36 \mathrm{X}-5,103 / 36 \mathrm{X}-\mathrm{CC}$ & & & & & \\
\hline $\begin{array}{l}\text { T. C. temperi v. delicata } \\
\text { T.A californicus }\end{array}$ & 12-CC/13-CC & $12 \mathrm{CCC1}_{3} \mathrm{CC}$ & & & $36 \mathrm{X}-5,103 / 36 \mathrm{X}-5,120$ & & & & & \\
\hline $\begin{array}{l}\text { T. A. califormicus } \\
\text { B.C. gigas v. diorama }\end{array}$ & $12-\mathrm{CC} / 13-\mathrm{CC}$ & 12-CC/13-CC & & & $38 \mathrm{X}-2,90 / 38 \mathrm{X}-3,30$ & & & & & \\
\hline $\begin{array}{l}\text { D. C. lewisianus } \\
\text { T. C. loma }\end{array}$ & $\begin{array}{l}\text { 14-CC/15-CC } \\
15-\mathrm{CC} / 16-\mathrm{CC}\end{array}$ & $14-\mathrm{CC} / 15-\mathrm{CC}$ & $20-3,120 / 20-\mathrm{CC}$ & 19- $\mathrm{CC} / 20-3.67$ & $38 \mathrm{X}-1,90 / 38 \mathrm{X}-3,120$ & & & & & \\
\hline B. T. cinnamomeum & $15-\mathrm{CC} / 16-\mathrm{CC}$ & $15-\mathrm{CC} / 16-\mathrm{CC}$ & & & & & & & & \\
\hline B. D. simonsenii s.I. & 16-CC/17-CC & & & & & & & & & \\
\hline T. C. peplum & 17-CC/18-CC & & $24 \mathrm{X}-\mathrm{CC} / 26 \mathrm{X}-\mathrm{CC}$ & & & & & & & \\
\hline $\begin{array}{l}\text { B. A. ellipticus } \\
\text { B. C blsmos }\end{array}$ & $\begin{array}{l}\text { 18-CCI19-CC } \\
19-\mathrm{CC} / 20-\mathrm{CC}\end{array}$ & & & & & & & & & \\
\hline $\begin{array}{l}\text { B. C. blysmos } \\
\text { T. A. californicus }\end{array}$ & $\begin{array}{l}\text { 19-CC/20-CC } \\
21 \mathrm{X}-\mathrm{CC} / 22 \mathrm{X}-\mathrm{CC}\end{array}$ & & & & & & & & & \\
\hline $\begin{array}{l}\text { 1. A. califormicus } \\
\text { B. A. ingens }\end{array}$ & $23 \mathrm{X}-\mathrm{CC} / 24 \mathrm{X}-\mathrm{CC}$ & & & & & & & & & \\
\hline $\begin{array}{l}\text { T. C. lewisiamus v. simillis } \\
\text { T. T. fraga }\end{array}$ & $\begin{array}{l}24 \mathrm{X}-\mathrm{CC} / 25 \mathrm{X}-\mathrm{CC} \\
28 \mathrm{CC} 0 \mathrm{x}-7.40\end{array}$ & & & & & & & & & \\
\hline B. C. pephum & $28 \mathrm{X}-\mathrm{CC} / 29 \mathrm{X}-7,40$ & & $29 \mathrm{X}-\mathrm{CC} / 30 \mathrm{X}-\mathrm{CC}$ & & & & & & & \\
\hline T. R. marylandicus & $28 \mathrm{X}-\mathrm{CC} / 29 \mathrm{X}-7,40$ & & & & & & & & & \\
\hline
\end{tabular}

Note: $\mathrm{T}=$ top or last occurrence of a species; $\mathrm{B}=$ bottom or first occurrence of a species; $\mathrm{CC}=$ core catcher; $\mathrm{X}=$ extended core barrel core. All samples were taken from APC-cored material unless noted otherwise. 
Table 4. Depth constraints of stratigraphically useful events from eastern transect Holes 844B through 847D.

\begin{tabular}{|c|c|c|c|c|c|c|c|c|c|c|c|c|c|c|c|c|c|c|c|c|}
\hline $\begin{array}{l}\text { Event } \\
(\mathrm{mbs})\end{array}$ & $\begin{array}{c}844 \mathrm{~B} \\
\text { top }\end{array}$ & $\begin{array}{r}844 \mathrm{~B} \\
\text { bottom }\end{array}$ & $\begin{array}{l}844 \mathrm{C} \\
\text { top }\end{array}$ & $\begin{array}{l}844 \mathrm{C} \\
\text { bottom }\end{array}$ & $\begin{array}{c}845 \mathrm{~A} \\
\text { top }\end{array}$ & $\begin{array}{l}845 \mathrm{~A} \\
\text { bottom }\end{array}$ & $\begin{array}{l}845 B \\
\text { top }\end{array}$ & $\begin{array}{l}845 \mathrm{~B} \\
\text { bottom }\end{array}$ & $\begin{array}{l}846 \mathrm{~B} \\
\text { top }\end{array}$ & $\begin{array}{l}846 \mathrm{~B} \\
\text { bottom }\end{array}$ & $\begin{array}{l}846 \mathrm{C} \\
\text { top }\end{array}$ & $\begin{array}{l}846 \mathrm{C} \\
\text { bottom }\end{array}$ & $\begin{array}{l}846 \mathrm{D} \\
\text { top }\end{array}$ & $\begin{array}{l}846 \mathrm{D} \\
\text { botiom }\end{array}$ & $\begin{array}{l}847 \mathrm{~B} \\
\text { top }\end{array}$ & $\begin{array}{l}847 \mathrm{~B} \\
\text { botrom }\end{array}$ & $\begin{array}{l}847 \mathrm{C} \\
\text { top }\end{array}$ & $\begin{array}{l}847 \mathrm{C} \\
\text { bottom }\end{array}$ & $\begin{array}{l}847 \mathrm{D} \\
\text { top }\end{array}$ & $\begin{array}{l}847 \mathrm{D} \\
\text { bottom }\end{array}$ \\
\hline T. N. reinholdii & 4.53 & 14,48 & & & 24.3 & 25.8 & 9.73 & 21.49 & 18.7 & 21.7 & 21.84 & 31.45 & & & 6.59 & 14.7 & 11.97 & 21.34 & $6.6 !$ & 16.54 \\
\hline T. N. fassils & & & 0.7 & 1.9 & 20.4 & $\begin{array}{l}23.4 \\
24.9\end{array}$ & & & 21.7 & $\begin{array}{l}26.17 \\
36.06\end{array}$ & $\begin{array}{l}21.84 \\
31.45\end{array}$ & $\begin{array}{l}31.45 \\
41.09\end{array}$ & $\begin{array}{l}21.61 \\
32.03\end{array}$ & 32.93 & 16.08 & 19.7 & 21.34 & 31.07 & 16.54 & 25.84 \\
\hline $\begin{array}{l}\text { T. R. matuyama } \\
\text { B. } R \text {. matuvama }\end{array}$ & & & & & $\begin{array}{l}23.4 \\
24.9\end{array}$ & $\begin{array}{l}24.9 \\
26.4\end{array}$ & & & $\begin{array}{l}31.25 \\
40.75\end{array}$ & $\begin{array}{l}36.06 \\
45.54\end{array}$ & 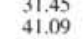 & $\begin{array}{l}41.09 \\
50.69\end{array}$ & $\begin{array}{l}32.93 \\
42.69\end{array}$ & $\begin{array}{l}42.69 \\
52.1\end{array}$ & & & & & & \\
\hline $\begin{array}{l}\text { B. R. matuyama } \\
\text { T. } R \text { praebergonii v, robustus }\end{array}$ & & & & & & & & & 58.7 & 59.2 & 58.64 & $\begin{array}{l}30.099 \\
69.71\end{array}$ & $\begin{array}{l}2.2 .19 \\
52.1\end{array}$ & 61.38 & 45.1 & 51.1 & 47.7 & 50.09 & & 53.74 \\
\hline B. P. doliolus & 14.75 & 19.3 & 9.1 & 13.95 & 36.49 & 36.4 & 27.95 & 41.19 & 64.26 & 65.25 & 69.71 & 78.36 & 64.9 & $\begin{array}{l}61.06 \\
65.6\end{array}$ & 60.6 & 62.1 & 59.19 & 69.09 & 53.74 & 63.64 \\
\hline T. T. convexa s.t. & & & 21.95 & 26.45 & 43.3 & 44.8 & & & 69.7 & 71.2 & 87.63 & 97.95 & 71.1 & 71.4 & 71.6 & 72.83 & 69.09 & 77.35 & 63.64 & 73.2 \\
\hline T. N. jouseae & 26.1 & 33.48 & & & 46.8 & 47.3 & 41.49 & 48.4 & 93.6 & 95.21 & 97.95 & 106.23 & 97.4 & 98 & 83.1 & 84.6 & 88.08 & 97.6 & 83.25 & 89.8 \\
\hline B. R. praebergonii s.l. & 26.1 & 33.48 & 18.62 & 28.66 & 47.3 & 49.8 & 48.4 & 59.97 & 110.7 & 110.9 & 97.95 & 116.52 & 106.26 & 115.91 & 92.68 & 101.56 & 90.4 & 93.4 & 92.28 & 102.19 \\
\hline $\begin{array}{l}\text { F. T. comexa v. convexa } \\
\text { B. A. elegans }\end{array}$ & & & & & 56.3 & 57.8 & & & 130.78 & & & & 131.9 & 132.8 & $\begin{array}{l}108.2 \\
113.1\end{array}$ & $\begin{array}{l}111.58 \\
121.05\end{array}$ & 107.1 & 116,44 & 102.19 & 111.51 \\
\hline T. N. cylindrica & & & & & 66.37 & 67.87 & & & 169.17 & 169.98 & 164.22 & 173.34 & 167.95 & 191.59 & 130.35 & 139.81 & 143.35 & 144.73 & & \\
\hline $\begin{array}{l}\text { B. N. jouseae } \\
\text { B. T. nestrupii }\end{array}$ & 33.48 & 34.2 & & & $\begin{array}{l}64.87 \\
73.3\end{array}$ & $\begin{array}{l}66.37 \\
74.55\end{array}$ & 69.85 & 79.57 & $\begin{array}{l}178.65 \\
202.98\end{array}$ & $\begin{array}{l}179.48 \\
203.8\end{array}$ & 173.34 & 183.34 & $\begin{array}{l}180 \\
204.3\end{array}$ & $\begin{array}{l}180.6 \\
204.7\end{array}$ & 157,9 & 160.9 & 164.13 & 167.9 & & \\
\hline T. T. miocenica & & & & & 78.3 & 79.8 & & & $\begin{array}{l}202.98 \\
20298\end{array}$ & 203.8 & & & 201.21 & 210.74 & 174.52 & 180.5 & 183.7 & 185.2 & & \\
\hline T. N. miocenia & 33.48 & 34.2 & 31.85 & 33.35 & 81.3 & 82.8 & & & 215.9 & 216.26 & & & 210.74 & 220.16 & & & & & & \\
\hline T. $N$. miocenica v, elongata & $\begin{array}{l}33.48 \\
33.48\end{array}$ & 34.2 & 28.85 & 31.85 & & & & & 216.26 & 225.9 & & & & & & & & & & \\
\hline $\begin{array}{l}\text { T. T. praecomvexa } \\
\text { B. T. miocenica }\end{array}$ & 33.48 & 34.2 & & & 84.07 & 93.61 & & & $\begin{array}{l}216.26 \\
231.71\end{array}$ & $\begin{array}{l}225.9 \\
232.12\end{array}$ & & & $\begin{array}{l}210.74 \\
230.06\end{array}$ & 220.16 & 202.97 & 203.4 & 196.2 & 198.7 & & \\
\hline $\begin{array}{l}\text { B. T. miocenica } \\
\text { B. T. comvera v. aspinosa }\end{array}$ & 34.2 & 35.7 & 31.85 & 33.35 & 84.07 & 93.61 & & & $\begin{array}{l}2331.71 \\
-171\end{array}$ & $\begin{array}{l}232.12 \\
232.12\end{array}$ & & & $\begin{array}{l}230.06 \\
230.06\end{array}$ & $\begin{array}{l}239.86 \\
239.86\end{array}$ & & & & & & \\
\hline $\begin{array}{l}\text { B. } T \text {. comvexa v. aspinosa } \\
\text { B. } T \text {, praeconvexa }\end{array}$ & 35.7 & 37.9 & & & & & & & 232.12 & 236.8 & & & 239.86 & 249.45 & & & & & & \\
\hline $\begin{array}{l}\text { T. N. porteri } \\
\text { B. N. mincenica }\end{array}$ & 37.9 & 40.2 & 38.18 & 47.56 & 83.89 & 97.3 & & & $\begin{array}{l}247.9 \\
253.2\end{array}$ & $\begin{array}{r}248.95 \\
253.56\end{array}$ & & & & & & & & & & \\
\hline T. R. paleacea & 37.9 & 40.2 & 38.18 & 47.56 & 0.09 & M. & & & 258.5 & 258.7 & & & & & & & & & & \\
\hline B. N. reinholdii & 40.2 & $\begin{array}{l}41.7 \\
43.7\end{array}$ & 38.18 & 47.56 & 110.4 & 112.36 & 108.02 & 122.49 & 274.9 & 275,12 & & & & & & & & & & \\
\hline $\begin{array}{l}\text { T. T. vabei Group } \\
\text { B. N. cylindrica }\end{array}$ & $\begin{array}{l}42.9 \\
43.7\end{array}$ & $\begin{array}{l}45.2 \\
45.1\end{array}$ & & & & & 100,02 & & 274.5 & 275.12 & & & & & & & & & & \\
\hline T. C. loeblichii & 49.7 & 52.52 & & & & & & & & & & & & & & & & & & \\
\hline B. T. burckliama & 49.7 & 52.52 & & & & & & & & & & & & & & & & & & \\
\hline B. C. loeblichii & 52.52 & $\begin{array}{l}62.06 \\
606\end{array}$ & & & 122.8 & 131.67 & 122.49 & 155.55 & 293.1 & 293.7 & & & & & & & & & & \\
\hline $\begin{array}{l}\text { T. D. simonsenii s.l. } \\
\text { T. A. moronensis }\end{array}$ & $\begin{array}{l}59 \\
62.7\end{array}$ & $\begin{array}{l}62.06 \\
64.2\end{array}$ & 57.2 & $\begin{array}{l}66,73 \\
66.73\end{array}$ & 122.0 & BStor & 122.49 & 150.5נ & & $293 . I$ & & & & & & & & & & \\
\hline B. A. ellipticus f. lanceolata & 811 & 90.27 & & & & & & & 302.38 & 312.68 & & & & & & & & & & \\
\hline $\begin{array}{l}\text { T. C. coscinodiscus } \\
\text { Tose }\end{array}$ & 81.1 & 90.27 & 76.3 & 85.78 & & & 144.56 & 155.55 & 320.8 & 321.1 & & & & & & & & & & \\
\hline B. R. paleacea v. elongata & 81.1 & 90.27 & & & & & & & 320.8 & 321.1 & & & & & & & & & & \\
\hline T. C. gigas v. diorama & & & & & 150.62 & 159.95 & & & 320.8 & 321.1 & & & & & & & & & & \\
\hline T. A. ingens & 109.59 & 118.95 & 114.15 & 123.62 & 169.69 & 170.3 & 174.53 & 177.3 & 336.5 & 338.78 & & & & & & & & & & \\
\hline $\begin{array}{l}\text { T. C. pulchellus } \\
\text { B. N. porteri }\end{array}$ & 109.59 & 118.95 & & & 159.95 & 169.69 & & & 338.78 & 341.53 & & & & & & & & & & \\
\hline T. C. lemperiv velicata & $\begin{array}{l}109.59 \\
10950\end{array}$ & 118.95 & 1441 & 12360 & & & & & 338.78 & 338.95 & & & & & & & & & & \\
\hline $\begin{array}{l}\text { T. A. californicus } \\
\text { B. C. gigas v. diorama }\end{array}$ & 109.59 & & 114.15 & 123.62 & & & & & 353.4 & 354.3 & & & & & & & & & & \\
\hline T. C. lewisiamus & 128.26 & $\begin{array}{l}137.82 \\
147.43\end{array}$ & 132.71 & 142.8 & 182.8 & 188.73 & 183.73 & 187.27 & 351.9 & 355.2 & & & & & & & & & & \\
\hline $\begin{array}{l}\text { 1. T. T. cippanae } \\
\text { B. T.moumoum }\end{array}$ & $\begin{array}{l}137 \\
137\end{array}$ & $\begin{array}{l}147.43 \\
147.43\end{array}$ & 142.8 & 152.16 & & & & & & & & & & & & & & & & \\
\hline B. D. simonsenii s.l. & & 157.07 & & & & & & & & & & & & & & & & & & \\
\hline T. C. peplum & 157.07 & 166.43 & & & 218.25 & 245.52 & & & & & & & & & & & & & & \\
\hline B. A. ellipticus & & $\begin{array}{r}175.83 \\
10558\end{array}$ & & & & & & & & & & & & & & & & & & \\
\hline $\begin{array}{l}\text { B. C. bissmos } \\
\text { T. A californicus }\end{array}$ & $\begin{array}{l}75.83 \\
194.69\end{array}$ & $\begin{array}{l}185.58 \\
204.27\end{array}$ & & & & & & & & & & & & & & & & & & \\
\hline B. A. ingens & 213.9 & 223.65 & & & & & & & & & & & & & & & & & & \\
\hline T. C. lewisamus v, simillis & 223.65 & 232.04 & & & & & & & & & & & & & & & & & & \\
\hline $\begin{array}{l}\text { T. T. fraga } \\
\text { B. C. peplum }\end{array}$ & $\begin{array}{l}261.66 \\
261.66\end{array}$ & $\begin{array}{l}270.7 \\
270.7\end{array}$ & & & 274.35 & 283.87 & & & & & & & & & & & & & & \\
\hline T. $R$, marylandicus & 261.66 & 270.7 & & & & & & & & & & & & & & & & & & \\
\hline
\end{tabular}

Note: $T=$ top or last occurrence of a species, $B=$ bottom or first occurrence of a species. 
Table 5. Depth constraints of stratigraphically useful events from eastern transect Holes 844B through 847D.

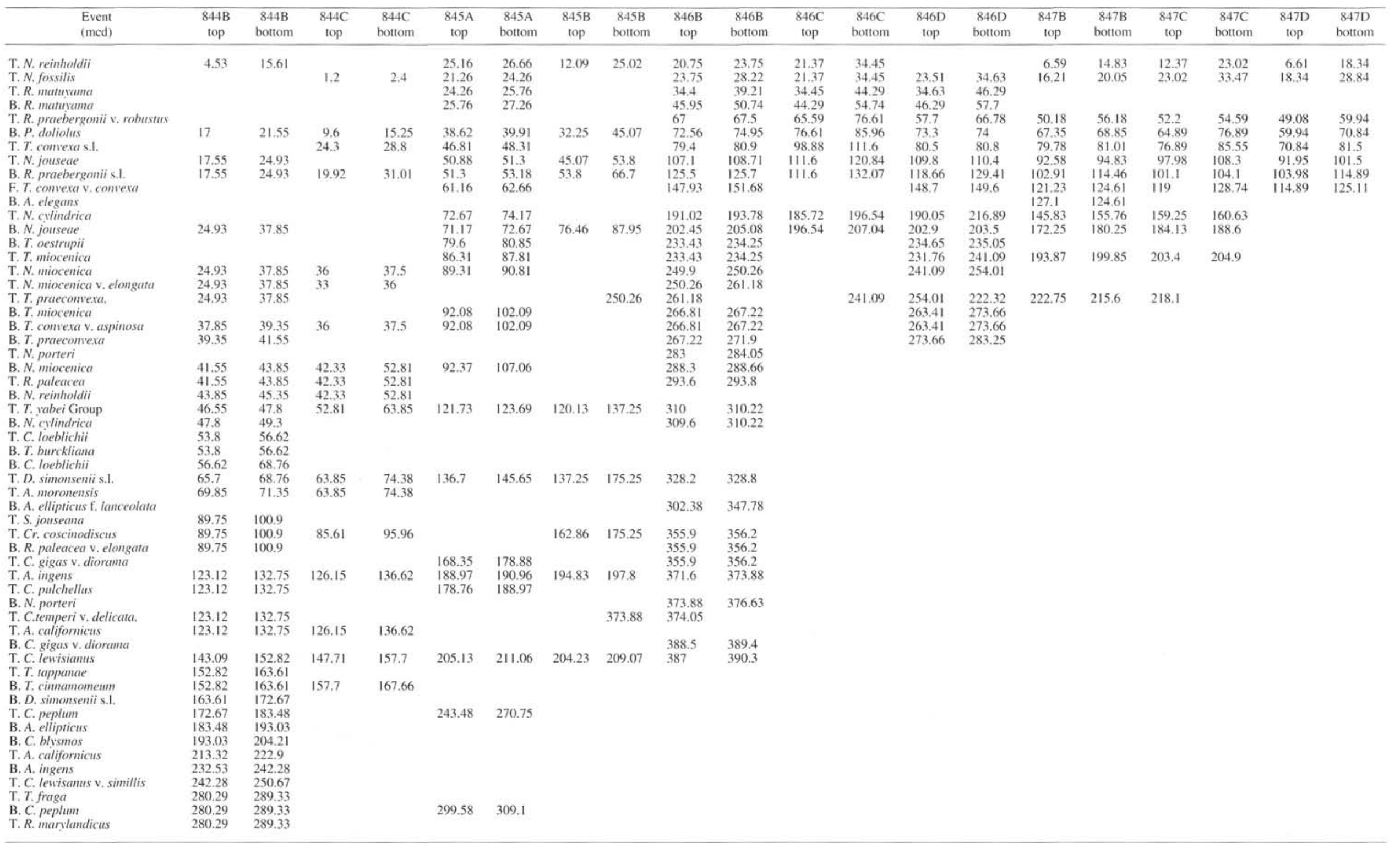


A

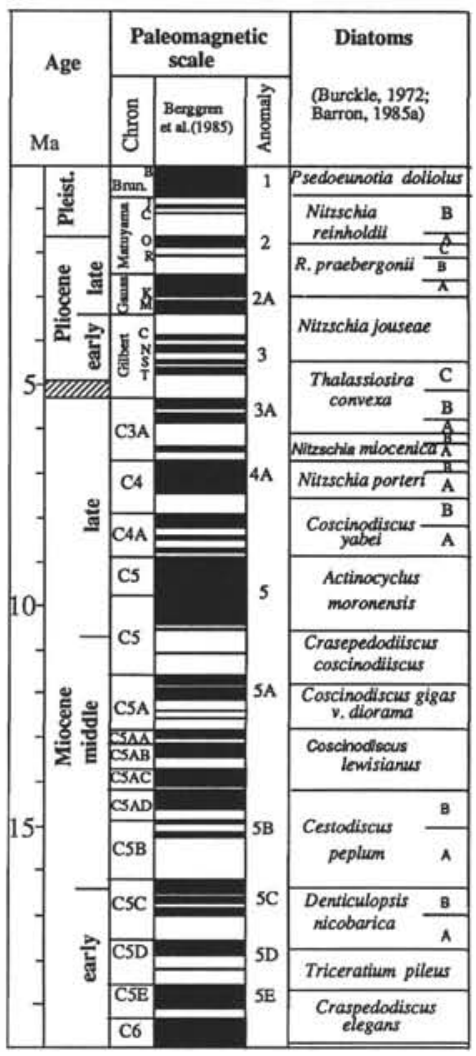

B

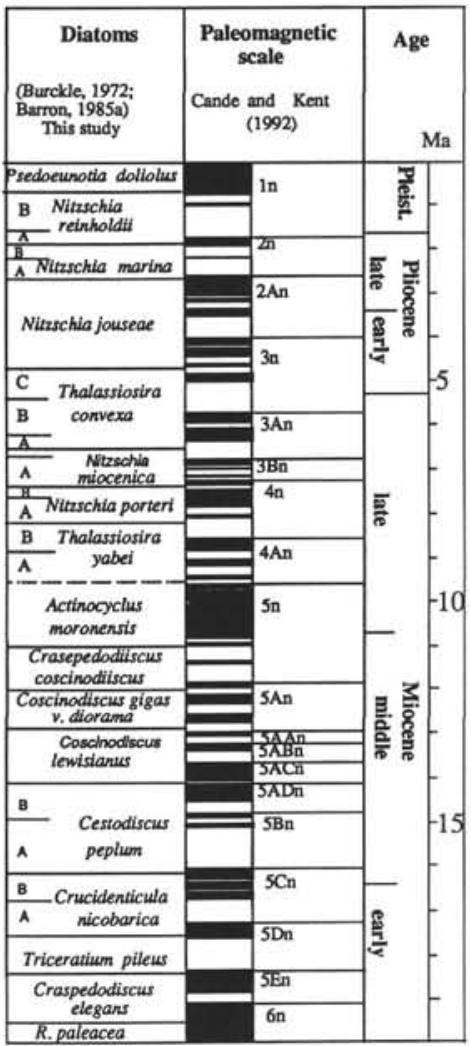

Figure 2. Comparison of the diatom zonation used here with the diatom zonation used aboard the ship (see Mayer, Pisias, Janecek, et al., 1992). A. Shipboard zonation has been calibrated to the chronology of Berggren et al. (1985). B. Zonation used here has been calibrated to the chronology of Cande and Kent (1992). See text for discussion of zones.

Thalassiosira burckliana in Sample 138-844B-6H-4, $120 \mathrm{~cm}$; and the LO of Denticulopsis simonsenii in Sample 138-844B-7H-CC. Samples $138-844 \mathrm{~B}-8 \mathrm{H}-2,60 \mathrm{~cm}$, through $-9 \mathrm{H}-\mathrm{CC}$ were assigned to the $A$. moronensis Zone, based on the occurrence of A. moronensis stratigraphically above the LO of Craspedodiscus coscinodiscus in Sample 138-844B-10H-CC. Sample 138-844B-13H-CC was assigned to the base of the $C$. coscinodiscus Zone on the basis of the occurrence of Thalassiosira brunii in this sample.

The diatom assemblage in the lowermost portion of Hole 844B is well preserved and abundant throughout the sequence, with most primary and secondary biostratigraphic indicators present. Samples from Core 138-844B-14H were assigned to the Coscinodiscus gigas var. diorama Zone. Samples 138-844B-15H-CC through -17H-CC represent the interval from the LO of Coscinodiscus lewisianus to the LO of Cestodiscus peplum and were assigned to the C. lewisianus Zone. Specimens of $C$. peplum occurred in samples examined from Cores 138-844B-19H through $-28 \mathrm{X}$, which allowed us to assign samples from these cores to the $C$. peplum Zone. The lowermost portion of Hole 844B has been placed in Subzone B of the Crucidenticula nicobarica Zone, based on the occurrence of Raphidodiscus marylandicus, Coscinodiscus blysmos, and Crucidenticula kanayae stratigraphically below the FO of $C$. peplum and stratigraphically above the LO of Thalassiosira bukryi.

\section{Site 845}

Diatoms are present throughout the Pleistocene (P. doliolus Zone) through the uppermost middle Miocene (Subzone B of the $C$. nicobarica Zone). Diatom preservation is generally good in the Pleistocene through upper Miocene portion of the sequence, but deteriorates as one proceeds lower in the sequence. Because of this, the stratigraphic indicator species were not always observed in samples examined from the lower sequence at Site 845 .

The shipboard biostratigraphy (Mayer, Pisias, Janecek, et al., 1992) has been substantially revised for Hole $845 \mathrm{~A}$, as discussed below. No revisions were made to the biostratigraphy for Hole $845 \mathrm{~B}$. Samples 138-845A-1H-CC through - $3 \mathrm{H}-5,120 \mathrm{~cm}$, and Sample 138-845B-1H$C C$ have been assigned to the $P$. doliolus Zone, based on the occurrence of $P$. doliolus stratigraphically above the LO of $N$. reinholdii.

Samples 138-845A-3H-6, $120 \mathrm{~cm}$, through $-4 \mathrm{H}-\mathrm{CC}$ contain both $N$. reinholdii and $P$. doliolus and were assigned to the $N$. reinholdii Zone. This zone could not be subdivided as $R$. praebergonii did not occur consistently in the upper portion of its range at this site. Samples $138-845 \mathrm{~A}-5 \mathrm{H}-1,30 \mathrm{~cm}$, through $-6 \mathrm{H}-2,120 \mathrm{~cm}$, represent the interval between the FO of $P$. doliolus and the LO of $N$. jouseae. This interval was assigned to the $N$. marina Zone, as used by Baldauf (1984). The LO of T. convexa was assigned to Sample 138-845A-5H$6,120 \mathrm{~cm}$, and marks the A/B subzonal boundary.

The stratigraphic interval from Samples $138-845 \mathrm{~A}-6 \mathrm{H}-2,120 \mathrm{~cm}$, to $-8 \mathrm{H}-1,27 \mathrm{~cm}$, represents the total range of $N$. jouseae. This interval was assigned to the $N$. jouseae Zone, as used by Baldauf (1984). Secondary biostratigraphic markers in this interval include the FO of Thalassiosira convexa var. convexa in Sample 138-845A-7H-1, 120 $\mathrm{cm}$, and the LO of $N$. cylindrica in Sample 138-845A-8H-3, $27 \mathrm{~cm}$.

Sample 138-845A-9H-CC contains the first observed specimens of Thalassiosira convexa var. aspinosa, which defines the base of the T. convexa Zone. The interval between Sample 138-845A-8H-2, 27 $\mathrm{cm}$, to $-9 \mathrm{H}-4,120 \mathrm{~cm}$, were assigned to Subzone C of this zone, based on the LO of Thalassiosira miocenica in Sample 138-845A-9H-4, $120 \mathrm{~cm}$. The Subzonal A/B boundary could not be placed, as $T$. 
Table 6. Sample constraints of stratigraphically useful events from western transect Holes 848B through 852D.

\begin{tabular}{|c|c|c|c|c|c|c|}
\hline Event & $848 \mathrm{~B}$ & $848 \mathrm{C}$ & 848D & $849 \mathrm{~B}$ & $849 \mathrm{C}$ & 849D \\
\hline T. N. reinholdii & $1-\mathrm{CC} / 2-\mathrm{CC}$ & $0 / 1-\mathrm{CC}$ & $1-\mathrm{CC} / 2-\mathrm{CC}$ & & & $1-\mathrm{CC} / 2-\mathrm{CC}$ \\
\hline T.N.fossilis & \multirow[t]{3}{*}{$1-\mathrm{CC} / 2-\mathrm{CC}$} & \multirow{3}{*}{$1-\mathrm{CC} / 2-\mathrm{CC}$} & \multirow[t]{3}{*}{$1-\mathrm{CC} / 2-\mathrm{CC}$} & $3-5,75 / 4-1,70$ & & $2-\mathrm{CC} / 3-\mathrm{CC}$ \\
\hline T. R. matuyama & & & & $3-5,75 / 4-1,70$ & & \\
\hline B. R. matuyama & & & & $4-1,70 / 4-3,80$ & & \\
\hline T. $R$. praebergoni v, robustus & $3-6,120 / 3-\mathrm{CC}$ & $2-\mathrm{CC} / 3-\mathrm{CC}$ & $2 \mathrm{H}-\mathrm{CC} / 3 \mathrm{H}-\mathrm{CC}$ & $5-4,70 / 5-5,70$ & 4-CC/5-1, 70 & 4-CC/5-CC \\
\hline B. P. doliolus & $4-1,120 / 4-2,121$ & $3-\mathrm{CC} / 4-1,40$ & $4 \mathrm{H}-2,100 / 4 \mathrm{H}-\mathrm{CC}$ & $6-3,15 / 6-5,70$ & $5-6,70 / 6-\mathrm{CC}$ & $5-\mathrm{CC} / 6-\mathrm{CC}$ \\
\hline T. T. convexa s.1. & $4-3,120 / 4-4,121$ & $4-1,40 / 4-2,40$ & $2 \mathrm{H}-\mathrm{CC} / 3 \mathrm{H}-\mathrm{CC}$ & $7-3,60 /-\mathrm{CC}$ & 6-CC $7-1,70$ & 6-CC $/ 7-\mathrm{CC}$ \\
\hline T.N.jouseae & $4-4,121 / 4-5,121$ & $4-2,40 / 4-4,40$ & & $8-2,60 / 8-4,60$ & $7-6,70 / 7-\mathrm{CC}$ & $6-\mathrm{CC} / 7-\mathrm{CC}$ \\
\hline B. R. praebergonii s.l. & $4-6,120 / 4-7,10$ & \multirow[t]{6}{*}{$4-4,40 / 4-\mathrm{CC}$} & \multirow[t]{3}{*}{$4 \mathrm{H}-7,40 / 4 \mathrm{H}-\mathrm{CC}$} & $9-2,60 / 9-4,60$ & $8-5,70 / 8-\mathrm{CC}$ & 7-CC/8-CC \\
\hline F. T. convexa v. convexa & \multirow[b]{2}{*}{$\begin{array}{l}5-1,120 / 5-2,121 \\
4-\mathrm{CC} / 5-4,121\end{array}$} & & & $11-1,70 / 11-2,70$ & $9-\mathrm{CC} / 10-\mathrm{CC}$ & 9-CC/10-CC \\
\hline B. A. elegans & & & & & & \\
\hline T. N. cylindrica & $5-4,121 / 5-\mathrm{CC}$ & & $4 \mathrm{H}-\mathrm{CC} / 5 \mathrm{H}-\mathrm{CC}$ & $15 \mathrm{X}-1,70 / 15 \mathrm{X}-3,70$ & & \\
\hline $\begin{array}{l}\text { B. } N . \text { jouseae } \\
\text { B. } T \text {. oestrupii }\end{array}$ & \multirow[t]{2}{*}{$6-2,120 / 6-4,121$} & & $4 \mathrm{H}-\mathrm{CC} / 5 \mathrm{H}-\mathrm{CC}$ & $16 \mathrm{X}-1,70 / 16 \mathrm{X}-3,70$ & & $16 \mathrm{X}-1,70 / 16 \mathrm{X}-2,70$ \\
\hline $\begin{array}{l}\text { B. T. oestrupii } \\
\text { T. T. miocenica }\end{array}$ & & & & $20 \mathrm{X}-2,70 / 20 \mathrm{X}-6,70$ & & \\
\hline T. N.miocenica & $7-2,120 / 7-\mathrm{CC}$ & \multirow{8}{*}{$6-\mathrm{CC} / 7-\mathrm{CC}$} & \multirow{8}{*}{$\begin{array}{l}6 \mathrm{H}-\mathrm{CC} / 7 \mathrm{H}-\mathrm{CC} \\
6 \mathrm{H}-\mathrm{CC} / 7 \mathrm{H}-\mathrm{CC}\end{array}$} & $21 \mathrm{X}-6,24 / 21 \mathrm{X}-\mathrm{CC}$ & & $20 \mathrm{X}-\mathrm{CC} / 21 \mathrm{X}-\mathrm{CC}$ \\
\hline T. N. miocenica v. elongata & $7-5,120 / 8-2,121$ & & & $21 \mathrm{X}-6,24 / 21 \mathrm{X}-\mathrm{CC}$ & & $20 \mathrm{X}-\mathrm{CC} / 21 \mathrm{X}-\mathrm{CC}$ \\
\hline T. T. praeconvexa & $7-5,120 / 8-2,121$ & & & $21 \mathrm{X}-6,24 / 21 \mathrm{X}-\mathrm{CC}$ & & $20 \mathrm{X}-\mathrm{CC} / 21 \mathrm{X}-\mathrm{CC}$ \\
\hline T. R. praepaleacea & $8-2,121 / 8-3,121$ & & & & & \\
\hline B. T. miocenica & $8-3,121 / 8-4,70$ & & & $24 \mathrm{X}-3,60 / 24 \mathrm{X}-6,60$ & & $23 \mathrm{X}-\mathrm{CC} / 24 \mathrm{X}-\mathrm{CC}$ \\
\hline B. $T$. convexa $\mathrm{v}$. aspinosa & $8-3,121 / 8-4,70$ & & & $24 \mathrm{X}-3,60 / 24 \mathrm{X}-6,60$ & & $23 \mathrm{X}-\mathrm{CC} / 24 \mathrm{X}-\mathrm{CC}$ \\
\hline B. B. praeconvexa & $8-4,120 / 8-5,121$ & & & $26 \mathrm{X}-\mathrm{CC} / 27 \mathrm{X}-2,70$ & & \\
\hline T. N.porteri & \multirow{18}{*}{$\begin{array}{l}9-2,36 / 9-2,96 \\
9-2,36 / 9-2,96\end{array}$} & & & & & \\
\hline B. N. miocenica & & \multirow{17}{*}{$7-\mathrm{CC} / 8-\mathrm{CC}$} & \multirow{17}{*}{$\begin{array}{l}7 \mathrm{H}-\mathrm{CC} / 8 \mathrm{H}-\mathrm{CC} \\
7 \mathrm{H}-\mathrm{CC} / 8 \mathrm{H}-\mathrm{CC}\end{array}$} & $28 \mathrm{X}-4,60 / 28 \mathrm{X}-5,70$ & & \multirow{10}{*}{$\begin{array}{l}28 \mathrm{X}-\mathrm{CC} / 29 \mathrm{X}-\mathrm{CC} \\
29 \mathrm{X}-\mathrm{CC} / 30 \mathrm{X}-3,60 \\
29 \mathrm{X}-\mathrm{CC} / 30 \mathrm{X}-3,60\end{array}$} \\
\hline T. R. paleacea & & & & & & \\
\hline T. T. burckliana & & & & $31 \mathrm{X}-1,70 / 31 \mathrm{X}-2,70$ & & \\
\hline B. N. reinholdii & & & & $29 \mathrm{X}-2,70 / 29 \mathrm{X}-5,70$ & & \\
\hline T. A. ellipticus var javanica & & & & $29 \mathrm{X}-1,70 / 29 \mathrm{X}-3,70$ & & \\
\hline T. T. yabei Group & & & & $31 \mathrm{X}-1,70 / 31 \mathrm{X}-2,70$ & & \\
\hline B. N. cylindrica & & & & $30 \times-4,55 / 30 \times-5,70$ & & \\
\hline T. C. loeblichii & & & & & & \\
\hline B. T. burckliana & & & & $31 X-1,70 / 31 X-2,70$ & & \\
\hline B. C. loeblichii & & & & & & \\
\hline T. D. simonsenii & & & & $32 \mathrm{X}-5,70 / 33 \mathrm{X}-1,70$ & & $31 \mathrm{X}-\mathrm{CC} / 32 \mathrm{X}-\mathrm{CC}$ \\
\hline T. A. moronensis & & & & & & \\
\hline T. A. ellipticus & & & & & & \\
\hline T. S. jouseana & & & & & & \\
\hline T. C. coscinodiscus & & & & & & \\
\hline B. R. paleacea v. elongata & & & & & & \\
\hline T. C. gigas v. diorama & & & & & & \\
\hline
\end{tabular}

Notes: $\mathrm{T}=$ top or last occurrence of a species, $\mathrm{B}=$ bottom or first occurrence of a species, $\mathrm{CC}=$ core catcher, and $\mathrm{X}=$ extended core barrel. All samples were taken from APC-cored material unless noted otherwise.

praeconvexa, the subzonal indicator, did not occur consistently at this site. The interval from Samples 138-845A-10H-CC through -11H-1, $29 \mathrm{~cm}$, was assigned to the Nitzschia porteri Zone. The inconsistent occurrence of $T$. praeconvexa similarly did not allow us to place the A/B subzonal boundary of this zone.

The LO of T. yabei occurs in Sample 138-845A-12H-CC and marks the base of the $N$. porteri Zone and the top of the T. yabei Zone. The base of the T. yabei Zone typically is defined by the LO of $A$. moronensis (Barron, 1985a). As previously discussed, this species has a scattered occurrence in the upper portion of its stratigraphic range and is not always a useful zonal indicator for the Leg 138 sediments. We used the LO of $D$. simonsenii as a secondary indicator for the base of the T. yabei Zone. This usage is supported by Barron (1985a), who on the basis of DSDP Leg 85 results, indicated that the LO of D. simonsenii approximates the LO of A. moronensis. Sample $138-845 \mathrm{~A}-14 \mathrm{H}-1,120 \mathrm{~cm}$, was assigned to the base of the $T$. yabei Zone using this secondary marker.

Preservation and abundance of diatoms deteriorates in the lower portion of Hole 845A. Because of this, placement of stratigraphic indicators was less reliable. The $\mathrm{LO}$ of $C$. coscinodiscus could not be determined in Hole 845A, but has been placed in Sample 138-845B$16 \mathrm{H}-\mathrm{CC}$. Likewise, the FO of Thalassiosira brunii could not be placed in either hole. The LO of C. lewisianus, which marks the top of the $C$. lewisianus Zone, occurs in the interval between Samples 138-845A-20H-3, $120 \mathrm{~cm}$, and -20H-CC and between Samples 138$845 \mathrm{~A}-19 \mathrm{H}-\mathrm{CC}$ and $-20 \mathrm{H}-3,120 \mathrm{~cm}$.

Cestodiscus peplum occurs in Samples 138-845A-26X-CC through -29X-CC, which allowed us to assign these samples to the $C$. peplum Zone. The A/B subzonal boundary could not be placed. The occurrence of $C$. nicobarica and Crucidenticula kanayae without $C$. peplum in Samples 138-845A-30X-CC and -31X-2, $1 \mathrm{~cm}$, suggests that these samples are equivalent to the $C$. nicobarica Zone. Diatoms were not observed below Sample 138-845A-31X-2, $11 \mathrm{~cm}$.

\section{Site 846}

The recovered Pleistocene ( $N$. reinholdii Zone) through middle Miocene ( $C$. lewisianus Zone) sequence generally contains common and well-preserved diatoms. Revisions to the shipboard diatom biostratigraphy (Mayer, Pisias, Janecek, et al., 1992) were extensive for the upper 30 cores from Hole $846 \mathrm{~B}$ and for the 26 cores from Hole $846 \mathrm{D}$. Only minimal changes were made to the shipboard stratigraphy for the lower portion of Hole 846B and Holes 846A and 846C.

One core was recovered from Hole 846A. The occurrence of $P$. doliolus without $N$. reinholdii allowed us to assign samples from this core to be assigned to the $P$. doliolus Zone. Samples 138-846B-1H$\mathrm{CC}$ through $-3 \mathrm{H}-3,70 \mathrm{~cm}$, and Samples 138-846D-1H-CC through $-2 \mathrm{H}-\mathrm{CC}$ also were assigned to this zone. Samples $138-846 \mathrm{~B}-3 \mathrm{H}-4,70$ $\mathrm{cm}$, through $-7 \mathrm{H}-\mathrm{CC}$ and Samples 138-846D-3H-CC through -7H-5, $10 \mathrm{~cm}$, were assigned to the $N$. reinholdii Zone. This zonal assignment is based on the co-occurrence of $P$. doliolus and $N$. reinholdii in these samples.

The interval from Samples 138-846B-7H-CC through $-11 \mathrm{H}-2$, $121 \mathrm{~cm}$, represents the interval between the $\mathrm{FO}$ of $P$. doliolus and the LO of $N$. jouseae and was assigned to the $N$. marina Zone. The LO of $T$. convexa in Sample 138-846B-8H-5, $120 \mathrm{~cm}$, marks the A/B subzonal boundary. The consistent occurrence of $R$. praebergonii at Site 846 also allowed for recognition of the $R$. praebergonii Zone, 
Table 6 (continued).

\begin{tabular}{|c|c|c|c|c|c|c|c|}
\hline $850 \mathrm{~A}$ & $850 \mathrm{~B}$ & $851 \mathrm{~B}$ & $851 \mathrm{C}$ & $851 \mathrm{E}$ & $852 \mathrm{~B}$ & $852 \mathrm{C}$ & $852 \mathrm{D}$ \\
\hline $\begin{array}{l}1-\mathrm{CC} / 2-\mathrm{CC} \\
1-\mathrm{CC} / 2-\mathrm{CC}\end{array}$ & $\begin{array}{l}1-2,80 / 1-\mathrm{CC} \\
1-\mathrm{CC} / 2-\mathrm{CC}\end{array}$ & $\begin{array}{l}1-\mathrm{CC} / 2-2,25 \\
2-4,25 / 2-6,25\end{array}$ & $1-\mathrm{CC} / 2-\mathrm{CC}$ & $\begin{array}{l}1-\mathrm{CC} / 2-1,80 \\
2-1,80 / 2-3,80\end{array}$ & $1-2,25 / 2-2,25$ & $1-\mathrm{CC} / 2-\mathrm{CC}$ & $1-\mathrm{CC} / 2-\mathrm{CC}$ \\
\hline $\begin{array}{l}4-3,60 / 4-4,60 \\
5-2,60 / 5-5,60 \\
5-6,65 / 5-7,35 \\
5-\mathrm{CC} / 6-\mathrm{CC} \\
6-\mathrm{CC} / 7-\mathrm{CC} \\
7-4,61 / 7-\mathrm{CC} \\
7-\mathrm{CC} / 8-\mathrm{CC}\end{array}$ & $\begin{array}{l}41,60 / 4-2,70 \\
4-4,70 / 4-6,100 \\
5-6,65 / 5-7,35 \\
6-1,80 / 6-3,80 \\
6-C C / 7-C C \\
7-4,61 / 7-C C \\
8-2,80 / 8-C C \\
10-C C / 11 \text { X-CC } \\
11 \text { X-CC/12X-CC }\end{array}$ & $\begin{array}{l}3-\mathrm{CC} / 4-1,25 \\
4-\mathrm{CC} / 5-1,75 \\
5-1,75 / 5-3,75 \\
5-\mathrm{CC} / 6-3,1 \\
6-3,1 / 6-5,1 \\
7-5,1 / 7-6,1 \\
7-\mathrm{CC} / 8-\mathrm{CC} \\
9-\mathrm{CC} / 10-\mathrm{CC} \\
9-\mathrm{CC} / 10-\mathrm{CC}\end{array}$ & $\begin{array}{l}\text { 2-CC/3-CC } \\
3-\mathrm{CC} / 4-\mathrm{CC} \\
4-\mathrm{CC} / 5-\mathrm{CC} \\
4-\mathrm{CC} / 5-\mathrm{CC} \\
5-\mathrm{CC} / 6-\mathrm{CC} \\
6-\mathrm{CC} / 7-\mathrm{CC} \\
\\
8-\mathrm{CC} / 9-\mathrm{CC} \\
8-\mathrm{CC} / 9-\mathrm{CC}\end{array}$ & $\begin{array}{l}2-\mathrm{CC} / 3-\mathrm{CC} \\
3-\mathrm{CC} / 4-\mathrm{CC} \\
4-\mathrm{CC} / 5-2,90 \\
5-4,90 / 5-6,90 \\
6-2,90 / 6-6,90 \\
7-4,100 / 7-5,78 \\
\\
9-2,90 / 9-4,63 \\
9-4,63 / 9-5,141\end{array}$ & $\begin{array}{l}2-\mathrm{CC} / 3-2,25 \\
3-5,25 / 3-6,25 \\
\\
3-\mathrm{CC} / 4-2,25 \\
4-4,25 / 4-6,25 \\
5-2,5 / 5-4,25 \\
4-2,25 / 4-4,25 \\
6-4,120 / 6-5,120 \\
6-4,120 / 6-5,120\end{array}$ & $\begin{array}{l}2-\mathrm{CC} / 3-\mathrm{CC} \\
2-\mathrm{CC} / 3-\mathrm{CC} \\
3-\mathrm{CC} / 4-\mathrm{CC} \\
3-\mathrm{CC} / 4-2,25 \\
4-\mathrm{CC} / 5-4,1 \\
5-4,1 / 5-\mathrm{CC} \\
5-\mathrm{CC} / 6-\mathrm{CC} \\
5-\mathrm{CC} / 6-\mathrm{CC}\end{array}$ & $\begin{array}{l}2-\mathrm{CC} / 3-\mathrm{CC} \\
2-\mathrm{CC} / 3-\mathrm{CC} \\
2-\mathrm{CC} / 3-\mathrm{CC} \\
3-\mathrm{CC} / 4-\mathrm{CC}\end{array}$ \\
\hline \multirow{9}{*}{ 7-CC/8-CC } & $\begin{array}{l}18 X-2,52 / 18 X-3,51 \\
19 X-2,80 / 19 X-3,80\end{array}$ & $\begin{array}{l}13-\mathrm{CC} / 14-2,70 \\
14-5,70 / 14-6,70 \\
15-2,90 / 15-6,20\end{array}$ & $\begin{array}{l}12-\mathrm{CC} / 13-\mathrm{CC} \\
13-\mathrm{CC} / 14-\mathrm{CC} \\
8-2,25 / 8-6,25\end{array}$ & $13-\mathrm{CC} / 14-\mathrm{CC}$ & $\begin{array}{l}7-4,25 / 8-2,25 \\
8-2,25 / 8-6,25 \\
8-2,25 / 8-6,25\end{array}$ & $7-\mathrm{CC} / 8-\mathrm{CC}$ & \\
\hline & $\begin{array}{l}21 \mathrm{X}-\mathrm{CC} / 22 \mathrm{X}-3,80 \\
21 \mathrm{X}-\mathrm{CC} / 22 \mathrm{X}-3,80 \\
22 \mathrm{X}-\mathrm{CC} / 23 \mathrm{X}-2,60\end{array}$ & $\begin{array}{l}15-\mathrm{CC} / 16 \mathrm{X}-4,80 \\
15-\mathrm{CC} / 16 \mathrm{X}-4,80 \\
16 \mathrm{X}-\mathrm{CC} / 17 \mathrm{X}-\mathrm{CC}\end{array}$ & & $\begin{array}{l}15 \mathrm{X}-4,50 / 15 \mathrm{X}-\mathrm{CC} \\
15 \mathrm{X}-4,50 / 15 \mathrm{X}-\mathrm{CC} \\
15 \mathrm{X}-\mathrm{CC} / 16 \mathrm{X}-\mathrm{CC}\end{array}$ & $\begin{array}{l}8-6,25 / 9-2,25 \\
9-2,25 / 9-3,75 \\
8-6,25 / 9-2,25 \\
8-6,25 / 9-2,25 \\
9-\mathrm{CC} / 10-2,25\end{array}$ & & \\
\hline & $\begin{array}{l}26 \mathrm{X}-1,80 / 26 \mathrm{X}-2,58 \\
26 \mathrm{X}-2,60 / 26 \mathrm{X}-3,58 \\
26 \mathrm{X}-\mathrm{CC} / 27 \mathrm{X}-\mathrm{CC}\end{array}$ & $\begin{array}{l}18 X-C C / 19 X-2,80 \\
19 X-2,80 / 19 X-3,80\end{array}$ & & $17 \mathrm{X}-\mathrm{CC} / 18 \mathrm{X}-\mathrm{CC}$ & $\begin{array}{l}9-2,25 / 9-3,75 \\
9-6,25,10-2,25 \\
10-6,25 / 11-4,25 \\
10-2,25 / 10-6,25\end{array}$ & & $\begin{array}{l}\text { 8-CC/9-CC } \\
8-\mathrm{CC} / 9-\mathrm{CC}\end{array}$ \\
\hline & $27 \mathrm{X}-6,62 / 27 \mathrm{X}-7,20$ & $20 \mathrm{X}-\mathrm{CC} / 21 \mathrm{X}-\mathrm{CC}$ & & & $9-4,25 / 10-2,25$ & 9-CC/10-CC & 9-CC/10-CC \\
\hline & $\begin{array}{l}28 \mathrm{X}-1,5 / 28 \mathrm{X}-3,23 \\
28 \mathrm{X}-3,23 / 28 \mathrm{X}-\mathrm{CC}\end{array}$ & $\begin{array}{l}21 \mathrm{X}-\mathrm{CC} / 22 \mathrm{X}-\mathrm{CC} \\
21 \mathrm{X}-\mathrm{CC} / 22 \mathrm{X}-\mathrm{CC}\end{array}$ & & $\begin{array}{l}21 \mathrm{X}-\mathrm{CC} / 22 \mathrm{X}-\mathrm{CC} \\
21 \mathrm{X}-\mathrm{CC} / 22 \mathrm{X}-\mathrm{CC}\end{array}$ & $\begin{array}{l}10-6,25 / 11-4,25 \\
10-2,25 / 10-6,25\end{array}$ & $9-\mathrm{CC} / 10-\mathrm{CC}$ & 9- $\mathrm{CC} / 10-\mathrm{CC}$ \\
\hline & $30 \mathrm{X}-\mathrm{CC} / 31 \mathrm{X}-\mathrm{CC}$ & & & & $11-4,25 / 11-6,25$ & & \\
\hline & $\begin{array}{l}32 X-4,80 / 32 X-5,86 \\
32 X-C C / 33 X-1,70\end{array}$ & $\begin{array}{l}24 \mathrm{X}-\mathrm{CC} / 25 \mathrm{X}-1,80 \\
25 \mathrm{X}-2,80 / 25 \mathrm{X}-7,25\end{array}$ & & $24 \mathrm{X}-\mathrm{CC} / 25 \mathrm{X}-\mathrm{CC}$ & & & \\
\hline & $40 X-2,55 / 40 X-4,48$ & & & & & & \\
\hline & & $31-\mathrm{CC} / 32-\mathrm{CC}$ & & & & & \\
\hline
\end{tabular}

as used by Barron (1985a, 1985b). Samples 138-846B-8H-1, 125 $\mathrm{cm}$, through $-12 \mathrm{H}-6,120 \mathrm{~cm}$, and Samples 138-846D-7H-5, $80 \mathrm{~cm}$, through $-11 \mathrm{H}-\mathrm{CC}$ were assigned to the $R$. praebergonii Zone. Samples $138-846 \mathrm{~B}-8 \mathrm{H}-1,125 \mathrm{~cm}$, and $-8 \mathrm{H}-4,20 \mathrm{~cm}$, were assigned to Subzone $\mathrm{C}$, based on the occurrence of $R$. praebergonii stratigraphically below the FO of $P$. doliolus (Sample 138-846B-7H-CC) and stratigraphically above the LO of $T$. convexa (Sample 138-846B-8H$5,120 \mathrm{~cm}$ ). Samples $138-846 \mathrm{~B}-8 \mathrm{H}-5,120 \mathrm{~cm}$, through $-11 \mathrm{H}-1,120$ $\mathrm{cm}$, were assigned to Subzone B of this zone on the basis of the co-occurrence of $T$. convexa and $R$. praebergonii without $N$. jouseae. Samples 138-846B-11H-2, $120 \mathrm{~cm}$, through $-12 \mathrm{H}-6,120 \mathrm{~cm}$, wear assigned to Subzone A of this zone, based on the co-occurrence of $N$. jouseae, $T$. convexa and $R$. praebergonii.

The interval from Samples 138-846B-11H-2, $121 \mathrm{~cm}$, through $-19 \mathrm{H}-\mathrm{CC}$ and Samples 138-846D-11H-1, $120 \mathrm{~cm}$, through $-19 \mathrm{X}-5$, $120 \mathrm{~cm}$, represent the total range of $N$. jouseae at this site and have been assigned to the $N$. jouseae Zone, as used by Baldauf (1984). Samples 138-846B-20H-1, $120 \mathrm{~cm}$, through $-25 \mathrm{X}-5,70 \mathrm{~cm}$, and Samples 138-846D-19H-6, $30 \mathrm{~cm}$, through -24X-CC were placed in the T. convexa Zone. Samples $138-845 \mathrm{~B}-20 \mathrm{H}-1,120 \mathrm{~cm}$, through $-22 \mathrm{X}-4,120 \mathrm{~cm}$, were assigned to Subzone C, which consists of the interval between the FO of $N$. jouseae and the LO of T. miocenica, and Samples 138-846B-22H-5, $120 \mathrm{~cm}$, through -23X-CC were assigned to Subzone B. The occurrence of $T$. praeconvexa, $N$. miocenica, T. miocenica, $T$. convexa, and $N$. miocenica var. elongata allowed us to assign Samples 138-846B-24X-CC through -25X-5, 70 $\mathrm{cm}$, to Subzone A of the T. convexa Zone. Samples 138-846B-25XCC through -27X-6, $50 \mathrm{~cm}$, and Samples 138-846D-25X-CC and
$-26 \mathrm{X}-\mathrm{CC}$ were assigned to the $N$. miocenica Zone, based on the occurrence of $N$. miocenica without $T$. convexa.

The interval from Samples 138-846B-27X-CC through -30X-1, $30 \mathrm{~cm}$, has been assigned to the $N$. porteri Zone. The LO of $T$. yabei group in Sample 138-846B-30X-1, $22 \mathrm{~cm}$, marks the top of the $T$. yabei Zone. Here, the $T$. yabei group consists of the plicate forms of Thalassiosira (i.e., T. yabei, T. plicatus). The base of the T. yabei Zone only tentatively has been placed, as the marker species, A. moronensis, is scattered in the upper part of its range at this site. Sample $138-846 \mathrm{~B}-32 \mathrm{X}-2,60 \mathrm{~cm}$, approximates the base of this zone. Placement of the zonal boundary at a depth equivalent to this sample is supported by the abundant occurrence of the secondary indicator $D$. simonsenii in this sample.

Assignment of samples to the $C$. coscinodiscus, C. gigas var. diorama, and $C$. lewisianus zones was similar to that of the shipboard biostratigraphy. Samples 138-846B-34X-CC through -36X-5, 103 $\mathrm{cm}$, were placed in the $C$. coscinodiscus Zone. Samples 138-846B$36-\mathrm{CC}$ through $-38 \mathrm{X}-1,90 \mathrm{~cm}$, were assigned to the $C$. gigas var. diorama Zone, and Samples 138-846B-38X-5, $41 \mathrm{~cm}$, and the lower portion of Core 138-846B-38X are equivalent to the $C$. lewisianus Zone. Diatoms were not observed in samples examined below Core 138-846B-38X.

\section{Site 847}

The Pleistocene ( $N$. reinholdii Zone) through uppermost Miocene (T. convexa Zone) sequence generally contains few-to-common, wellpreserved diatoms. Minimal changes to the shipboard biostratigraphy 
Table 7. Depth constraints of stratigraphically useful events from western transect Holes 848B through 852D.

\begin{tabular}{|c|c|c|c|c|c|c|c|c|c|c|c|c|c|c|}
\hline $\begin{array}{l}\text { Event } \\
\text { (mbsf) }\end{array}$ & $\begin{array}{c}848 \mathrm{~B} \\
\text { top }\end{array}$ & $\begin{array}{c}848 \mathrm{~B} \\
\text { bottom }\end{array}$ & $\begin{array}{c}848 \mathrm{C} \\
\text { top }\end{array}$ & $\begin{array}{c}848 \mathrm{C} \\
\text { bottom }\end{array}$ & $\begin{array}{l}848 D \\
\text { top }\end{array}$ & $\begin{array}{l}848 D \\
\text { bottom }\end{array}$ & $\begin{array}{c}849 \mathrm{~B} \\
\text { top }\end{array}$ & $\begin{array}{c}849 \mathrm{~B} \\
\text { bottom }\end{array}$ & $\begin{array}{l}849 \mathrm{C} \\
\text { top }\end{array}$ & $\begin{array}{c}849 \mathrm{C} \\
\text { bottom }\end{array}$ & $\begin{array}{l}849 \mathrm{D} \\
\text { top }\end{array}$ & $\begin{array}{c}849 \mathrm{D} \\
\text { bottom }\end{array}$ & $\begin{array}{l}850 \mathrm{~A} \\
\text { top }\end{array}$ & $\begin{array}{l}850 \mathrm{~A} \\
\text { bottom }\end{array}$ \\
\hline T. N. reinholdii & 2.23 & 12.27 & 0 & 5.47 & 8.64 & 20.23 & & & & & 8.36 & 22.97 & 7.68 & 16.74 \\
\hline T. N. fossilis & 2.23 & 12.3 & 5.47 & 17.7 & 8.64 & 20.23 & 22.95 & 26.4 & & & 22.97 & 32.79 & 7.68 & 16.74 \\
\hline T. R. matuyama & & & & & & & 22.95 & 26.4 & & & & & & \\
\hline B. R. matuyama & & & & & & & 26.4 & 29.5 & & & & & & \\
\hline T. R. pracbergonii & 20.5 & 21.73 & 17.7 & 28.06 & 20.23 & 30.51 & 40.4 & 41.9 & 37.83 & 39.7 & 41.48 & 51.9 & 30.3 & 31.8 \\
\hline B. $P$. doliolus & 22.53 & 24.03 & 28.06 & 28,46 & 32.5 & 39.76 & 47.81 & 51.7 & 47.3 & 49.03 & 51.9 & 60.92 & 38.3 & 42.8 \\
\hline T. T. convexa & 25.53 & 27.03 & 28.46 & 30.16 & & & 57.8 & 64.26 & 49.03 & 58.7 & 60.92 & 70.93 & 44.35 & 45.55 \\
\hline T. N. jousecie & 27.03 & 28.83 & 30.16 & 33.65 & & & 65.8 & 68.8 & 65.7 & 68.03 & 60.92 & 70.93 & 46.21 & 55.81 \\
\hline B. R. praebergonii & 30.03 & 30.42 & 33.65 & 38.82 & 39.4 & 39.76 & 75.3 & 78.3 & 74.3 & 77.44 & 70.93 & 79.2 & 55.81 & 65.22 \\
\hline F. T. comvexa v, convexa & 31.9 & 33.4 & נד, & & & ס & 92.49 & 94.1 & 86.58 & 96.62 & 89.76 & 99.05 & 65.11 & 70.11 \\
\hline B. A. elegans & 31.17 & 36.42 & & & & & & & & & & & 65.22 & 74.76 \\
\hline T. N. cylindrica & 36.42 & 40.53 & & & 39.76 & 50.75 & 131 & 134 & & & & & & \\
\hline B. $N$. jousecue & 43.1 & 46.1 & & & 39.76 & 50.75 & 140.7 & 143.7 & & & 145.3 & 146.7 & & \\
\hline \multicolumn{15}{|l|}{ B. $T$. oestrupii } \\
\hline T. T. miocenica & & & & & & & 179.9 & 185.9 & & & & & & \\
\hline T. N. miocenica & 52.4 & 59.77 & 59.88 & 70.81 & 61.4 & 71.87 & 195.04 & 196.61 & & & 191.7 & 201.14 & & \\
\hline T. N. miocenica v. elongata & 56.9 & 61.9 & & & 61.4 & 71.87 & 195.04 & 196.61 & & & 191.7 & 201.14 & & \\
\hline T. T. praecomexa & 56.9 & 61.9 & 70.81 & & & & 195.04 & 196.61 & & & 191.7 & 201.14 & & \\
\hline T. R. praepaleacea & 61.9 & 63.4 & & & & & & & & & & & & \\
\hline B. T. miocenica & 63.4 & 64.4 & & & & & 219.0 & 224.4 & & & 220.86 & 230.56 & & \\
\hline B. T. comrext & 63.4 & 64.4 & & & & & 219.9 & 224.4 & & & 220.86 & 230.56 & & \\
\hline \multirow{2}{*}{\multicolumn{15}{|c|}{ T. N.porteri }} \\
\hline & & & & & & & & & & & & & & \\
\hline B. N. miocenica & 70.56 & 71.15 & & & 71.87 & 82.92 & 260.1 & 262.3 & & & 259.27 & 268.44 & & \\
\hline T. R. paleacera & 70.58 & 71.15 & 70.81 & 81.41 & 71.87 & 82.92 & & & & & & & & \\
\hline T. T. hurckliana & & & & & & & 284.6 & 286.1 & & & & & & \\
\hline B. N. reinholdii & & & & & & & 270.8 & 275.3 & & & & & & \\
\hline T. A. ellipticus v. jaramicus & & & & & & & 269.3 & 272.3 & & & 268.44 & 278.44 & & \\
\hline T. T. yabei & & & & & & & 284.6 & 286.1 & & & 278.44 & 288.07 & & \\
\hline B. N. cylindrica & & & & & & & 308.25 & 309.0 & & & 278.44 & 288.07 & & \\
\hline \multicolumn{15}{|l|}{ T. C. loeblichii } \\
\hline B. T. burckliana & & & & & & & 284.6 & 286.1 & & & & & & \\
\hline \multicolumn{15}{|l|}{ B. C. loeblichii } \\
\hline T. D. hustediii & & & & & & & 295.8 & 308.25 & & & 297.7 & 307.49 & & \\
\hline \multicolumn{15}{|l|}{ T. A. moronensis } \\
\hline \multicolumn{15}{|l|}{ T. A. ellipticus } \\
\hline \multicolumn{15}{|l|}{ T. S. jouseana } \\
\hline \multicolumn{15}{|l|}{ T. C. coscinediscus } \\
\hline \multicolumn{15}{|l|}{ B. R. puleacea v. elongata } \\
\hline T. C. gigas v. diorama & & & & & & & & & & & & & & \\
\hline
\end{tabular}

Notes: $\mathrm{T}=$ top or last occurrence of a species, $\mathrm{B}=$ bottom or first occurrence of a species, and $\mathrm{CC}=$ core catcher sample. All samples are taken from APC-cored material unless noted otherwise.

(Mayer, Pisias, Janecek, et al., 1992) were completed. Samples examined from Core $1 \mathrm{H}$ of each hole contains $P$. doliolus without $N$. reinholdii. Because of this, these cores were placed in the $P$. doliolus Zone. Samples 138-847B-2H-6, $70 \mathrm{~cm}$, through -7H-5, $60 \mathrm{~cm}, 138-$ $847 \mathrm{C}-3 \mathrm{H}$ through $-6 \mathrm{H}-\mathrm{CC}$ and $-847 \mathrm{D}-3 \mathrm{H}-\mathrm{CC}$ through $-6 \mathrm{H}-\mathrm{CC}$ were assigned to the $N$. reinholdii Zone. This zonal assignment was based on the co-occurrence of $P$. doliolus and $N$. reinholdii. The consistent occurrence of $R$. praebergonii in samples from this site allowed us to recognize the $\mathrm{LO}$ of $R$. praebergonii and the $\mathrm{A} / \mathrm{B}$ subzonal boundary in Samples 138-847B-6H-4, $60 \mathrm{~cm}$; -847C-5H-CC; and -847D-6HCC, as used by Barron (1985a, 1985b).

Samples 138-847B-7H-5, $60 \mathrm{~cm}$, through $-10 \mathrm{H}-2,60 \mathrm{~cm}$, represent the interval between the FO of $P$. doliolus and the $\mathrm{LO}$ of $N$. jouseae and were assigned to the $N$. marina Zone of Baldauf (1984). The consistent occurrence of $R$. praebergonii at this site also allowed for usage of the R. praebergonii Zone, as used by Barron (1985a, 1985B), and permitted assignment of Samples 138-847B-7H-6, $60 \mathrm{~cm}$, through -10H-CC; 138-847C-7H-CC through $-10 \mathrm{H}-2,148 \mathrm{~cm}$; and 138-847D-7H-CC through $-10 \mathrm{H}-\mathrm{CC}$ to the $R$. praebergonii Zone. Samples 138-847B$7 \mathrm{H}-6,60 \mathrm{~cm}$, through $-8 \mathrm{H}-6,60 \mathrm{~cm}$, were assigned to Subzone C, based on the occurrence of $R$. praebergonii stratigraphically below the FO of $P$. doliolus (Sample 138-847B-7H-5, $60 \mathrm{~cm}$ ) and stratigraphically above the LO of $T$. convexa (Sample 138-847B-8H-CC). Samples 138-847B-8H-CC through -9H-CC were assigned to Subzone B of this zone, based on the co-occurrence of $T$. convexa and $R$. praebergonii without $N$. jouseae. Samples 138-847B-10H-2, $60 \mathrm{~cm}$, through $-10 \mathrm{H}-\mathrm{CC}$ were assigned to Subzone A, based on the co-occurrence of $N$. jouseae, $T$. convexa, and $R$. praebergonii.
Samples 138-847B-10H-2, $60 \mathrm{~cm}$, through $-18 \mathrm{H}-2,90 \mathrm{~cm} ; 138-$ $847 \mathrm{C}-10 \mathrm{H}-\mathrm{CC}$ through $-17 \mathrm{X}-\mathrm{CC}$; and 138-847D-10-4, $120 \mathrm{~cm}$, through $-12 \mathrm{H}-\mathrm{CC}$ were assigned to the $N$. jouseae Zone, as used by Baldauf (1984) on the basis of the consistent occurrence of $N$. jouseae stratigraphically throughout this sequence. Samples from the remaining portion of Holes $847 \mathrm{~B}$ and $847 \mathrm{C}$ were assigned to the Thalassiosira convexa Zone. The LO of T. praeconvexa in Samples 138847B-23X-1, $60 \mathrm{~cm}$, and $-847 \mathrm{C}-21 \mathrm{X}-5,50 \mathrm{~cm}$, marks the A/B subzonal boundary of this zone.

\section{Site 848}

The Pleistocene (N. reinholdii Zone) through upper Miocene (Nitzschia porteri Zone) sequence contains common and wellpreserved diatoms. The shipboard biostratigraphy (Mayer, Pisias, Janecek, et al., 1992) was completed at a spacing of $1.5 \mathrm{~m}$. Because of this, this stratigraphy was not refined onshore. Thus individual events are shown in Tables 6-8, but the reader is referred to Mayer, Pisias, Janecek, et al. (1992) for a detailed discussion of the biostratigraphy for this site. A brief summary of the biostratigraphy places samples from Cores $138-848 \mathrm{~B}-1 \mathrm{H}$ and $-1 \mathrm{H}$ in the $P$. doliolus Zone. The interval from Samples 138-848B-2H-CC through -4H-1, $120 \mathrm{~cm}$; $138-848 \mathrm{C}-2 \mathrm{H}-\mathrm{CC}$ through $-3 \mathrm{H}-\mathrm{CC}$; and $138-848 \mathrm{D}-2 \mathrm{H}-\mathrm{CC}$ through $-4 \mathrm{H}-2,100 \mathrm{~cm}$, were assigned to the $N$. reinholdii Zone. Samples 138-848B-4H-2, $121 \mathrm{~cm}$, through $-4 \mathrm{H}-4,121 \mathrm{~cm} ; 138-844 \mathrm{C} 4 \mathrm{H}-1,40$ $\mathrm{cm}$, through $-4 \mathrm{H}-2,40 \mathrm{~cm}$, represent the interval from the $\mathrm{FO}$ of $P$. doliolus to the LO of $N$. jouseae and were assigned to the $N$. marina Zone. The interval representing the total range of $N$. jouseae includes 
Table 7 (continued).

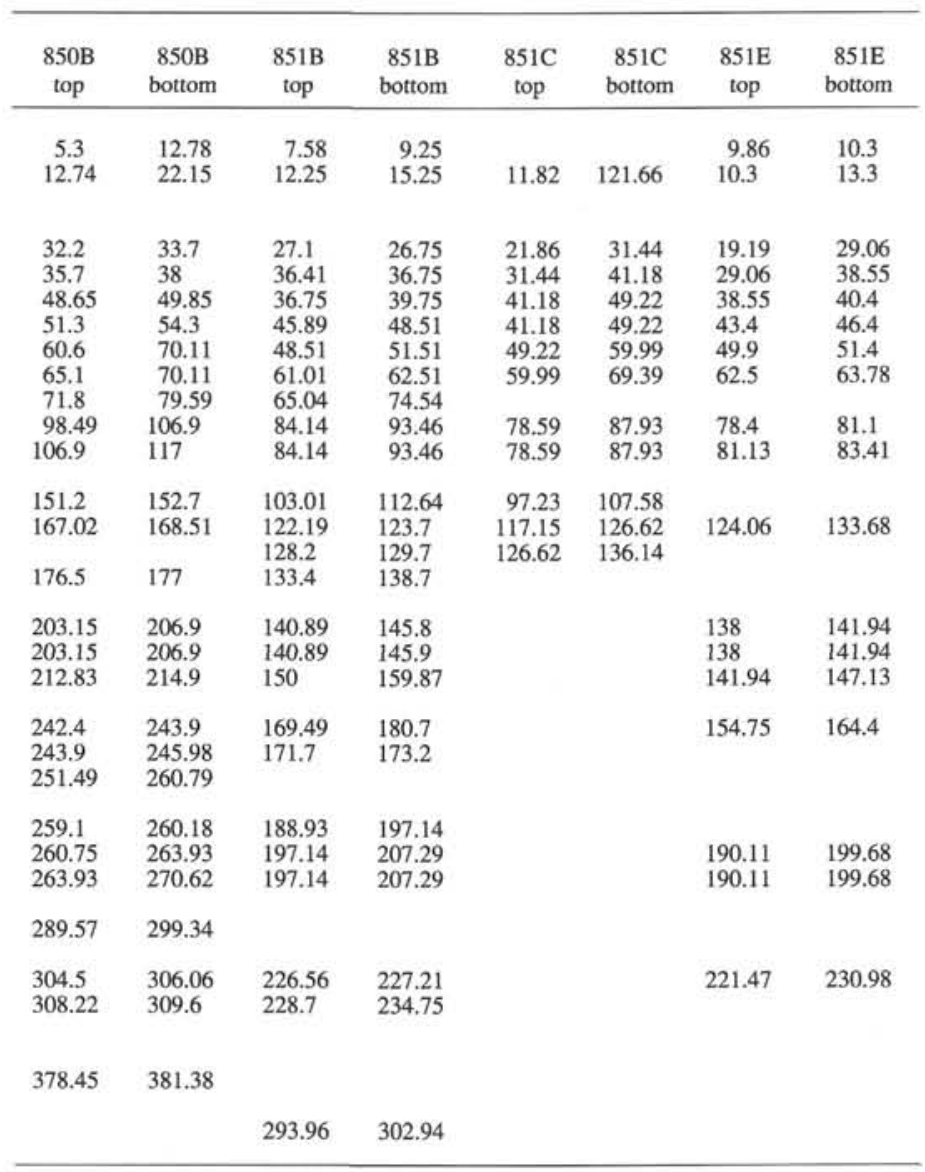

Samples $138-848 \mathrm{~B}-4 \mathrm{H}-5,121 \mathrm{~cm}$, through $-6 \mathrm{H}-2,120 \mathrm{~cm}$. This interval was assigned to the $N$. jouseae Zone of Baldauf (1984).

Samples 138-848B-6H-4, $120 \mathrm{~cm}$, through -8H-3, $120 \mathrm{~cm}$; 138$848 \mathrm{C}-6 \mathrm{H}-\mathrm{CC}$ and $-7 \mathrm{H}-\mathrm{CC}$; and $138-848 \mathrm{D}-4 \mathrm{H}-\mathrm{CC}$ were placed in the T. convexa Zone. Samples $138-848 \mathrm{~B}-8 \mathrm{H}-4,70 \mathrm{~cm}$, through $-9 \mathrm{H}-2,36$ $\mathrm{cm}$, and $138-848 \mathrm{D}-8 \mathrm{H}-\mathrm{CC}$ were assigned to the $N$. miocenica Zone, based on the occurrence of $N$. miocenica without $T$. convexa var. aspinosa. Preservation of diatoms in the interval equivalent to and stratigraphically below the $N$. porteri Zone (Cores $138-848 \mathrm{~B}-10 \mathrm{H}$ through $-11 \mathrm{H}, 138-848 \mathrm{C}-8 \mathrm{H}$ through $-9 \mathrm{H}$, and $138-848 \mathrm{D}-10 \mathrm{H})$ was generally poor to moderate. No zonal assignments for this interval was possible.

\section{Site 849}

APleistocene ( $N$. reinholdii Zone) to upper Miocene (Actinocyclus moronensis Zone) sequence was recovered having well-preserved diatoms that were consistently present. The shipboard biostratigraphy (Mayer, Pisias, Janecek, et al., 1992) for Hole 849B was substantially revised and is presented below. The biostratigraphy for Holes $849 \mathrm{C}$ and 849D has not been adjusted.

The biostratigraphic constraints for diatom events from Hole 849B are shown in Tables 6 through 8 . Although the LO of $N$. reinholdii could not be determined, the LO of Nitzschia fossilis in Sample 138$849 \mathrm{~B}-4 \mathrm{H}-1,70 \mathrm{~cm}$, indicates that the LO of $N$. reinholdii should stratigraphically occur above this sample.

The co-occurrence of $P$. doliolus and $N$. reinholdii in samples from Cores $138-849 \mathrm{D}-4 \mathrm{H}$ through $-6 \mathrm{H}-3,15 \mathrm{~cm}$, allowed us to assign these samples to the $N$. reinholdii Zone. The LO of $R$. praebergonii, which marks the A/B subzone, was placed in Sample 138-849D-5H-5, 70 $\mathrm{cm}$. The interval from the FO of $P$. doliolus in Sample 138-849D-6H$3,15 \mathrm{~cm}$, to the LO of $N$. jouseae in Sample 138-849D-8H-4, $60 \mathrm{~cm}$, was assigned to the $N$. marina Zone. The occurrence of $R$. praebergonii is scattered at this site, placing into question its stratigraphic reliability. The LO of T. convexa was placed in Sample 138-849D$7 \mathrm{H}-\mathrm{CC}$ and marks the A/B subzonal boundary.

The $N$. jouseae Zone extends from Sample 138-849D-8H-4, 60 $\mathrm{cm}$, through Sample 138-849D-16H-1, $70 \mathrm{~cm}$. Within this interval, the FO of $T$. convexa var. convexa was placed in Sample 138-849D$11 \mathrm{H}-1,70 \mathrm{~cm}$, and the LO of N. cylindrica was placed in Sample 138-849D-15H-3, $70 \mathrm{~cm}$.

The FO of T. convexa var. aspinosa in Sample 138-849D-24X-3, $60 \mathrm{~cm}$, allowed us to assign the interval from Sample 139-849D-16H$1,70 \mathrm{~cm}$, through $-24 \mathrm{X}-3,60 \mathrm{~cm}$, to the $T$. convexa Zone. The subzonal indicators permitted subdivision of this zone. The $\mathrm{LO}$ of $T$. miocenica, which marks the $\mathrm{B} / \mathrm{C}$ boundary, and the $\mathrm{LO}$ of $T$. praeconvexa, which marks the $\mathrm{A} / \mathrm{B}$ boundary, occur in the lowermost portion of Core 138-849D-21X. The LO of T. miocenica was placed in Sample 138$849 \mathrm{D}-21 \mathrm{X}-6,24 \mathrm{~cm}$, and the LO of T. praeconvexa was placed in Sample 138-849D-21X-CC.

The $N$. miocenica Zone extends from the base of the $T$. convexa Zone (Sample 138-849D-24X-3, $60 \mathrm{~cm}$ ) to the FO of N. miocenica in Sample 138-849D-28X-4, $60 \mathrm{~cm}$. The FO of $T$. praeconvexa is unreliable at this site; thus, we were unable to place the A/B subzonal boundary. The LO of T. yabei was placed in Sample 138-849D-31X$2,70 \mathrm{~cm}$, and marks the base of the $N$. porteri Zone. Within this zone, 
Table 8. Depth constraints of stratigraphically useful events from western transect Holes 848B through 852D.

\begin{tabular}{|c|c|c|c|c|c|c|c|c|c|c|c|c|c|c|}
\hline $\begin{array}{l}\text { Event } \\
\text { (mcd) }\end{array}$ & $\begin{array}{l}848 \mathrm{~B} \\
\text { top }\end{array}$ & $\begin{array}{l}848 \mathrm{~B} \\
\text { bottom }\end{array}$ & $\begin{array}{c}848 \mathrm{C} \\
\text { top }\end{array}$ & $\begin{array}{l}848 \mathrm{C} \\
\text { bottom }\end{array}$ & $\begin{array}{c}848 \mathrm{D} \\
\text { top }\end{array}$ & $\begin{array}{c}848 \mathrm{D} \\
\text { bottom }\end{array}$ & $\begin{array}{c}849 \mathrm{~B} \\
\text { top }\end{array}$ & $\begin{array}{l}849 \mathrm{~B} \\
\text { bottom }\end{array}$ & $\begin{array}{l}849 \mathrm{C} \\
\text { top }\end{array}$ & $\begin{array}{c}849 \mathrm{C} \\
\text { bottom }\end{array}$ & $\begin{array}{l}\text { 849D } \\
\text { top }\end{array}$ & $\begin{array}{l}849 \mathrm{D} \\
\text { bottom }\end{array}$ & $\begin{array}{c}850 \mathrm{~A} \\
\text { top }\end{array}$ & $\begin{array}{c}850 \mathrm{~A} \\
\text { bottom }\end{array}$ \\
\hline T.N. reinholdii & 2.43 & 15.07 & 0 & 5.47 & 8.44 & 18.28 & & & & & 8.36 & 22.97 & 7.68 & 18.28 \\
\hline T. N. fossilis & 2.42 & 15.07 & 5.47 & 15.45 & 8.44 & 18.28 & 15.63 & 19.9 & & & 22.97 & 32.79 & 7.68 & 18.28 \\
\hline \multicolumn{15}{|l|}{ T. R. matuyama } \\
\hline T. R. praebergoni & 24.45 & 25.68 & 15.45 & 25.06 & 18.28 & 27.81 & 40.4 & 43.4 & 37.83 & 49.03 & 41.48 & 51.9 & 33.3 & 34.8 \\
\hline B. P. doliolus & 26.88 & 28.38 & 25.06 & 24.9 & 29.9 & 37.16 & 47.81 & 51.7 & 49.03 & 57.4 & 51.9 & 60.92 & 42.1 & 46.6 \\
\hline T. T. convexa & 29.88 & 31.38 & 24.9 & 25.9 & 18.28 & 27.81 & 57.8 & 64.26 & 57.4 & 68.03 & 60.92 & 70.93 & 48.15 & 49.35 \\
\hline T. N. jouseae & 31.38 & 32.88 & 25.9 & 27.9 & & & 65.8 & 68.8 & 57.4 & 68.03 & 60.92 & 70.93 & 50.01 & 61.56 \\
\hline B. $R$. praebergonii & 34.38 & 34.77 & 27.9 & 34.57 & 37 & 37.16 & 75.3 & 78.3 & 60.03 & 77.44 & 70.93 & 79.2 & 61.56 & 72.57 \\
\hline F. $T$. convexa v. convexa & 37.55 & 39.05 & & & & & 92.49 & 94.1 & 86.58 & 96.62 & 89.76 & 99.05 & 72.46 & 72.57 \\
\hline B. A. elegans & 35.52 & 42.05 & & & & & & & & & & & 72.57 & 83.76 \\
\hline T. N. cylindrica & 42.05 & 46.18 & & & 37.16 & 46.75 & & & & & & & & \\
\hline B. N. jouseae & 58.57 & 61.57 & & & 37.16 & 46.75 & 140.7 & 149.53 & & & 144.41 & 154.2 & & \\
\hline \multicolumn{15}{|l|}{ B. $T$, oestrupii } \\
\hline \multicolumn{15}{|l|}{ T. T. miocenica } \\
\hline T. N. miocenica & 59.1 & 66.47 & 53 & 63.06 & 56.4 & 65.07 & 187.16 & 196.61 & & & 191.7 & 201.14 & & \\
\hline T. N. miocenica v. elongata & 63.6 & 69.35 & & & 56.4 & 65.07 & 187.16 & 196.61 & & & 191.7 & 201.14 & & \\
\hline T.T. praeconvexa & 63.6 & 69.35 & & & & & 187.16 & 196.61 & & & 191.7 & 201.14 & & \\
\hline T. $R$. praepaleacea & 69.35 & 70.85 & & & & & & & & & 220.86 & 230.56 & & \\
\hline B. T. miocenica & 70.85 & 71.85 & & & & & 219.9 & 224.4 & & & 220.86 & 230.56 & & \\
\hline B. T. convexa & 70.85 & 71.85 & & & & & 219.9 & 224.4 & & & & & & \\
\hline B. B. praeconvexa & 72.35 & 73.85 & & & & & 242 & 247.5 & & & 259.27 & 268.44 & & \\
\hline T. N. porteri & & & & & & & & & & & 268.44 & 278.44 & & \\
\hline B. N. miocenica & 80.16 & 80.75 & & & 65.07 & 75.42 & 269.78 & 275.08 & & & 278.44 & 288.07 & & \\
\hline T. R.paleacea & 80.16 & 80.75 & 63.06 & 72.31 & 65.07 & 75.42 & & & & & 278.44 & 288.07 & & \\
\hline B. $N$. reinholdii & & & & & & & & & & & 297.7 & 307.49 & & \\
\hline \multirow{2}{*}{\multicolumn{15}{|c|}{ B. N. marina }} \\
\hline & & & & & & & & & & & & & & \\
\hline B. N. cylindrica & & & & & & & 308.25 & 309.9 & & & & & & \\
\hline T. T, yabei & & & & & & & 308.25 & 309.9 & & & & & & \\
\hline \multicolumn{15}{|l|}{ T. C. loeblichii } \\
\hline \multicolumn{15}{|l|}{ B. T. burckliana } \\
\hline \multicolumn{15}{|l|}{ B. T. burcklina } \\
\hline \multicolumn{15}{|l|}{ B. C. loeblichii } \\
\hline T. D. hustedtii & & & & & & & 295.8 & 308.25 & & & & & & \\
\hline \multicolumn{15}{|l|}{ T. A. moronensis } \\
\hline \multicolumn{15}{|l|}{ T. A. ellipticus } \\
\hline \multicolumn{15}{|l|}{ T. S. jouseana } \\
\hline \multicolumn{15}{|l|}{ T. C. coscinodiscus } \\
\hline B. R. paleacea v. elongata & & & & & & & & & & & & & & \\
\hline T. C. gigas v. diorama & & & & & & & & & & & & & & \\
\hline
\end{tabular}

Note: $\mathrm{T}=$ top or last occurrence of a species, $\mathrm{B}$ = bottom or first occurrence of a species. Determined by Mayer, Pisias, Janecek, et al. (1992).

N. reinholdii has an FO in Sample 138-849D-29X-5, $70 \mathrm{~cm}$, and $T$. burckliana has an LO in Sample 138-849D-31X-1, $70 \mathrm{~cm}$.

The remaining portion of the hole was assigned to the $T . y a b e i$ Zone, based on the occurrence of T. yabei above the occurrence of A. moronensis. However, note that the scattered occurrence of $A$. moronensis at this site reduced its usefulness as a stratigraphic marker. Similar to other sites, we used the LO of $D$. simonsenii as an indicator for the base of this zone. Using this criterion, the lower portion of the hole approximates the base of the $T$. yabei as the LO of D. simonsenii was placed in Sample 138-849D-33X-1, $70 \mathrm{~cm}$.

\section{Site 850}

Pleistocene ( $N$. reinholdii Zone) to upper middle Miocene (Craspedodiscus coscinodiscus Zone) sediment was recognized. Few changes were made to the shipboard biostratigraphy for Hole $850 \mathrm{~B}$, discussed in Mayer, Pisias, Janecek, et al. (1992). No change was made to the stratigraphy of Hole $850 \mathrm{~A}$. The zonal boundaries were placed as follows.

The LO of $N$. reinholdii in Sample 138-850B-1H-CC allowed us to place Core $1 \mathrm{H}$ in the $P$. doliolus Zone. The FO of $P$. doliolus, which marks the base of the $N$. reinholdii Zone, was placed in Sample $138-850 \mathrm{~B}-4 \mathrm{H}-4,70 \mathrm{~cm}$. Within this zone, the LO of $R$. praebergonii has been tentatively placed in Sample 138-850B-4H-2, $70 \mathrm{~cm}$, allowing us to place the $\mathrm{A} / \mathrm{B}$ subzonal boundary in this sample. The $N$. marina Zone extends from the FO of $P$. doliolus to the LO of $N$. jouseae. The LO of $N$. jouseae was placed in Sample 138-850B-6H-3, $80 \mathrm{~cm}$. The $\mathrm{A} / \mathrm{B}$ subzonal boundary is defined at the $\mathrm{LO}$ of $T$. convexa, which was placed in Sample 138-850B-5H-7, $35 \mathrm{~cm}$.

The FO of $N$. jouseae, which marks the base of the $N$. jouseae Zone was placed in Sample 138-850B-11X-CC. Within this interval, the FO of $T$. convexa var. convexa was placed in Sample 138-850B$7 \mathrm{H}-4,61 \mathrm{~cm}$, and the LO of $N$. cylindrica was placed in Sample $138-850 \mathrm{~B}-11 \mathrm{X}-\mathrm{CC}$. The FO of $T$. convexa in Sample 138-850B-21X$\mathrm{CC}$ allowed us to place Samples 138-850B-12X-CC through -21X$\mathrm{CC}$ into the $T$. convexa Zone. The FO of $T$. miocenica, a secondary indicator for the base of this zone, also occurs in Sample 138-850B$21 \mathrm{X}-\mathrm{CC}$. The $\mathrm{C} / \mathrm{B}$ subzonal boundary, based on the LO of T. miocenica was not defined. The B/A subzonal boundary based on the $\mathrm{LO}$ of $T$. praeconvexa was placed in Sample 138-850B-19X-3, $80 \mathrm{~cm}$.

The $N$. miocenica Zone is defined as the interval between the FO of $T$. convexa and the FO of $N$. miocenica. This later species has an FO in Sample 138-850B-26X-1, $80 \mathrm{~cm}$, allowing for placement of the interval from Samples 138-850B-22X-3, $80 \mathrm{~cm}$, through -26X-1, 80 $\mathrm{cm}$, in this zone. The FO of $T$. praeconvexa in Sample 138-850B-22X$\mathrm{CC}$ allowed us to place the $\mathrm{A} / \mathrm{B}$ subzonal boundary in this sample.

The LO of Thalassiosira yabei in Sample 138-850B-28X-3, 23 $\mathrm{cm}$, allowed for placement of the interval from Samples 138-850B$26 \mathrm{X}-2,60 \mathrm{~cm}$, through $-28 \mathrm{X}-3,23 \mathrm{~cm}$, in the $N$. porteri Zone. The A/B subzonal boundary, defined as the LO of T. burckliana, was 
Table 8 (continued).

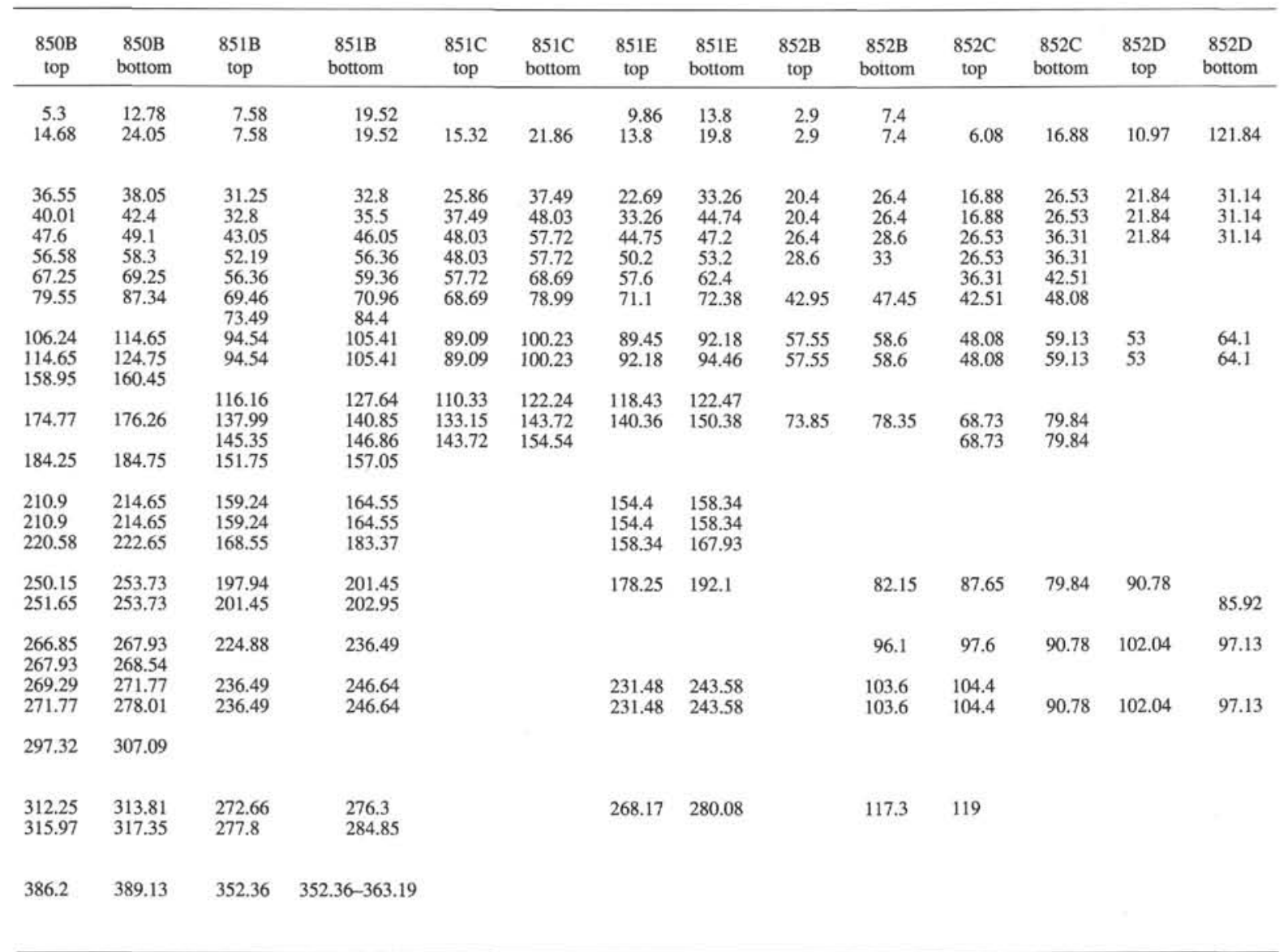

placed in Sample 138-850B-27X-CC. The FO of $N$. cylindrica occurs in Sample 138-850B-28X-3, $23 \mathrm{~cm}$, and consistently occurs at an interval equivalent to the LO of $T$. yabei.

Sample 138-850B-33X-1, $70 \mathrm{~cm}$, contains specimens of A. moronensis. Because of the scattered occurrence of this species in the top of its range, this occurrence permitted only tentative placement of the base of the T. yabei Zone. As at previous Leg 138 holes, the LO of $D$. simonsenii was used as a secondary indicator for this boundary. In Hole 850B, the LO of D. simonsenii was placed in Sample 138-850B$32 \mathrm{X}-5,86 \mathrm{~cm}$, slightly above the LO of A. moronenisis. The Subzone A/B boundary was placed in Sample 138-850B-30X-CC, based on the placement of the FO of $T$. burckliana in this sample. The base of the A. moronensis Zone was placed in Sample 138-850B-40X-4, 48 $\mathrm{cm}$, at the $\mathrm{LO}$ of $C$. coscinodiscus.

\section{Site 851}

Pleistocene ( $N$. reinholdii Zone) to uppermost middle Miocene ( $C$. coscinodiscus Zone) sediment was recovered at this site. Few changes to placement of the Hole 851B zonal boundaries, previously completed aboard the ship (Mayer, Pisias, Janecek, et al., 1992) were made.

The FO of $N$. reinholdii in Sample 138-851B-2H-2, $25 \mathrm{~cm}$, defines the base of the $P$. doliolus Zone and the FO of $P$. doliolus in Sample 138-851B-4H-CC, which marks the base of the $N$. reinholdii Zone. The LO of $R$. praebergonii has been tentatively placed in Sample $138-851 \mathrm{~B}-4 \mathrm{H}-1,25 \mathrm{~cm}$, allowing us to place the A/B sub- zonal boundary in this sample. The $N$. marina Zone extends from the FO of $P$. doliolus in Sample 138-851B-4H-CC to the LO of $N$. jouseae in Sample 138-851B-6H-3, $1 \mathrm{~cm}$. The A/B subzonal boundary defined at the LO of T. convexa was placed in Sample 138-851B-5H-3, $75 \mathrm{~cm}$.

The FO of $N$. jouseae defines the base of the $N$. jouseae Zone in Sample 138-851B-9H-CC. Within this interval, the FO of $T$. convexa var. convexa was placed in Sample 138-851B-7H-5, $1 \mathrm{~cm}$, and the LO of $N$. cylindrica was placed in Sample 138-851B-10H-CC. Thalassiosira convexa var. aspinosa has an FO in Sample 138-851B-15H-CC, allowing for the base of the $T$. convexa Zone equivalent to this sample. The $N$. miocenica zone is defined as the interval between the FO of $T$. convexa and the FO of $N$. miocenica. The FO of $N$. miocenica was placed in Sample 138-851B-18X-CC, allowing placement of the interval from Samples 138-851B-16X-4, $80 \mathrm{~cm}$, to $-18 \mathrm{X}-\mathrm{CC}$ in this zone. The FO of $T$. praeconvexa in Sample 138-851B-16H-CC permitting us to place the $\mathrm{A} / \mathrm{B}$ subzonal boundary in this sample.

The interval from Samples 138-851B-19X-2, $80 \mathrm{~cm}$, through $-22 \mathrm{X}-\mathrm{CC}$ was assigned to the $N$. porteri Zone. The subzonal $\mathrm{A} / \mathrm{B}$ boundary was not determined. Sample 138-851B-25X-7, $25 \mathrm{~cm}$, contains the LO of A. moronensis and was assigned to the lowermost portion of the A. moronensis Zone. However, because of the unreliable occurrence of $A$. moronensis, we placed this boundary at the LO of D. simonsenii in Sample 138-851B-25X-1, $80 \mathrm{~cm}$. The LO of C. coscinodiscus in Sample 138-851B-32HX-CC suggests that this sample is at least equivalent to or older than the $C$. coscinodiscus Zone. 


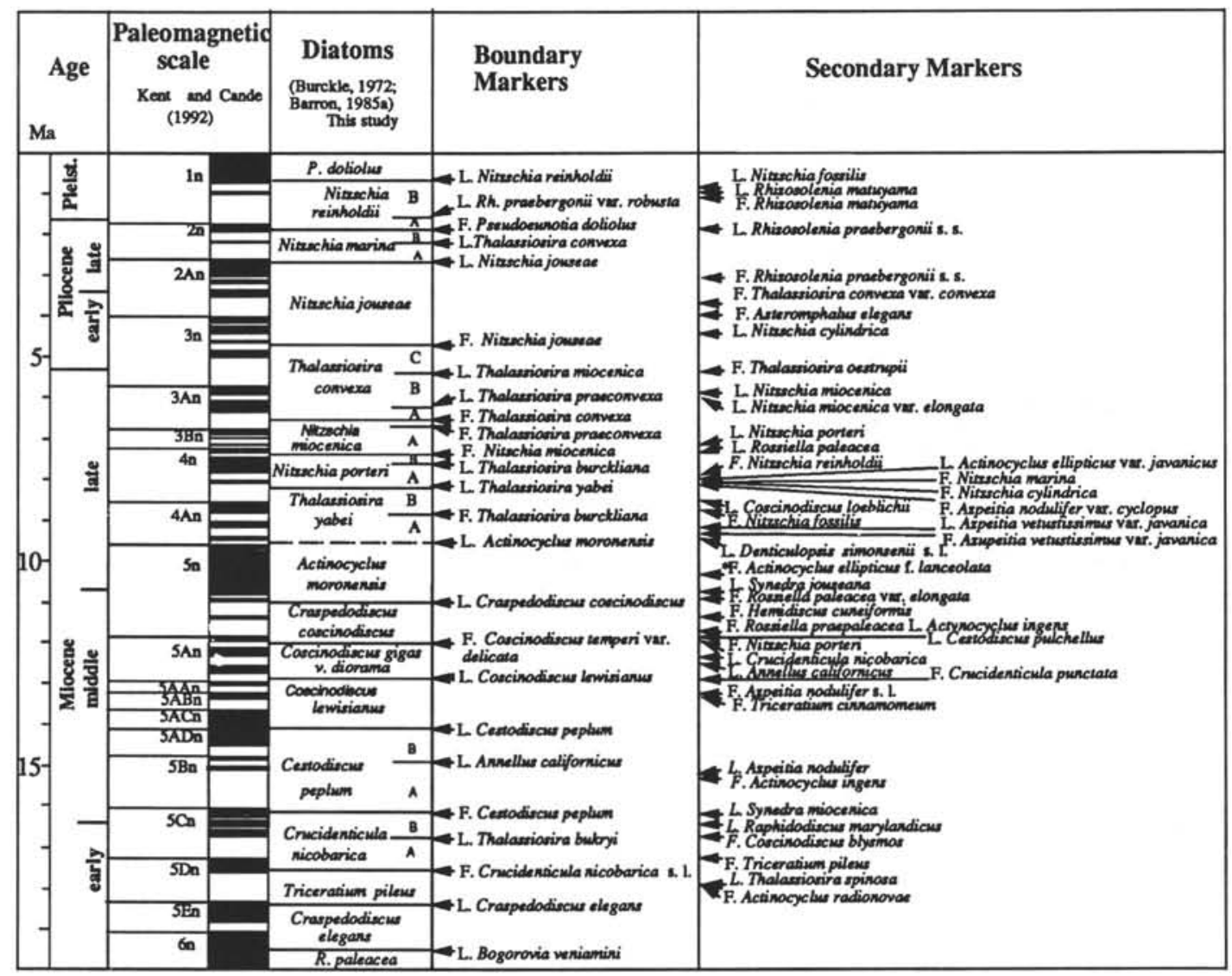

Figure 3. Correlation of primary and secondary stratigraphic markers used in this study. Events have been calibrated to the geochronology of Cande and Kent (1992). L = last occurrence, $\mathrm{F}=$ first occurrence.

\section{Site 852}

The stratigraphic sequence recovered consists of Pleistocene $(N$. reinholdii Zone) to uppermost middle Miocene ( $T$. yabei Zone) sediments. The shipboard biostratigraphy discussed in Mayer, Pisias, Janecek, et al. (1992) has been extensively revised as follows.

The LO of $N$. reinholdii is not well constrained at this site because of the scattered occurrence of this species in the uppermost portion of the stratigraphic sequence. The LO of Nitzschia fossilis in Sample $138-852 \mathrm{~B}-2 \mathrm{H}-2,25 \mathrm{~cm}$, and the occurrence of $R$. praebergonii in Sample 138-852B-3H-2, $25 \mathrm{~cm}$, indicates that these samples are equivalent to the $N$. reinholdii Zone. The FO of $P$. doliolus in Sample $138-852 \mathrm{~B}-3 \mathrm{H}-5,25 \mathrm{~cm}$, permits placement this sample in the $P$. doliolus Zone. The $N$. marina Zone extends from the FO of $P$. doliolus to the LO of $N$. jouseae. The interval from Samples 138-852B-3H-5, $25 \mathrm{~cm}$, to $-4 \mathrm{H}-2,25 \mathrm{~cm}$, was assigned to this zone. The FO of $N$. jouseae, which marks the base of the $N$. jouseae Zone, was placed in Sample 138-852B-6H-4, $120 \mathrm{~cm}$. Within this interval, the FO of $T$. convexa var. convexa was placed in Sample 138-852B-5H-2, $5 \mathrm{~cm}$, and the LO of $N$. cylindrica was placed in Sample 138-852B-6H-5, $120 \mathrm{~cm}$. The FO of $T$. convexa in Sample 138-852B-8H-6, $25 \mathrm{~cm}$, suggesting that this sample approximates the base of the $T$. convexa Zone. The LO of $N$. miocenica in Sample 138-852B-7H-4, $25 \mathrm{~cm}$, marks the $\mathrm{B} / \mathrm{C}$ subzonal boundary. The $\mathrm{FO}$ of $T$. praeconvexa in Sample $8 \mathrm{H}-2,25 \mathrm{~cm}$, marks the A/B subzonal boundary.

Samples $138-852 \mathrm{~B}-8 \mathrm{H}-6,25 \mathrm{~cm}$, to $-19 \mathrm{H}-2,25 \mathrm{~cm}$, represent the interval from the FO of $T$. convexa var. aspinosa to the FO of $N$. miocenica and were placed in the $N$. miocenica Zone. The FO of $T$. praeconvexa also in Sample 138-852B-8H-6, $25 \mathrm{~cm}$ (i.e., equivalent to the placement of the FO of $T$. convexa) results from both the sample interval and the low sedimentation rates at this site. The LO of T. yabei in Sample 138-852B-11 H-4, $25 \mathrm{~cm}$, allowed us to assign the interval from Samples 138-852B-9H-3, $75 \mathrm{~cm}$, through $-11 \mathrm{H}-4,25 \mathrm{~cm}$, to the $N$. porteri Zone. The subzonal A/B boundary was placed in Sample $138-852 \mathrm{~B}-11 \mathrm{H}-4,25 \mathrm{~cm}$. The FO of $N$. cylindrica occurs in Sample $138-852 \mathrm{~B}-10 \mathrm{H}-2,25 \mathrm{~cm}$. The occurrence of $T$. burckliana in Sample $138-852 \mathrm{~B}-11 \mathrm{H}-4,25 \mathrm{~cm}$, suggests that this sample approximates the lower portion of Subzone B of the T. yabei Zone.

\section{DISCUSSION}

The recovery of both a well-preserved diatom assemblage and paleomagnetic stratigraphies from Sites $844,845,848,851$, and 852 allowed us to evaluate the correlation of specific diatom events to the paleomagnetic record. The good quality magnetostratigraphy and reasonable sample spacing (generally less than $1.5 \mathrm{~m}$ ) allowed us to complete such correlations for about the last 6 m.y. Variability of the magnetostratigraphy and/or poor stratigraphic constraint limits such correlations for that part of the stratigraphic sequence older than 6 m.y. Correlations of specific diatom biostratigraphic events with the magnetostratigraphy of Schneider et al. (this volume) were completed for Sites 844 (Fig. 4), 845 (Figs. 5 and 6), Site 848 (Fig. 7), Site 851 (Fig. 8), and Site 852 (Fig. 9). Only those events that presently are considered reliable have been discussed below. The number of each event corresponds to that used in the tables.

1. LO Nitzschia reinholdii. This event has been widely used as a primary stratigraphic indicator for the low latitudes and to a lesser degree in the middle and high latitudes. The LO of $N$. reinholdii (i.e., $N$. reinholdii of Barron and Baldauf, 1986) in the low latitudes has 


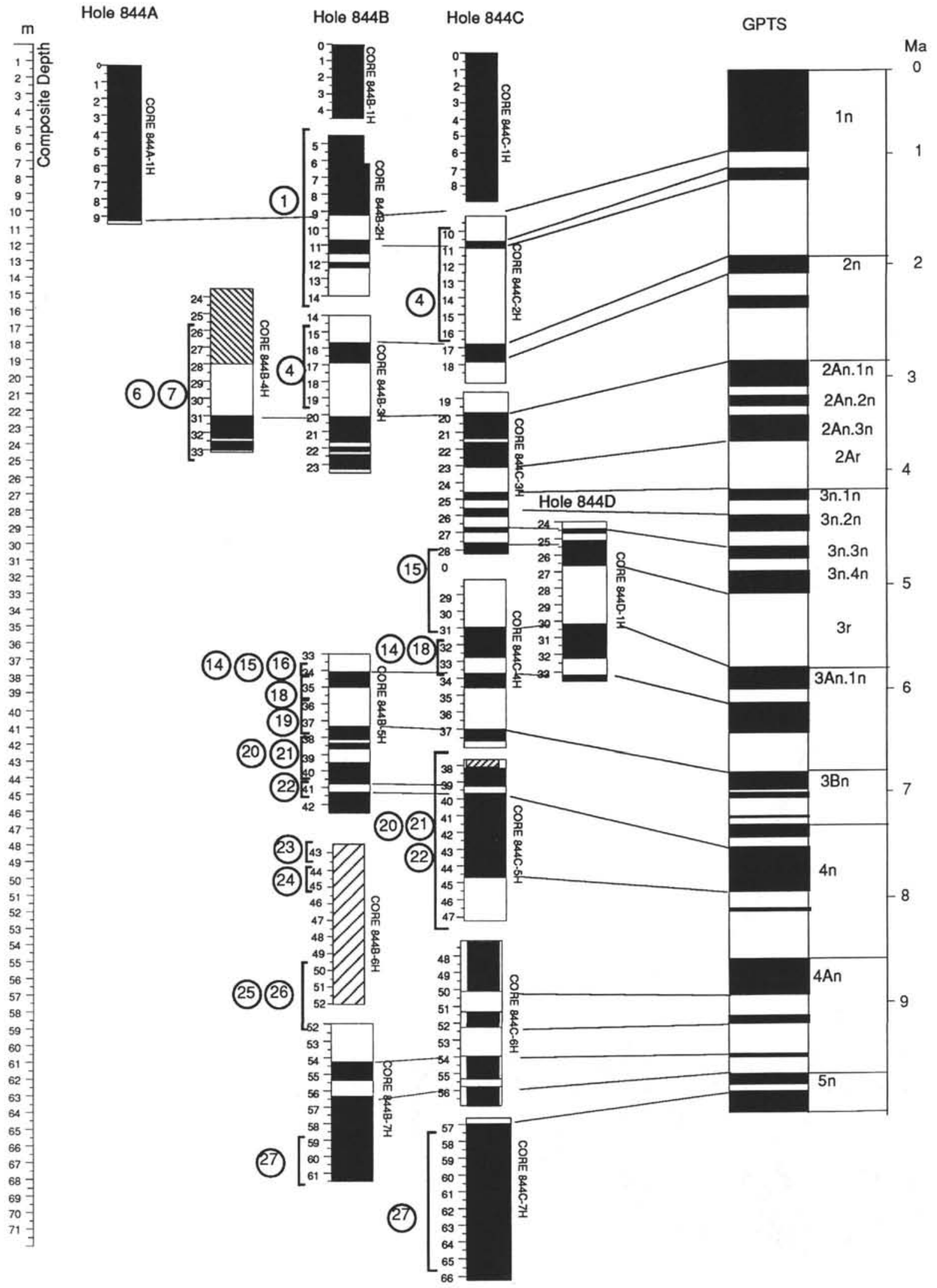

Figure 4. Diatom biostratigraphic events correlated with the paleomagnetic results (Schneider et al., this volume) for Site 844. Events are plotted both in mbsf and mcd. $1=\mathrm{LO}$ of $N$. reinholdii; $4=\mathrm{FO}$ of $P$. doliolus $; 6=\mathrm{LO}$ of $N$. jouseae $; 7=\mathrm{FO}$ of $R$. praebergonii; $14=\mathrm{LO}$ of $N$. miocenica $; 15=\mathrm{LO}$ of $N$. miocenica var. elongata. $16=\mathrm{LO}$ of $T$. praeconvexa $; 18=\mathrm{FO}$ of $T$. convexa var. aspinosa $; 19=\mathrm{FO}$ of $T$. praeconvexa $; 20=\mathrm{FO}$ of $N$. miocenica; $21=\mathrm{LO}$ of $R$. paleacea $; 22=\mathrm{FO}$ of $N$. reinholdii; $23=\mathrm{FO}$ of $N$. cylindrica; $24=\mathrm{LO}$ of $T . y a b e i$; $25=\mathrm{LO}$ of $C$. loeblichi; $26=\mathrm{FO}$ of $T$. burckliana $; 27=\mathrm{LO}$ of $D$. simonsenii. 


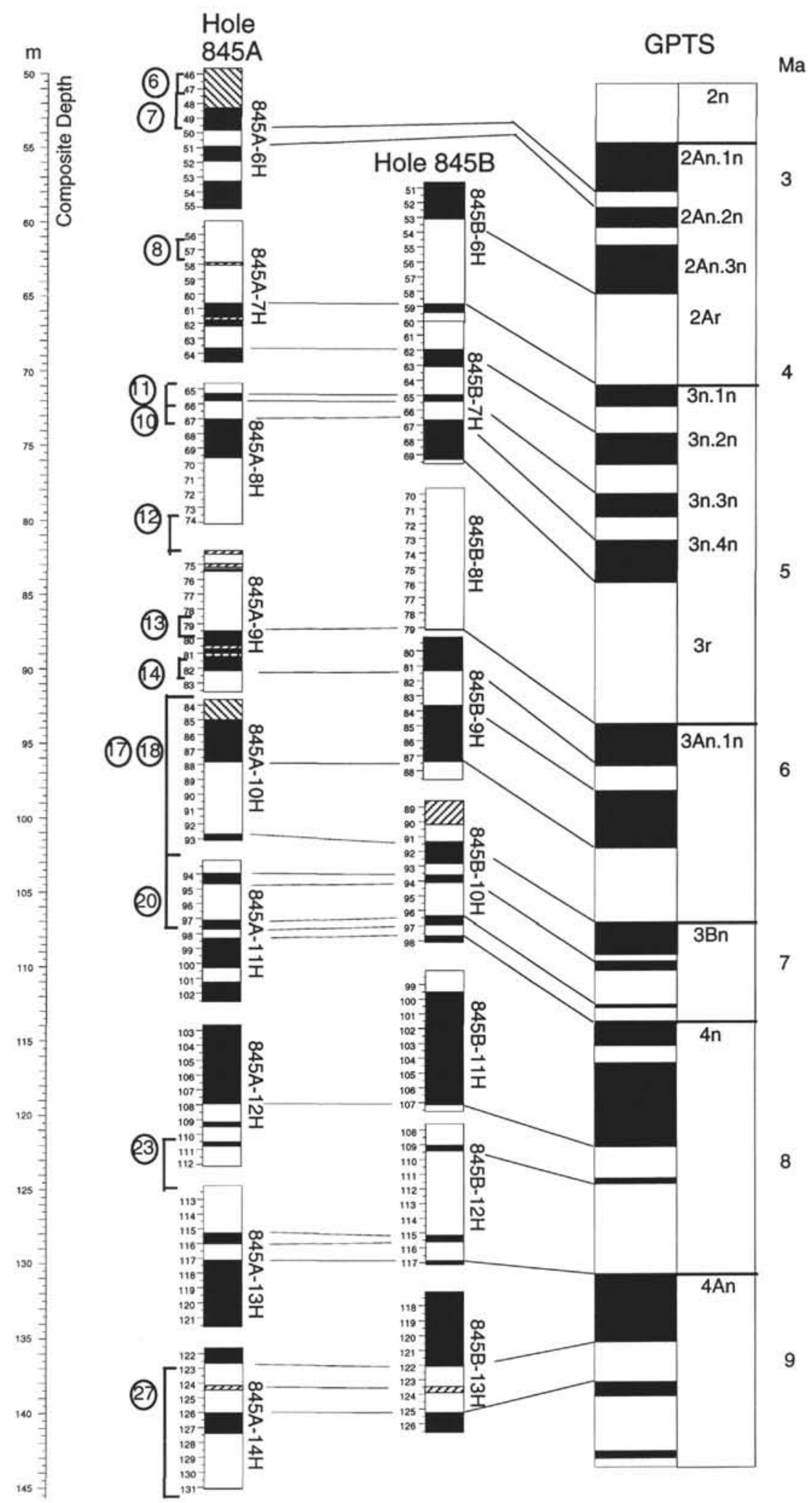

Figure 5. Diatom biostratigraphic events correlated with the paleomagnetic results (Schneider et al., this volume) for Site 845. Events are plotted both in mbsf and mcd. $6=\mathrm{LO}$ of $N$. jouseae; $7=\mathrm{FO}$ of $R$. praebergonii; $8=\mathrm{LO}$ of $T$. convexa $; 10=\mathrm{LO}$ of $N$. cylindrica $; 11=\mathrm{FO}$ of $N$. jouseae $; 12=$ FO of T. oestrupii; $13=\mathrm{LO}$ of $T$. miocenica; $14=\mathrm{LO}$ of $N$. miocenica $; 17=\mathrm{FO}$ of $T$. miocenica; $18=\mathrm{FO}$ of $T$. convexa var. aspinosa $; 20=\mathrm{FO}$ of $N$. miocenica $; 23=\mathrm{FO}$ of $N$. cylindrica $; 27=\mathrm{LO}$ of $D$. simonsenii; $28=\mathrm{LO}$ of $A$. ingens: $29=\mathrm{LO}$ of $C$. lewisianus. 


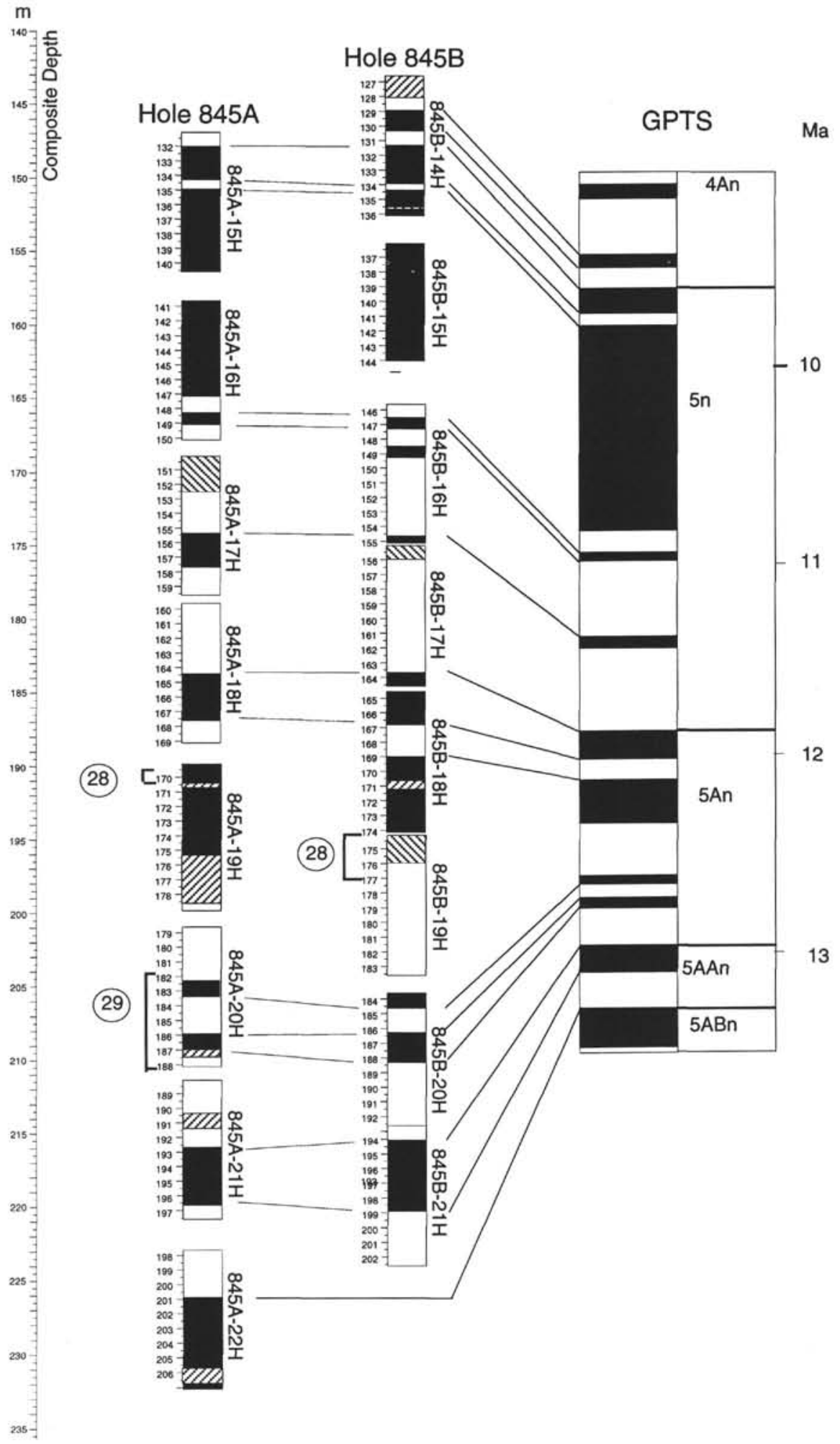

Figure 5 (continued).

been correlated by Burckle (1977, 1978), Barron (1985a, 1985b), and Baldauf (1985; among other researchers) with the lowermost portion of the Brunhes Chron (Cln of Cande and Kent, 1992) and has an estimated age of 0.65 Ma (also 0.65 Ma using Cande and Kent, 1992; see Table 2). In the middle latitude Pacific (Koizumi and Tanimura, 1985), North Atlantic (Baldauf, 1984 and 1987), and the Indian
Ocean (Schrader, 1974), the LO of $N$. reinholdii has been calibrated to the middle to upper portion of the Brunhes Chron (C1n of Cande and Kent, 1992). However, Mikkelsen (1990) correlated the LO of $N$. reinholdii with the uppermost part of the Matuyama Chron (C1n.1n) approximately and assigned an estimated age of $0.9 \mathrm{Ma}$ (1.0 Ma using Cande and Kent, 1992). This approximates the last continuous occur- 


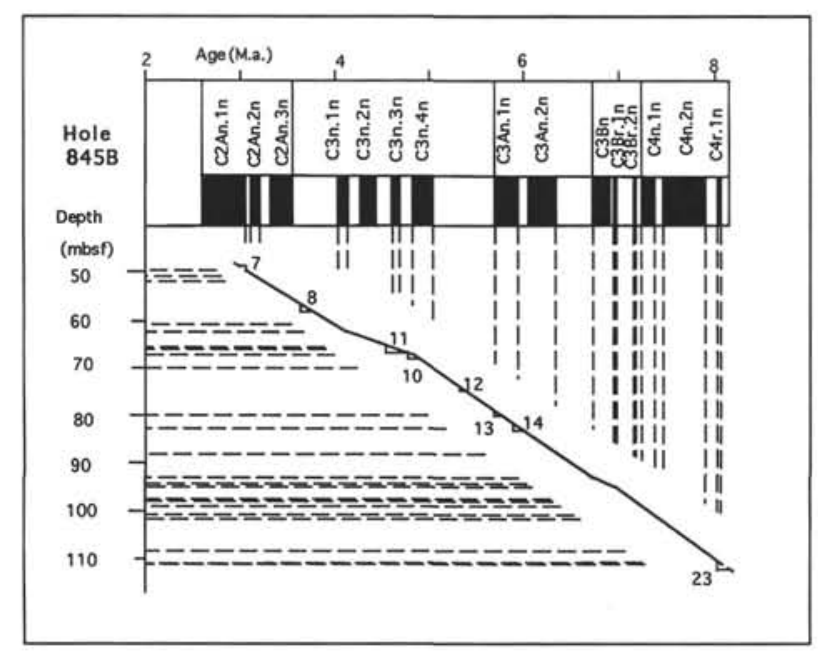

Figure 6. Calibration of selected events to the magnetostratigraphic results of Schneider (this volume) for Hole 845B. Event: $7=\mathrm{FO}$ of $R$. praebergonii; 8 $=\mathrm{LO}$ of $T$. convexa $; 11=\mathrm{FO}$ of $N$. jouseae $; 10=\mathrm{LO}$ of $N$. cylindrica $; 12=$ LO of T. oestrupii group; $13=\mathrm{LO}$ of T. miocenica; $14=\mathrm{LO}$ of N. miocenica; $23=\mathrm{FO}$ of $N$. cylindrica.

rence of $N$. reinholdii at several of the Leg 138 sites. Age estimates for this event by these authors range between 0.44 and $0.9 \mathrm{Ma}(0.4$ 1.0 Ma using Cande and Kent, 1992).

The stratigraphic range of $N$. reinholdii is sometimes scattered in the upper half of its range in the Leg 138 sediments. This is especially true at Sites 844,849 , and 850 . This event is well constrained in Holes $845 \mathrm{~A}, 846 \mathrm{~B}, 851 \mathrm{~B}$, and $851 \mathrm{E}$. At these latter sites, a reliable magnetostratigraphy occurs only at Site 851 . In both Holes $851 \mathrm{~B}$ and $851 \mathrm{E}$, the LO of $N$. reinholdii correlates with the lower portion of Cln (Fig. 8) and has an age estimate of between 0.46 and $0.57 \mathrm{Ma}$ in Hole $851 \mathrm{~B}$ and an age of between 0.66 and $0.69 \mathrm{Ma}$ in Hole 851E.

2. LO Nitzschia fossilis. This event has been noted by numerous researchers to approximate the LO of the silicoflagellate Mesocena quadrangula (Barron, 1985a, 1985b; Baldauf, 1984, 1985). A similar correspondence can be seen at the Leg 138 sites. The LO of $N$. fossilis has been correlated by Barron (1980) to approximate the top of the Jaramillo Subchron (equivalent to $\mathrm{C} \ln .1 \mathrm{n}$ ) with an approximate age of $0.85 \mathrm{Ma}$ (0.92 Ma using Cande and Kent, 1992). At ODP Site 710 in the Indian Ocean, Mikkelsen (1990) reported that the LO of $N$. fossilis lies in the upper part of the Matuyama Chron. In the northwest Pacific (Koizumi and Tanimura, 1985) and in the North Atlantic (Baldauf, 1984), this event correlates with the lowermost portion of the Brunhes Chron (equivalent to $\mathrm{Cln}$ ), indicating that this event is diachronous between various oceanographic regions.

The LO of $N$. fossilis is constrained in Holes $845 \mathrm{~B}, 846 \mathrm{~B}, 851 \mathrm{C}$, and $851 \mathrm{E}$. The reliable magnetostratigraphy in holes from Site 851 (Fig. 8) allowed for direct comparisons between this event and the magnetostratigraphy. The LO of $N$. fossilis correlates with the lowermost portion of $\mathrm{C} \ln$ to the upper portion of $\mathrm{C} \ln \ln$ (midway between $\mathrm{C} 1 \mathrm{n}$ and $\mathrm{C} \ln .1 \mathrm{n}$ ). This event has an estimated age of between 0.78 and $0.85 \mathrm{Ma}$ in Hole $851 \mathrm{C}$ and an age of between 0.68 and $0.88 \mathrm{Ma}$ in Hole $851 \mathrm{E}$. This event clearly is useful as a secondary indicator in the eastern equatorial Pacific.

3. LO Rhizosolenia praebergonii. The LO of $R$. praebergonii was correlated by Burckle and Trainer (1979) and Barron (1985a) with an interval just above the Olduvai Subchron (equivalent to $C 2 n$ ) having an estimated age of 1.55 Ma (1.64 Ma using Cande and Kent, 1992). Koizumi and Tanimura (1985) correlated this event with the lower portion of the Matuyama Chron and assigned an age of 2.4 to $2.5 \mathrm{Ma}$. Baldauf (1984) indicated that $R$. praebergonii had a scattered strati- graphic occurrence in the North Atlantic, suggesting that this event is stratigraphically useful only regionally.

The LO of $R$. praebergonii is well constrained in Holes 846B, 847B, 847C, 849B, 849C, 851B, and 852B. Paleomagnetic control for these holes is well constrained in Holes 852B (Fig. 9). In Hole $852 \mathrm{~B}$, the $\mathrm{LO}$ of $R$. preabergonii approximates the top of $\mathrm{C} 2 \mathrm{n}$ and has an estimated age of between 1.64 and $1.75 \mathrm{Ma}$. This age estimate is similar to those previously determined for this event in the eastern equatorial Pacific.

4. FO Pseudoeunotia doliolus. This event in the low latitude Pacific has been calibrated with the middle of the Olduvai Subchron (C2n) by Burckle $(1972,1977)$ and Barron (1985a). An age of 1.80 Ma (1.90 Ma using Cande and Kent, 1992) was assigned to this event by these authors. Several previous authors also have indicated that this event may be diachronous between the low and middle latitudes, with age estimates that range between 1.8 and $2.0 \mathrm{Ma}(1.9-2.1 \mathrm{Ma}$ using Cande and Kent, 1992) in the northwest Pacific (Koizumi and Tanimura, 1985).

The FO of $P$. doliolus is well constrained at Sites 848,851 , and 852 , where this event correlates to an interval directly above Chron C2An.1n (Figs. 7, 8, and 9). The FO of $P$. doliolus has an age of between 2.15 and $2.21 \mathrm{Ma}$ at Site 851 and an age of between 2.12 and $2.24 \mathrm{Ma}$ at Site 852. The age of 1.95 to $2.02 \mathrm{Ma}$ for this event at Site 848 is less reliable because of uncertainty in the placement of the magnetic events.

5. LO Thalassiosira convexa. Burckle (1978) calibrated this event to the lower portion of the Matuyama Subchron (equivalent to lower portion of $\mathrm{C} 2 \mathrm{n}$ ) and assigned an age of $2.10 \mathrm{Ma}$ (using Cande and Kent, this calibration has an age of $2.21 \mathrm{Ma}$ ) for this event in the equatorial Pacific. An equivalent age was determined by Baldauf (1985) and Barron (1985a) also for the equatorial Pacific region. This event has been best constrained at Site 851 (Fig. 9), where it correlates to an interval directly above Chron $2 \mathrm{An}$. $1 \mathrm{n}$. At this site, this event has an estimated age of $2.35 \mathrm{Ma}$. Note that this event also correlates to the magnetostratigraphy at Site 844 (Fig. 4). However, the sampling constraint at this site is coarser. At Site 844 , this event is older and occurs in $\mathrm{C} 2 \mathrm{An} .1 \mathrm{n}$ through $\mathrm{C} 3 \mathrm{n} .3 \mathrm{n}$. This older correlation reflects an inconsistent occurrence of the species at Site 844 . This event also was recognized at Site 848 (Fig. 7), but a poor magnetic record precluded age estimates.

6. LO Nitzschia jouseae. This event is well constrained at Sites 851 (Fig. 8) and 852 (Fig. 9). At Site 851, this event correlates to the middle portion of C2An. $1 \mathrm{n}$ and has an age of 2.75 to 2.80 Ma. At Site 852, this event correlates with the uppermost portion of C2An.In and has an estimated age of 2.60 to $2.75 \mathrm{Ma}$. This slightly younger age at Site 852 may reflect misplacement of the top of $\mathrm{C} 2 \mathrm{An}$. In as a result of a coring gap (see Fig. 9). The ages of this event derived from this study approximate those from previous studies. Koizumi and Tanimura (1985) assigned an age of 2.48 to $2.58 \mathrm{Ma}$ (equivalent to about 2.6-2.7 Ma using Cande and Kent, 1992) to this event. Likewise, Barron (1985a) assigned an age of 2.6 Ma (2.73 Ma using Cande and Kent, 1992).

7. FO Rhizosolenia praebergonii. The FO of this species is well constrained at Sites 851 and 852 . At these sites, this event correlates with an interval representing the lowermost portion of $\mathrm{C} 2 \mathrm{An}$. In to the uppermost portion of C2An.2n. The estimated age of this event is 3.0 to $3.15 \mathrm{Ma}$. This age in comparable to that of Burckle (1978) and Barron (1985a) for the equatorial Pacific region. Koizumi and Tanimura (1985) indicated that $R$. praebergonii has a shorter duration at the mid-latitude Pacific (DSDP Hole 580) of about 300 k.y. compared to its longer duration (about $1.5 \mathrm{Ma}$ occurrence) in the lower latitudes. The brief occurrence of $R$. praebergonii was interpreted by Koizumi and Tanimura (1985) to represent incursion of this species into the middle latitudes during warm events. This was also implied by its scattered occurrence in the high latitudes of the North Atlantic during the latest Pliocene (see Baldauf, 1984, 1987). This event is well constrained at Site 848 (Fig. 7). Here, the FO of $R$. praebergonii 


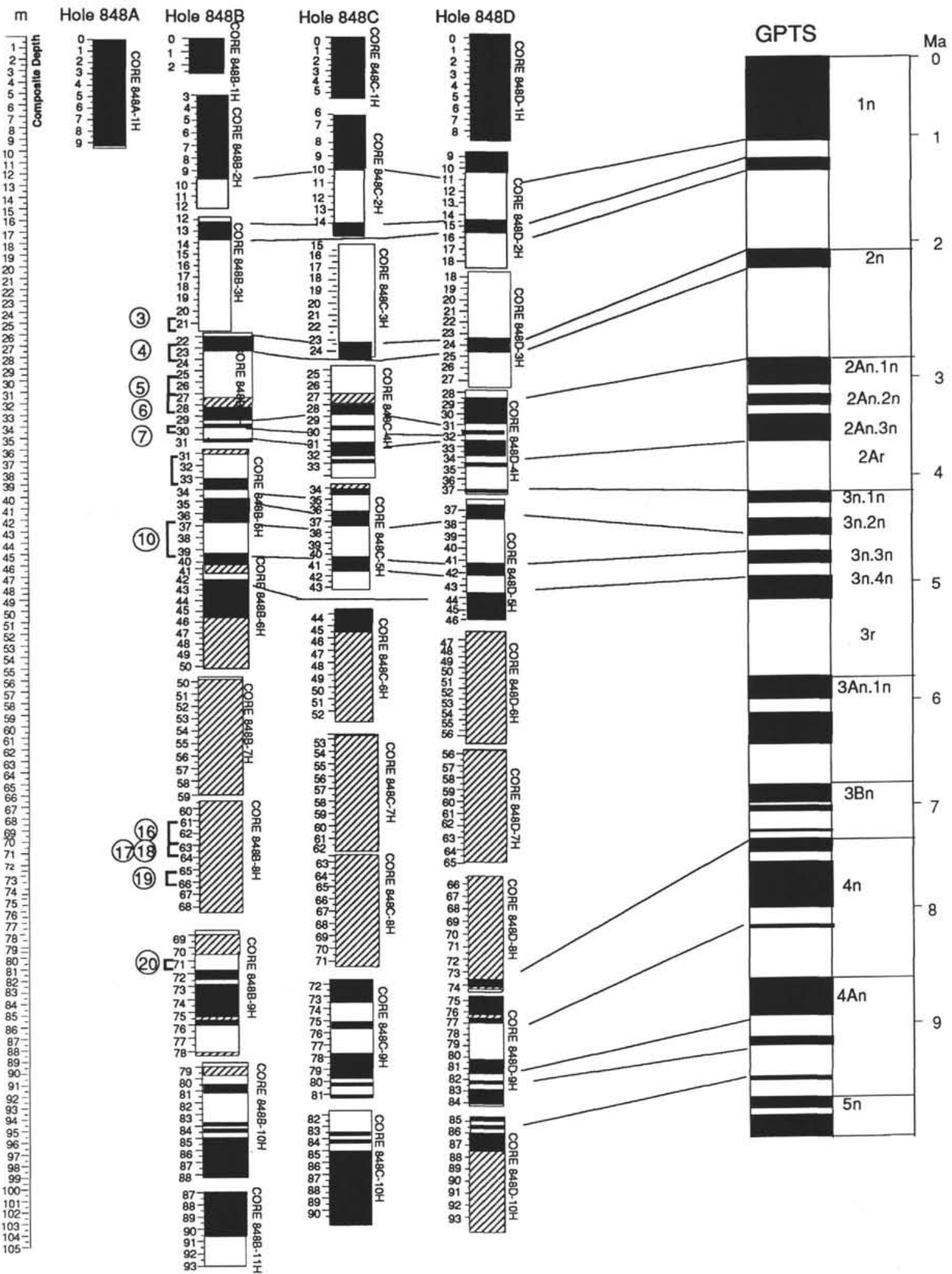

Figure 7. Diatom biostratigraphic events correlated with the paleomagnetic results (Schneider et al., this volume) for Site 848 . Events have been plotted both in mbsf and mcd. $3=\mathrm{LO}$ of $R$. praebergonii; $4=\mathrm{FO}$ of $P$. doliolus; $5=\mathrm{LO}$ of $T$. convexa $\mathrm{s} .1 . ; 6=\mathrm{LO}$ of $N$. jouseae $; 7=$ FO of $R$. praebergonii $; 10=\mathrm{LO}$ of $N$. cylindrica $; 16=\mathrm{LO}$ of $T$. praeconvexa $; 17=\mathrm{FO}$ of $T$. miocenica $; 18=\mathrm{FO}$ of $T$. convexa var. aspinosa; $19=\mathrm{FO}$ of $T$. praeconvexa $; 20=\mathrm{FO}$ of $N$. miocenica. 


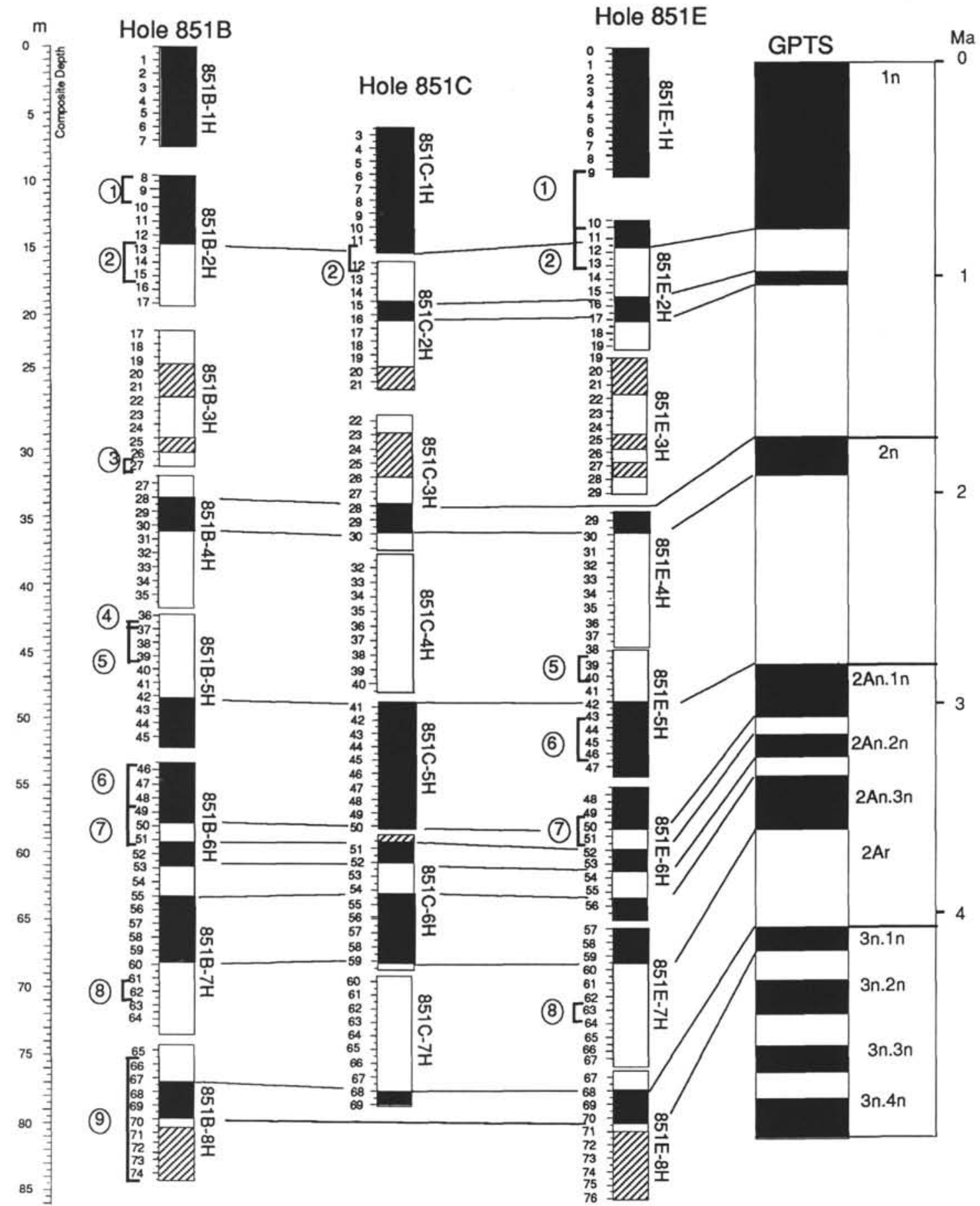

Figure 8. Diatom biostratigraphic events correlated with the paleomagnetic results (Schneider et al., this volume) for Site 851. Events were plotted both in mbsf and mcd. $1=\mathrm{LO}$ of $N$. reinholdii; $2=\mathrm{LO}$ of $N$. fossilis; $3=\mathrm{LO}$ of $R$. praebergonii; $4=\mathrm{FO}$ of $P$. doliolus; $5=\mathrm{LO}$ of $T$. convexa s.1.; $6=\mathrm{LO}$ of $N$. jouseae $; 7=\mathrm{FO}$ of $R$. praebergonii $; 8=\mathrm{LO}$ of $T$. convexa $;=\mathrm{LO}$ of $A$. elegans.

approximates the upper portion of the interval between $\mathrm{C} 3 \mathrm{n} . \mathrm{ln}$ and $\mathrm{C} 3 \mathrm{n} .2 \mathrm{n}$. An age of $4.2 \mathrm{Ma}$ was estimated for this event at this site.

8. FO Thalassiosira convexa var. convexa. The FO of this variety is well documented for use in the eastern equatorial Pacific (see Burckle, 1978; Burckle and Trainer, 1979; Barron, 1985a; and Baldauf, 1985) and equatorial Indian Ocean (Schrader, 1974). This event was calibrated to the upper Gilbert (lower C2An-upper C3n) by Burckle (1978). An age estimate of 3.6 Ma (3.75 Ma, using Cande and Kent, 1992) was determined by Burckle and Trainer (1979) and Barron (1985a).
The FO of T. convexa var. convexa is well constrained stratigraphically at numerous Leg 138 sites (Sites 846 through 851). Unfortunately, direct correlation with a reliable magnetostratigraphy occurs in Holes 845A (Figs. 5 and 6) and 852B (Fig. 9). In Hole 845A, this event correlates with the middle to upper portion of $\mathrm{C} 2 \mathrm{An}$ and has an estimated age 3.64 to $3.79 \mathrm{Ma}$. The correlation of this event with the magnetostratigraphy in Hole $852 \mathrm{~B}$ is less constrained. In Hole $852 \mathrm{~B}$, this event correlates with most of the interval representing $\mathrm{C} 2 \mathrm{An}$. The age of this event in Hole $852 \mathrm{~B}$ is between 3.63 to $3.88 \mathrm{Ma}$. These age constraints are in agreement with the estimated age of 3.6 


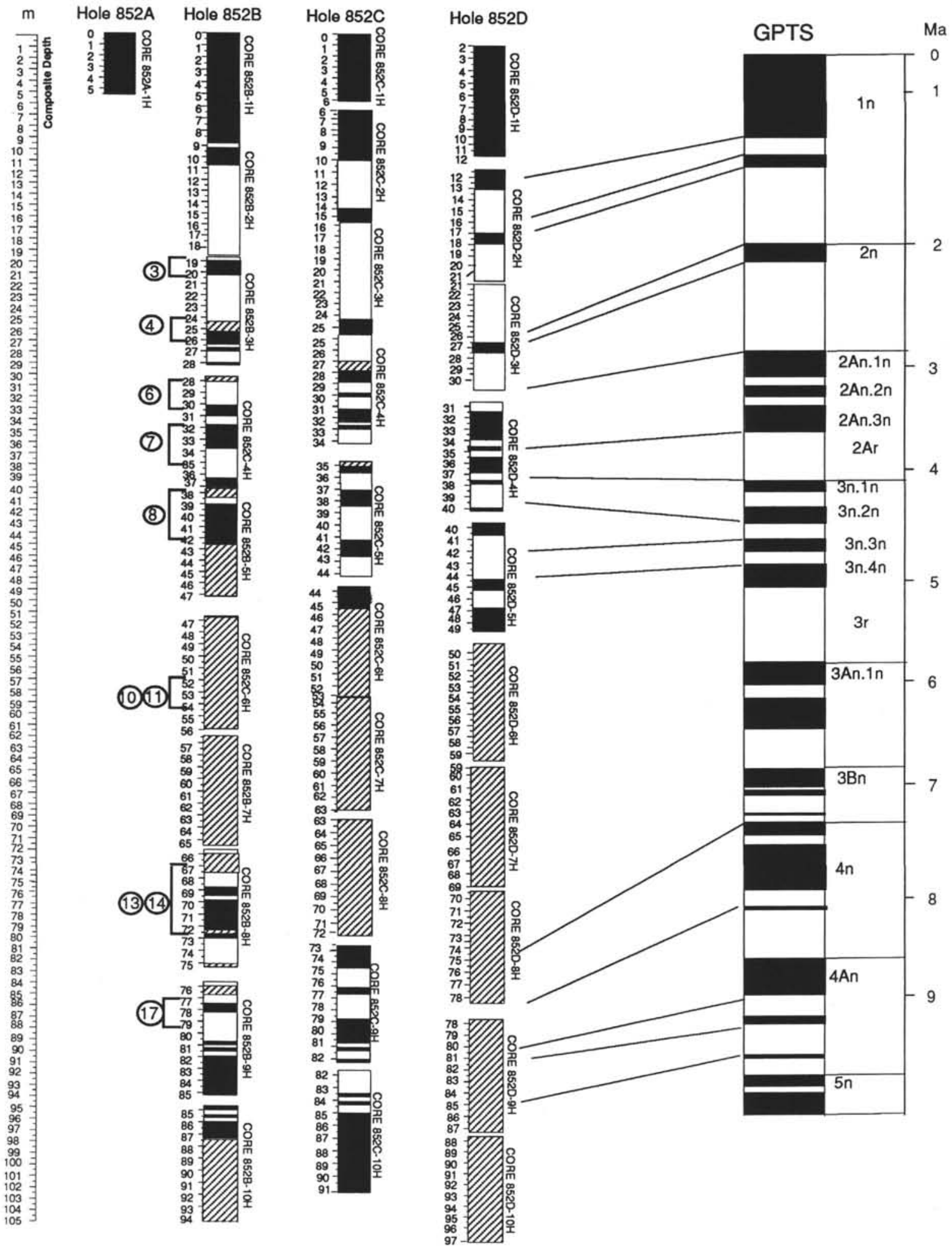

Figure 9. Diatom biostratigraphic events correlated with the paleomagnetic results (Schneider et al., this volume) for Site 852. Events were plotted in both mbsf and mcd. $3=\mathrm{LO}$ of $R$. praebergoni; $4=\mathrm{FO}$ of $P$. doliolus; $6=\mathrm{LO}$ of $N$. jouseae $; 7=\mathrm{FO}$ of $R$. praebergonii; $8=\mathrm{LO}$ of T. convexa $; 10=\mathrm{LO}$ of $N$. cylindrica $; 11=\mathrm{FO}$ of $N$. jouseae $; 13=\mathrm{LO}$ of $T$. miocenica $; 14=\mathrm{LO}$ of $N$. miocenica $; 17=\mathrm{FO}$ of $T$. miocenica. 
Ma (3.75 Ma using Cande and Kent, 1992), assigned by Burckle and Trainer (1979) and Barron (1985a).

9. FO Asteromphalus elegans. Although this species occurs throughout the low latitudes of the world oceans, the stratigraphic occurrence of this species is poorly constrained. The stratigraphic continuity of this event is best known in the eastern equatorial Pacific (Burckle, 1978; Barron, 1985a, 1985b). In this region, Burckle (1978) calibrated this event to event a' of the Gilbert Chron (C3n.1n) and assigned an estimated age of 3.9 Ma (4.06 Ma, using Cande and Kent, 1992). Baldauf $(1984,1987)$ noted the occurrence of this species in the middle latitude North Atlantic, but noted that this species had a scattered and inconsistent stratigraphic occurrence.

The occurrence of this species in the Leg 138 sediments is scattered, resulting in this event being reasonably constrained only in Holes $848 \mathrm{~B}$ and $851 \mathrm{C}$. However, the paleomagnetic stratigraphy for these stratigraphic intervals is unreliable (see Fig. 7). In Hole 851C, the FO of Asteromphalus elegans approximates $\mathrm{C} 3 \mathrm{n} .1 \mathrm{n}$, suggesting an age somewhat similar to that determined by Burckle (1978), but additional samples are necessary to constrain this event further.

10. LO Nitzschia cylindrica. The LO of $N$. cylindrica is a useful secondary stratigraphic indicator for the equatorial Pacific (Burckle, 1978; Barron, 1985a, 1985b), the low to middle latitudes of the North Atlantic (Baldauf, 1984, 1987; Baldauf and Pokras, 1990), and the low latitudes of the Indian Ocean (Schrader, 1974). Burckle (1978) calibrated this event to event c' of the Gilbert Chron (C3n.3n) in the eastern equatorial Pacific. Both Burckle (1978) and Barron (1985a) assigned an age of $4.3 \mathrm{Ma}$ (4.5 Ma, using Cande and Kent, 1992) to this event in the eastern equatorial Pacific.

The LO of $N$. cylindrica is constrained in the Leg 138 sediments at Sites 846 through 849,851 , and 852 . Calibration of the Leg 138 magnetostratigraphy is completed in Holes $845 \mathrm{~B}$ (Fig. 5), 848B (Fig. 7), and $852 \mathrm{~B}$ (Fig. 9). However, the magnetostratigraphy is somewhat in question for Holes $848 \mathrm{~B}$ and $852 \mathrm{~B}$. In Hole $845 \mathrm{~A}$, this event approximates the interval from the base of $\mathrm{C} 3 n .3 n$ to the uppermost portion of $\mathrm{C} 3 \mathrm{n} .4 \mathrm{n}$. This event has an estimated age at this site of between 4.48 and $4.75 \mathrm{Ma}$. In Hole $852 \mathrm{~B}$, this event correlates with the uppermost portion of $\mathrm{C} 3 \mathrm{n} .4 \mathrm{n}$ and has an tentative age of 4.82 to 4.9 Ma, slightly older than that derived by Burckle (1978). In Hole $848 \mathrm{~B}$, this event has a correlation similar to that in Hole $852 \mathrm{~B}$. However, the paleomagnetic stratigraphy above and below this event is poorly defined making these latter correlations questionable.

11. FO Nitzschia jouseae. This event is well documented for use through the warm water sphere, including the equatorial Pacific (Burckle, 1972 1977, 1978; Barron, 1985a, 1985b; Baldauf, 1985; Sancetta, 1984). In the middle latitudes of the North Atlantic, this event was recognized and determined to be stratigraphically useful by Baldauf $(1984,1987)$. Baldauf and Pokras (1990) also recognized the usefulness of this event in the low-latitude eastern Atlantic. The LO of $N$. jouseae has also been used as a zonal indicator in the Indian Ocean (Schrader, 1974). Burckle (1978) calibrated this event to the c' event of the Gilbert (C3n.3n) and assigned it an age of $4.5 \mathrm{Ma}(4.73$ Ma, using Cande and Kent, 1992).

This event has been stratigraphically constrained at Sites 844 through 850 and Sites 851 and 852 . Paleomagnetic stratigraphy allows correlation of this event to the magnetostratigraphy (Schneider et al., this volume) for Holes $845 \mathrm{~B}$ (Fig. 5) and 852B (Fig. 9). The FO of $N$. jouseae approximates the LO of $N$. cylindrica in both of these holes. In Hole 852B, the sample constraints are identical for both of these events. In Hole 845B, the FO of $N$. jouseae and the LO of $N$. cylindrica are observed in the same sample (138-845B-8H-2, $27 \mathrm{~cm})$. The only difference is that the other constraining samples occur over a 2-m interval.

This event approximates the upper portion of C3n.4n at Site 852 and occurs in an interval associated with C $3 n .3 n-C 3 n .4 n$ at Site 845. Age estimates for this event range between 4.75 and 4.82 and between
4.56 and $4.7 \mathrm{Ma}$. These ages approximate the earlier calibration of Burckle (1978), who estimated an age of 4.5 Ma (4.75 Ma, using Cande and Kent) for this event.

12. FO Thalassiosira oestrupii group. Burckle (1978), Barron (1985a), and Baldauf (1985) discussed the stratigraphic usefulness of this event as a secondary stratigraphic marker for the eastern equatorial Pacific. Barron (1981) actually defined a Thalassiosira oestrupii Zone with the base of this zone defined by the FO of $T$. oestrupii. This zone was used for the middle latitude Pacific. A somewhat similar zone was developed for use in the southern ocean by Baldauf and Barron (1991). T. oestrupii also has been shown to be stratigraphically useful in the Indian Ocean (Schrader 1974) and the North Atlantic (Baldauf 1984, 1987; Baldauf and Pokras, 1990).

The FO of $T$. oestrupii has been calibrated to an interval just above Chron 5 (C3An) by Burckle (1978). Barron (1981) suggested that this event approximated the lowermost reversed interval of the Gilbert Chron (lower C3n) and had estimated an age of 5.1 Ma (5.42 Ma, using Cande and Kent, 1992). In the southern ocean, Baldauf and Barron (1991) also assigned an age of 5.1 Ma (5.42 Ma, using Cande and Kent, 1992) for this event. Note that Bodén (1992) recently completed a detailed taxonomic analysis of several forms of Thalassiosira. In doing so, he defined several new species, some of which were most likely grouped into $T$. oestrupii by previous researchers. In the Leg 138 sediments, we grouped various forms of Thalassiosira into the $T$. oestrupii group.

The FO of $T$, oestrupii group was observed in Holes 845A, 846B, and $846 \mathrm{D}$. The paleomagnetics for Hole $845 \mathrm{~A}$ suggest that the FO of this event approximates the middle portion of $\mathrm{C} 3 \mathrm{n}$ and has an age of between 5.38 and $5.46 \mathrm{Ma}$. This age estimate is tentative, as this event occurs over an interval that represents a coring gap.

13. LO Thalassiosira miocenica. This event was used as a secondary stratigraphic indicator in the eastern equatorial Pacific by Burckle (1972, 1977, 1978), Barron (1985a, 1985b), and Baldauf (1985). This event also was recognized as being useful in the middle latitude North Atlantic (Baldauf 1984, 1987) and Indian Ocean (Schrader, 1974). The LO of T. miocenica approximates the FO of $T$. oestrupii and the $T$. oestrupii group and was calibrated to the lowermost Gilbert Chron (C3n) by Burckle (1978). This event has an estimated age of 5.1 Ma (5.42 Ma, using Cande and Kent, 1992).

The LO of $T$. miocenica is recorded and well constrained at Sites 845 through $847,849,850$, and 852 . Hole 845 B (Fig. 5) has the most reliable magnetostratigraphy for correlation. At this site, this event was calibrated with the lowermost portion of $\mathrm{C} 3 n$ to the uppermost portion of C3An. $\mathrm{n}$ in this hole and has an age of between 5.63 and $5.73 \mathrm{Ma}$. The slightly older age for this event compared to that of Burckle (1978) may result from the constraints of the paleomagnetic stratigraphy in the upper portion of Core 138-845B-9H (see Fig. 6).

14. LO Nitzschia miocenica. Similar to most of the other events discussed above, the LO of $N$. miocenica has been used as a stratigraphic marker in the low-latitude Pacific, the low-to middle-latitude North Atlantic, and the low-latitude Indian Ocean (Burckle, 1972, 1977, 1978; Barron, 1985a, 1985b; Baldauf, 1984, 1985, 1987; Schrader, 1974). In addition, this species was observed in the extreme southern latitudes of the Indian Ocean in the Kerguelen Plateau region and appeared to have a similar stratigraphic range as those determined elsewhere (Baldauf and Barron, 1991). In the eastern equatorial Pacific, Baldauf (1985) quantitatively determined that the last abundant occurrence of this species was at $5.6 \mathrm{Ma}(6.00 \mathrm{Ma}$, using Cande and Kent, 1992), about 0.1 Ma prior to the LO of this species.

Although this event has been determined at Sites 844 through 846, 849,850 , and 852 , the combined biostratigraphy and magnetostratigraphy can be correlated in Holes $845 \mathrm{~B}$ and $852 \mathrm{~B}$. In Hole $845 \mathrm{~B}$, this event correlates with the lowermost portion of $\mathrm{C} 3 \mathrm{An}$. In and the uppermost portion of the reversed interval between C3An. $1 n$ and C $3 A n .2 n$. An age estimate cannot be calculated as the base of C $3 \mathrm{An} .2 \mathrm{n}$ is uncer- 
tain in this hole. The LO of $N$. miocenica has a similar correlation in Hole $852 \mathrm{~B}$; however, the placement of the base of C $3 \mathrm{An} .2 \mathrm{n}$ also is uncertain in this hole.

Stratigraphic events that occur in sediments older than those that contain the LO of N. miocenica are less tightly constrained either by the sampling interval, preservation, or by the uncertainty in the magnetostratigraphic record. As such, these are not discussed here.

16. LO Thalassiosira praeconvexa. This event marks the Subzone A/B boundary of the Thalassiosira convexa Zone, as used by Burckle (1977), Barron (1985a, 1985b), and Baldauf and Iwai (this study). This event has been recognized throughout the low-latitude Pacific (Barron, 1985a, 1985b) the low- and middle-latitude North Atlantic (Baldauf, 1984, 1987), and the southern portion of the Indian Ocean (Baldauf and Barron, 1991). Burckle (1978) calibrated this event to paleomagnetic Chron C3An.2n and assigned an age of 5.8 Ma (6.25 Ma, using Cande and Kent, 1992).

This event was identified at Leg 138 Sites 844 and 846 through 851 and has been calibrated at Site 844 to an interval equivalent to the uppermost portion of paleomagnetic Chron $\mathrm{C} 3 \mathrm{An} .2 \mathrm{n}$ to the lowermost portion of C3An.1n (Fig. 3). Based on this correlation, this event has an estimated age of 6.15 Ma at Site 844 (Fig. 3).

18. FO Thalassiosira convexa. Burckle (1972), Barron (1985a, $1985 \mathrm{~b})$, and Baldauf $(1984,1987)$, among others, have used this event to mark the base of the T. convexa Zone. Although Schrader (1974) recorded $T$. convexa in samples from DSDP Site 238 in the Indian Ocean, Schrader preferred to use the FO of $T$. miocenica to mark the base of his Zone 15. Barron (1985a, 1985b) and Baldauf (1984, 1987) indicated that the FO of $T$. miocenica and T. convexa var. aspinos $a$ are contemporaneous, and any difference in the stratigraphic placement of these events is a result of difference in species concepts.

Burckle (1978) calibrated the FO of $T$. convexa to paleomagnetic Chron C3An. $2 \mathrm{n}$ and assigned an age of $6.1 \mathrm{Ma}$ (6.54 Ma, using Cande and Kent, 1992). This event is recorded at Sites 844 through 846 and 848 through 851. At Site 844, this event correlates with the interval equivalent to the lower portion of paleomagnetic event $\mathrm{C} 3 \mathrm{An} .2 \mathrm{n}$ to the upper portion of C3An.2n. An estimated age of 6.15 to $6.42 \mathrm{Ma}$ was calculated for this event at Site 844 , based on sampling constraints.

20. FO Nitzschia miocenica. Burckle (1972, 1977), Barron (1985a, 1985b), and Baldauf and Iwai (this study) used this event to mark the base of the $N$. miocenica Zone. This event also is stratigraphically useful in the low-latitude Indian Ocean and has been used to mark the base of Zone 17 (Schrader, 1974). This event was not recognized in the middle- to high-latitude North Atlantic as N. miocenica has a scattered occurrence in this region (Baldauf, 1984, 1987). Barron (1992) calibrated this event to paleomagnetic event $\mathrm{C} 4 \mathrm{n}$. In and assigned an estimated age of 6.75 Ma (7.33 Ma, using Cande and Kent, 1992).

This event is recorded at Sites 844,845 , and 848 through 851 . Although present sample constraints are broad, this event at Site 844 correlates with an interval that represents the lower portion of paleomagnetic event $\mathrm{C} 3 \mathrm{Bn} . \mathrm{ln}$ to the upper portion of $\mathrm{C} 4 \mathrm{n}$. $\mathrm{ln}$ and has an age of between 6.89 and $7.32 \mathrm{Ma}$

21. LO Rossiella paleacea This event was used as a secondary marker species by Burckle (1978), by Barron (1985a, 1985b) for the eastern equatorial Pacific, and as a primary stratigraphic indicator by Schrader (1974) for the Indian Ocean. Burckle (1978) correlated this event to paleomagnetic event $\mathrm{C} 4 \mathrm{n} .1 \mathrm{n}$ and assigned it an age of $6.8 \mathrm{Ma}$ (7.40 Ma, using Cande and Kent, 1991). R. paleacea has occurred consistently throughout Sites 844,848 and 850 . Sample constraints for this event at Site 844 are similar to those for the FO of $N$. miocenica. Because of this, this event at Site 844 has an age constraint of 6.89 to 7.32 Ma.

22. FO Nitzschia reinholdii. $N$. reinholdii Kanaya ex Barron and Baldauf (1986) is a secondary stratigraphic marker that is useful in the low-latitude Pacific. Although this event can also be recognized in the North Atlantic and elsewhere, reliable calibration to a paleomagnetostratigraphy has been limited. Barron (1985a) assigned an age of 7.3 Ma (7.94 Ma, using Cande and Kent, 1992) for this event.
This event was observed at Sites 844 and 849 . At Site 844 , this event correlates with an interval that represents the lower portion of paleomagnetic event $\mathrm{C} 4 \mathrm{n}$. $\mathrm{In}$ to the upper portion of $\mathrm{C} 4 \mathrm{n} .2 \mathrm{n}$ and has an age older than $7.32 \mathrm{Ma}$. The lower age constraint cannot be directly correlated with the magnetostratigraphy at Site 844 .

\section{CONCLUSIONS}

Leg 138 provided a unique opportunity to examine the diatom assemblages from numerous near-continuous stratigraphic sequences from the eastern equatorial Pacific Ocean. Results of this study show that the standard diatom zonation of Barron (1985a, 1985b), for the most part, has been useful throughout this region. The somewhat inconsistent occurrence of Rhizosolenia praebergonii in Leg 138 samples required that the Rhizosolenia praebergonii and Nitzschia jouseae zones (as used by Barron, 1985a) be replaced by the Nitzschia marina and Nitzschia jouseae zones, as discussed by Baldauf (1984).

The near-continuous stratigraphic sequences and the excellent paleomagnetostratigraphy from several of the Leg 138 sites also allowed us to reevaluate previous age determinations for most of the Quaternary through upper Miocene diatom biostratigraphic events. In doing so, the age estimates determined here approximate those previously determined, once the previous ages were adjusted to Cande and Kent's chronology (1992).

\section{ACKNOWLEDGMENTS}

This manuscript was reviewed by Hans Schrader and John Barron. Funding for the research was supplied by Tohoku University and USSAC.

\section{REFERENCES*}

Akiba, F., and Yanagisawa, Y., 1986. Taxonomy, morphology and phylogeny of the Neogene diatom zonal marker species in the middle-to-high latitudes of the North Pacific. In Kagami, H., Karig, D.E., Coulbourn, W.T., et al., Init. Repts. DSDP, 87: Washington (U.S. Govt. Printing Office), 483-554.

Baldauf, J.G., 1984. Cenozoic diatom biostratigraphy and paleoceanography of the Rockall Plateau region, North Atlantic, Deep Sea Drilling Project Leg 81. In Roberts, D.G., Schnitker, D., et al., Init. Repts. DSDP, 81: Washington (U.S. Govt. Printing Office), 439-478.

1985. A high resolution late Miocene-Pliocene diatom biostratigraphy for the eastern equatorial Pacific. In Mayer, L., Theyer, F., Thomas, E., et al., Init. Repts. DSDP, 85: Washington (U.S. Govt. Printing Office), 457-475.

1987. Diatom biostratigraphy of the middle- and high-latitude north Atlantic Ocean, Deep Sea Drilling Project Leg 94. In Ruddiman, W.F., Kidd, R.B., Thomas, E., et al., Init. Repts. DSDP, 94 (Pt. 2): Washington (U.S. Govt. Printing Office), 729-762.

Baldauf, J.G., and Barron, J.A., 1991. Diatom biostratigraphy: Kerguelen Plateau and Prydz Bay regions of the Southem Ocean. In Barron, J., Larsen, B., et al., Proc. ODP, Sci. Results, 119: College Station, TX (Ocean Drilling Program), 547-598.

Baldauf, J.G., and Pokras, E.M., 1989. Diatom biostratigraphy of Leg 108 sediments: eastern tropical Atlantic Ocean. In Ruddiman, W., Sarnthein, M., et al., Proc. ODP, Sci. Results, 108: College Station, TX (Ocean Drilling Program), 23-34.

Barron, J.A., 1980. Upper Pliocene and Quaternary diatom biostratigraphy of Deep Sea Drilling Project Leg 54, tropical eastern Pacific. In Rosendahl, B.R., Hekinian, R., et al., Init. Repts. DSDP, 54: Washington (U.S. Govt. Printing Office), 455-485.

1981. Middle Miocene diatom biostratigraphy of DSDP Site 77B in the eastern equatorial Pacific. Geosci. J., 2:137-144.

, 1985a. Late Eocene to Holocene diatom biostratigraphy of the equatorial Pacific Ocean, Deep Sea Drilling Project Leg 85. In Mayer, L.,

\footnotetext{
Abbreviations for names of organizations and publication titles in ODP reference lists follow the style given in Chemical Abstracts Service Source Index (published by American Chemical Society).
} 
Theyer, F., Thomas, E., et al., Init. Repts. DSDP, 85: Washington (U.S. Govt. Printing Office), 413-456.

1985b. Miocene to Holocene planktic diatoms. In Bolli, H.M., Saunders, J.B., and Perch-Nielsen, K. (Eds.), Plankton Stratigraphy: Cambridge (Cambridge Univ. Press), 763-809.

Barron, J.A., and Baldauf, J.G., 1986. Diatom stratigraphy of the lower Pliocene part of the Sisquoc Formation, Harris Grade section, California. Micropaleontology, 32:357-371.

Barron, J.A., Keller, G., and Dunn, D.A., 1985. A multiple microfossil biochronology for the Miocene. In Kennett, J.P. (Ed.), The Miocene Ocean: Paleoceanography and Biogeography. Mem.-Geol. Soc. Am., 163:21-36.

Berggren, W.A., Kent. D.V., and Van Couvering, J.A., 1985. The Neogene: Part 2. Neogene geochronology and chronostratigraphy. In Snelling, N.J. (Ed.), The Chronology of the Geological Record. Geol. Soc. London Mem., 10:211-260.

Bodén, P., 1991. Reproductibility in the random settling method for quantitative diatom analysis. Micropaleontology, 37:313-319.

Bukry, D., and Foster, J.H., 1973. Silicoflagellate and diatom stratigraphy, Leg 16, Deep Sea Drilling Project. In van Andel, T.H., Heath, G.R., et al., Init. Repts. DSDP, 16: Washington (U.S. Govt. Printing Office), 815-871.

Burckle, L.H., 1972. Late Cenozoic planktonic diatom zones from the eastern equatorial Pacific. In Simonsen, R. (Ed.), First Symposium on Recent and Fossil Marine Diatoms. Nova Hedwegia Beih., 39:217-246

1977. Pliocene and Pleistocene diatom datum levels from the equatorial Pacific. Quat. Res. (N.Y.), 7:330-340.

1978. Early Miocene to Pliocene diatom datum level for the equatorial Pacific. Proc. Second Working Group Mtg. Biostratigraphic. Datum Planes Pacific Neogene, IGCP Proj. 114. Spec. Publ., Geol. Res. Dev. Ctr. 1:25-44.

Burckle, L.H., Keigwin, L.D., and Opdyke, N.D., 1982. Middle and late Miocene stable isotope stratigraphy: correlation to the paleomagnetic reversal record. Micropaleontology, 28:329-334.
Burckle, L.H., and Trainer, J., 1979. Middle and late Pliocene diatom datum levels from the central Pacific. Micropaleontology, 25:281-293.

Cande, S.C., and Kent, D.V., 1992. A new geomagnetic polarity time scale for the Late Cretaceous and Cenozoic. J. Geophys. Res., 97:13917-13951.

Harland, W.B., Cox, A.V., Llewellyn, P.G., Pickton, C.A.G., Smith, A.G., and Walters, R., 1982. A Geologic Time Scale: Cambridge (Cambridge Univ. Press).

Jousé, A.P., 1973. Diatoms in the Oligocene-Miocene biostratigraphic zones of the tropical areas of the Pacific Ocean. Nova Hedwigia Beih., 45:333364.

Koizumi, I., and Tanimura, Y., 1985. Neogene diatom biostratigraphy of the middle latitude western North Pacific, Deep Sea Drilling Project Leg 86. In Heath, G.R., Burckle, L.H., et al., Init. Repts. DSDP, 86 (U.S. Govt. Printing Office, Washington), 269-300.

Mayer, L., Pisias, N., Janecek, T., et al., 1992. Proc. ODP, Init. Repts., 138 (Pts. 1 and 2): College Station, TX (Ocean Drilling Program).

Mikkelsen, N., 1990. Cenozoic diatom biostratigraphy and paleoceanography of the western equatorial Indian Ocean. In Duncan, R.A., Backman, J., Peterson, L.C., et al., Proc. ODP, Sci. Results, 115: College Station, TX (Ocean Drilling Program), 411-432.

Sancetta, C., 1984. Diatoms from Leg 75, Deep Sea Drilling Project. In Hay, W.W., Sibuet, J.-C., et al., Init. Repts. DSDP, 75 (Pt. 2): Washington (U.S. Govt. Printing Office), 755-762.

Schrader, H.-J., 1974. Cenozoic marine planktonic diatom stratigraphy of the tropical Indian Ocean. In Fisher, R.L., Bunce, E.T., et al., Init. Repts. DSDP, 24: Washington (U.S. Govt. Printing Office), 887-967.

Date of initial receipt: 16 June 1993

Date of acceptance: 11 October 1993

Ms 138SR-107 